Rev. Biol. Trop., 47(4): 959-1006, 1999

www.ucr.ac.cr www.ots.ac.cr www.ots.duke.edu

\title{
El género Leptonema (Trichoptera: Hydropsychidae) en Costa Rica, con la descripción de una nueva especie
}

Fernando Muñoz-Quesada

Department of Entomology, University of Minnesota, 1980 Folwell Ave., St. Paul, MN 551 08, EUA, Correo electrónico munoz001@tc.umn.edu

Recibido 2-XII-1998. Corregido 13-IV-1999. Aceptado 16-IV-1999

\begin{abstract}
The genus Leptonema Guérin 1843 (Trichoptera: Hydropsychidae: Macronematinae) is one of the best known and most easily recognized of the Neotropical caddisflies. In the New World, the genus is widely distributed from southern North America through Central and South America, including the Antilles, but excluding souther Chile and adjacent Argentina. The adults of Leptonema are large (10-40 mm) with light brown to light green translucent wings. Some species have small black or dark spots or patterns on the forewings. Larvae of Leptonema build refuges with small silken nets, and this way feed by filtering fine organic particles from the water. The refuges and-nets are built with small gravel, sand, and silk. In 1914, Banks reported L. albovirens from Costa Rica, the first record of the genus for Costa Rica. In 1987, Flint $e t$ al. provided an exhaustive taxonomic review of the world species, and recognized 15 species groups, five of which were present in Costa $\mathrm{Ri}$ ca. In addition, they described 48 new species, four from Costa Rica, and reported five additional records. Holzenthal added six additional species records, and Muñoz-Q. described five new species, of these $L$. tapanti is the first species in the stigmosum Group defined by Flint et al. and present in the country. In total, 24 species of Leptonema have been previously recorded in Costa Rica. For this research, collections of Leptonema specimens in the University of Minnesota Insect Collection, St. Paul, MN and the National Museum of Natural History, Smithsonian Institution, Washington, DC. were examined. In this paper, one additional undescribed species from Costa Rica is diagnosed, described and illustrated: Leptonema tica. Diagnoses and illustrations of the male genitalia of the other species are presented. Figures of the larva and adult, as well as aspects of natural history of the genus are included. Finally, an identification key in English and Spanish is provided, together with distribution record maps of all species in Costa Rica are included.
\end{abstract}

Key words: Leptonema, Trichoptera, caddisfly, aquatic insects, new species, life stages, key, Costa Rica, Neotropics.

El género Leptonema Guérin 1843 (Hydropsychidae: Macronematinae) es uno de los géneros de Trichoptera más abundantes y más ampliamente distribuidos en Latinoamérica. Las especies de este género se distribuyen principalmente en el Nuevo Mundo, desde el sur de EUA, hasta el norte de Chile y centro de Argentina, incluyendo las Antillas, sumando 99 especies (2 especies son consideradas $\mathrm{No}$ mina dubia, Flint et al. 1987, Flint 1991, Muñoz-Q. 1997), mientras que 10 son conocidas en África y 6 en Madagascar (Flint et al. 1987). En Costa Rica, Leptonema es uno de los géneros más comúnmente recolectados y más ampliamente distribuidos dentro del orden Trichoptera. Los individuos adultos de este género son grandes $(10-40 \mathrm{~mm})$, con alas translúcidas de color verde claro a pardo claro; las alas anteriores de muchas especies presentan patrones de pelillos oscuros (Flint et al. 1987).

El género fue establecido por Guérin en 1843 al describir L. pallidum de Brasil. En 1914, Banks reportó el primer registro de distribución de una especie de este género en Costa Rica, L. albovirens (Walker). Mosely (1933) en su revisión del género comunicó dos registros 
de distribución más para el país. Posteriormente, Flint et al. (1987) realizaron una revisión taxonómica completa de la fauna mundial del género, donde además, trataron aspectos biogeográficos y filogenéticos. En dicha revisión establecieron 15 grupos de especies, de los cuales, sólo cinco tenían especies presentes en Costa Rica, y además describieron 48 nuevas especies, cuatro de las cuales fueron para este país, lo mismo que cinco nuevos registros de distribución. Holzenthal (1988) agregó seis nuevos registros de distribución para esta región. Finalmente, MuñozQ. (1997) describió cinco nuevas especies, entre ellas $L$. tapanti, la primera especie del Grupo stigmosum en Costa Rica, e incluyó los primeros registros de distribución para $L$. cheesmanae Mosely en Costa Rica. En el proceso de revisión de especímenes para esta investigación se encontró una especie no descrita perteneciente al Grupo simulans. En total, en Costa Rica son conocidas actualmente 25 especies, distribuidas en seis de los grupos de especies definidos por Flint et al. (1987) (Cuadro 1).

En el presente estudio se ofrece la descripción de esta especie no descrita, así como las descripciones diagnósticas de las otras 24 especies conocidas del género Leptonema para Costa Rica, basándose estas descripciones en los genitales de los machos, específicamente en el phallus. Además, se ilustra el phallus de cada una de las especies y se presenta una clave en español e inglés para la identificación de todas estas especies conocidas. Se provee la ilustración de la larva y del adulto de Leptonema, y anotaciones sobre aspectos de la historia natural del género en Costa Rica. Se incluyen mapas con los datos de la distribución conocida de estas especies.

Este trabajo presenta parte de los resultados del Proyecto "Inventario de los Trichoptera de Costa Rica" apoyado por la Colección de Insectos de la Universidad de Minnesota ("Insect Collection", UMSP), St. Paul, MN, EUA, la "U.S. National Science Foundation" (USNSF), Washington, DC,
EUA, y en la etapa inicial, con el apoyo del Instituto Nacional de Biodiversidad (INBio), Costa Rica, para recolectar, describir y catalogar la fauna de los tricópteros de Costa Rica.

\section{MATERIALES Y MÉTODOS}

Los adultos se recolectaron de dos maneras. La primera, y la más general, consiste en utilizar luces ultravioleta y/o luces blancas suspendidas frente a una pantalla o sábana blanca. Este equipo es colocado a un lado del río o quebrada, preferiblemente con las luces de frente al cauce del agua y encendidas desde las 18: 00 (o al atardecer) hasta las 22: 00 horas aproximadamente; los tricópteros son así atraídos por la luz a la pantalla, donde son recolectados con frascos de cianuro. Posteriormente, los especímenes son montados en alfileres entomológicos y guardados en una caja hermética. En el laboratorio, se separan las hembras de los machos, con la ayuda de un microscopio de disección (estereoscopio). Los tricópteros machos son colocados en una cámara húmeda para que se flexibilizen. Una vez flexibles los especímenes, se les separa el abdomen (se puede cortar preferiblemente desde la base del abdomen o desde el segundo segmento), el cual contiene los genitales para ser aclarados. Para tal fin, los genitales son introducidos en $\mathrm{KOH}$ al $10 \%$ (aproximadamente de 5 a 10 minutos en $\mathrm{KOH}$ al $10 \%$ caliente, de 4 a 6 horas en $\mathrm{KOH}$ al $10 \%$ a temperatura ambiente), se colocan en agua para limpiarlos internamente con la ayuda de una jeringa de punta muy fina bajo el estereoscopio, se colocan posteriormente en alcohol ácido por un minuto, después en alcohol etílico al $80 \%$ por otro minuto, y por último, en glicerina para su observación e identificación. Para su depósito final, se guardan en un microvial con glicerina junto al resto del cuerpo del tricóptero (de preferencia en el mismo alfiler). 


\section{CUADRO 1}

Lista de las especies y grupos de especies de Leptonema Guérin, establecidos por Flint et al. (1987), reportados para Costa Rica, de acuerdo a los registros de distribución publicados por Flint et al. (1987), Holzenthal (1988), Aguila S. (1992) y Muñoz-Q. (1997).

Especies

Grupo crassum

L. crassum Ulmer 1905

L. divaricatum Flint, McAlpine, Ross 1987

Grupo stigmosum

L. tapanti Muñoz-Q. 1997

Grupo plicatum

L. ekisi Flint, McAlpine, Ross 1987

L. flintorum Muñoz-Q. 1997

L. fortunum Flint, McAlpine, Ross 1987

L. hamuli Flint, McAlpine, Ross 1987

L. huismanae Muñoz-Q. 1997

L. rafita Muñoz-Q. 1997

L. salvini Mosely 1933

L. sinuatum Mosely 1933

L. turrialbum Flint, McAlpine, Ross 1987

L. vitum Flint, McAlpine, Ross 1987

L. woldianum Flint, McAlpine, Ross 1987

Grupo simulans

L. asclepium Flint, McAlpine, Ross 1987

L. campanum Flint, McAlpine, Ross 1987

L. simulans simulans Flint, McAlpine, Ross 1987

L. tica, nueva especie

Especies

Grupo pallidum

L. albovirens (Walker) 1852

Grupo complexum

\section{L. cheesmanae Mosely 1933}

L. clorito, Muñoz-Q. 1997

L. complexum Mosely 1933

L. forficulum Mosely 1933

L. furciligerum Flint, McAlpine, Ross 1987

L. intermedium Mosely 1933
Distribución

México, Guatemala; Honduras; Nicaragua; Costa Rica: Ala juela, Guanacaste, Heredia, Limón, Puntarenas, San José; Panamá; Colombia; Venezuela; Bras il; Perú; Paraguay; ARgENTINA.

Costa Rica: Alajuela, Heredia, Limón; Colombia; VENEZUELA; ECUADOR.

Costa Rica: Cartago; Panamá.

Costa Rica: Ala juela, Cartago; Panamá.

Costa Rica: Puntarenas.

Costa Rica: Cartago; Panamá.

Costa RicA: Cartago, Guanacaste, Limón; Panamá.

Costa Rica: Alajuela, Guanacaste.

Costa Rica: Alajuela, Cartago, San José.

Costa Rica: Ala juela, Cartago, Puntarenas, San José; Panamá. Costa Rica: Puntarenas, San José; Panamá; Colombia.

Costa Rica: Alajuela, Cartago, Heredia, Limón.

Costa Rica: Puntarenas.

Costa Rica: Ala juela, Cartago, Guanacaste; PanamÁ.

Costa Rica: Ala juela, Cartago, Guanacaste, Limón, San José Costa Rica: Alajuela, Puntarenas, San José; Panamá.

Costa RicA: Alajuela, Cartago, Guanacaste, Puntarenas,

San José; Panamá.

Costa Rica: San José

Distribución

E.U.A.: Texas; MÉXICo; Belice; Guatemala; Honduras; Nicaragua; Costa Rica: Alajuela, Cartago, Guanacaste, Heredia, Limón, Puntarenas, San José; Panamá; Colombia; Venezuela; Trinidad \& Tobago; Granada; San Vicente.

Costa RicA: Ala juela, Guanacaste, Limón, San José;

Panamá; Colombia.

Costa Rica: Ala juela.

Costa RicA: Alajuela, Cartago, Guanacaste, Heredia, Limón, Puntarenas, San José; Panamá.

Costa Rica: Alajuela, Guanacaste; Panamé.

COSTA RicA: Puntarenas.

Costa Rica: Alajuela, Cartago, Guanacaste, Heredia, Limón, Puntarenas, San José; Panamá; Colombia; Ecuador. 
Los adultos también pueden ser recolectados con trampas de alcohol. Dichas trampas consisten de un recipiente amplio de 5 a $10 \mathrm{~cm}$ de profundidad, con el fondo de color blanco y un cedazo en la parte superior (de 1 $\mathrm{cm}^{2}$ de malla, con este tamaño de abertura se evita la caída en el fondo de la trampa de coleópteros, lepidópteros y otros individuos grandes que dañarían a los tricópteros). Un $70 \%$ del recipiente se llena con alcohol etílico al $70 \%$ y sobre el cedazo se coloca un tubo de luz fluorescente ultravioleta o/y blanca. Todo el equipo se coloca a unos metros al lado del cauce del agua, de las 18: 00 horas (o al atardecer) hasta las 06: 00 horas del otro día. Inmediatamente después, el material recolectado se deposita en alcohol etílico al 90\%. En el laboratorio se obtienen los tricópteros machos para aclarar los genitales e identificarlos siguiendo el mismo procedimiento mencionado anteriormente, solamente que los genitales se depositan finalmente en un microvial con alcohol etílico al $80 \%$ junto al resto del cuerpo.

Esta revisión se fundamentó estrictamente en la morfología del phallus para identificar y diagnosticar las especies de Leptonema conocidas a la fecha en Costa Rica. Se sigue la terminología propuesta por Flint et al. (1987) para las estructuras del órgano genital masculino; la Fig. 3 muestra las vistas dorsal, lateral y ventral de un phallus hipotético con las respectivas estructuras o procesos rotulados. Para los demás términos morfológicos mencionados de los adultos, se consultó la terminología utilizada por Ross (1982), Torre-Bueno (1989), Neboiss y Dean (1991), Angrisano (1995) y Wiggins (1996b). A pesar de que los caracteres diagnósticos están basados en la morfología del phallus, se of recen breves descripciones del patrón de coloración de la ala anterior siguiendo el diagrama que se presenta en la Fig. 2 A. Por concordancia etimológica con el nombre del orden Trichoptera, cuyo significado es: "ala con pelos" (del griego, "trichos"= pelos, "pteron"= ala), se utilizan los términos "pelos" o "pelillos" de las alas, de las patas, etc., de las larvas y adul- tos, en el entendido de que estas estructuras son morfológicamente "sedas" o "setas" (inglés: "seta", plural: "setae"; latín: "seta", "saeta"; Williams y Wiggins 1981, TorreBueno 1989, Snodgrass 1993; Ross 1982, fig. 100 , utiliza "seda", sin embargo otros autores usan "seta": Quintanilla y Fraga 1969, Greiff 1985, Correa G. 1988, Angrisano 1995). El término "branquia" aquí corresponde a los términos morfológicos en inglés "gill" y "traqueal gill" (Torre-Bueno 1989, Morse y Holzenthal 1996, Wiggins 1996a, 1996b), como es usado por Ross 1982, Angrisano 1995, sin embargo otros autores en español usan los términos "agalla branquial" (Correa G. 1988), o "traqueobranquia". Una branquia doble corresponde a dos branquias simples que comparten una misma base (Scott 1983, fig. 168). Terminología morfológica de los estados inmaduros de tricópteros puede ser encontrada en McCafferty (1983), Correa G. (1988), Neboiss y Dean (1991), Angrisano (1995), Morse y Holzenthal (1996) y Wiggins (1996a, 1996b). Se utiliza el término en latín cuando no existe el correspondiente en español, tales son los casos de "cord", por el cual se entiende el área que va sobre las venas transversales desde la " $r_{3}-r_{4}$ " hasta la " $m-c u$ " de la ala anterior; "nygma" (plural: "nygmata") punto sensorial localizado basalmente entre las venas " $R_{4}$ y $R_{5}$ " en las alas anterior y posterior, y apicalmente en la celda tiridial $(c t h)$ de la ala anterior (Fig. 2).

El material examinado fue recolectado principalmente por Holzenthal y sus colegas durante 1986 a 1992 y está depositado mayormente en la Colección de Insectos de la Universidad de Minnesota ("Insect Collection", UMSP) St. Paul, MN, EUA, en caso contrario, se indicará las siglas de la institución donde el material está depositado. Las siglas utilizadas de las Intituciones o Museos que custodian los holotipos y demás material examinado corresponden a: BMNH, British Museum of Natural History (Londres, Inglaterra); DEI, Institut für Pflanzenschutzforschung der Akademie der Landwirtswissenschaften, Abteilung der Inserkten, Eberswalde (Alemania); INBio, Insti- 
tuto Nacional de Biodiversidad (Costa Rica); MCZ, Museum of Comparative Zoology, Harvard University, Cambridge (Massachussets, E.U.A.); MNHNP, Muséum National d'Historie Naturelle, Paris (Francia); NMNH, National Museum of Natural History, Smithsonian Institution (Washington, DC, E.U.A.; anteriomente abreviado. USNM, United States National Museum); UMSP, Insect Collection of the University of Minnesota, St. Paul (Minnesota, E.U.A.); ZSZMH, Zoologisches Staatsinstitut und Zoologisches Museum, Hamburg (Alemania).

El esquema de presentación de las especies en el presente trabajo es el siguiente: las especies se agruparon alfabéticamente dentro de su grupo de especies correspondiente, y los grupos de especies se ordenaron de acuerdo al orden filogenético presentado por Flint et al. (1987), como se muestra en el Cuadro 1.

Posterior a la descripción en inglés de $L$. tica, nueva especie, se adjunta su respectiva descripción diagnóstica en español. Finalmente, se presenta una clave para la identificación de los machos de las especies conocidas de Leptonema en Costa Rica basada en el phallus, dicha clave está en español e inglés.

\section{Leptonema Guérin}

Leptonema Guérin 1843: 165, 396; Ulmer 1907a: 44-61 ; 1907b: 162-163; 1957: 346-348, 352, 354; Mosely 1933: 1-69; Fischer 1947: 313-315: 1963: 165-174; 1972: 156,157; Marlier 1962: 133-135; Scott 1983: 389-398; Flint, McAlpine y Ross 1987: 1-193. (Especie tipo Leptonema pallida Guérin, monobásica).

Neoleptonema Ulmer 1907a: 61-62 (Especie tipo, Neoleptonema aspersum Ulmer, monobásica); Flint 1974a: 103; Flint, McAlpine y Ross 1987: 1-193 (sinonimia).

Diagnosis.-Adultos (Fig. 1; Flint et al. 1987, figs. 1-8): Longitud de $10 \mathrm{~mm}$ a $40 \mathrm{~mm}$. Cabeza y sus apéndices ligeramente cubiertos dorsalmente con pelillos pardos claros; antenas largas, usualmente más largas que las alas anteriores; escapo corto y grueso; flagelo delgado; ocelos ausentes; palpos maxilares con cinco segmentos, el segmento apical $\left(5^{\text {to }}\right)$ el más largo; palpos labiales con tres segmentos. Tórax cubierto ligeramente con pelillos pardos (densamente en algunas especies, como L. divaricatum y L. crassum, Fig. $1 \mathrm{C}$ ); patas largas y fuertes; fórmula de las espinas tibiales: 1-4-4 o 2-2-4 (esta última para las especies conocidas en Costa Rica); alas translúcidas de color verde claro a pardo cubiertas ligeramente con pelillos pardos y con "nygmata" presentes; alas anteriores a veces con variados patrones pequeños de pelillos más oscuros y con ramificaciones apicales 1, 2, 3, 4 y 5, celdas discoidal $(\mathrm{cd})$, medial $(\mathrm{cm})$ y tiridial $(\mathrm{cth})$ presentes (Fig. 2 A); alas posteriores más anchas, pequeñas y pálidas que las anteriores, y con ramificaciones apicales 1, 2, 3 y 5 (Fig. 2 B).

Macho: Aparato genital (Fig. 23) en vista lateral, con el segmento abdominal IX generalmente alargado dorsoventralmente, en vista dorsal, generalmente con una proyección superficial y semejante a una "V" invertida (Fig. 23 B). Segmento abdominal $\mathrm{X}$ de aspecto membranoso y generalmente con varios elementos, en vista lateral, generalmente triangular (Fig. 23 A). Apéndice inferior ("clasper") bisegmentado, largo, tubular y con setas pequeñas principalmente en el margen interno del segmento apical, este último generalmente más pequeño que el basal (Fig. 23 C). Phallus de aspecto tubular, delgado y alargado, la sección apical con la mayoría de los procesos " $a$ j" (Fig. 3; Flint et al. 1987, figs. 9-12).

Hembra: Aparato genital (Flint et al. 1987, fig. 13) con el segmento abdominal VIII dividido ventralmente en dos placas o valvas con setas pequeñas. Segmento abdominal IX (tergo) simple, subtriangular y dirigido posteriormente, con filas de setas en cada lado posterodorsalmente, a cada lado posteroventralmente con un receptáculo y un surco para el acople del apéndice inferior ("clasper") del macho. Segmento abdominal X (tergo) pequeño, angosto y apicolateralmente, con cercos y un par de papilas.

Pupa: Placas dorsoabdominales con procesos esclerotizados pequeños en forma de ganchos o garfios, distribuidas anteriormente en segmentos II o III-VIII, y posteriormente en 
el segmento III (Flint y Wallace 1980; Flint, et al., 1987). Descripciones e ilustraciones de pupas de especies de Leptonema son encontradas en Roback (1996) Flint y Wallace (1980), Flint et al. (1987) y Scott (1983).

Diagnosis: Pupas de Calosopsyche (Flint y Bueno-Soria 1987) y Smicridea (Flint 1964, 1968, 1974b, 1989), son similares a la de Leptonema, sin embargo difieren en la distribución de las placas dorsoabdominales con procesos esclerosados. En Calosopsyche y Smicridea, la distribución de las placas dorsoabdominales es la siguiente: anteriormente en segmentos IIVIII, y posteriormente en segmentos III-IV.

Larva (Fig. 4): CuERPO robusto, redondeado y cubierto densamente por setas de tamaño regular, oscuras, delgadas y tubulares, semejantes a un pelo; en general, las partes esclerosadas de color pardo oscuro. CABEZA (Fig. 4 A, B, C) esclerotizada, alargada y convexa ligeramente; en vista dorsal, suturas clípeofrontal y coronal tenues, esta última curvada levemente; apotema clípeofrontal triangular, alargado y puntiagudo posteriormente, el margen anterior aserrado ligeramente; en vista ventral (Fig. 4 C), el margen anterior escotado irregularmente, y el margen posterior redondeado con una hendidura mesial; sutura ecdisial tenue y dividiendo totalmente la gena; submentón esclerosado, subtriangular y cóncavo anteromesalmente; apotema anteroventral esclerosado, triangular y conspicuo; apotema posteroventral esclerotizado, triangular y diminuto o inconspicuo. Antena no distinguible; labrum semiovalado; mandíbulas esclerosadas fuertemente y asimétricas. Tórax (Fig. 4 A, B). Protórax con el pronoto esclerotizado, éste último, con los margénes posterolateral oscuro y el anterior con pelos pequeños; pata protorácica (Fig. 4 E, F) más pequeña, robusta y oscura que las otras patas; trocantín esclerosado, puntiagudo, no bifurcado y con setas; coxa esclerotizada con setas mesodorsales y sin ningún lóbulo mesodistal; fémur y tibia con pelos finos y principalmente esparcidos mesialmente; fémur esclerotizado, alargado y rectangular, el margen dorsomesial con un proceso raspador (Fig. 4 F); tibia y tarso esclerosados; uña tarsal esclerosada, curvada y puntiaguda; protórax en vista dorsal (Fig. 4 B), subcuadrado ligeramente, con el margen posterior cóncavo ligeramente y con un par de setas posteromesales largas; línea ecdisio-mediodorsal tenue; en vista ventral (Fig. $4 \mathrm{D}$ ), proesternito con una placa proesternal esclerosada, rectangular y seguida de un esclerito semiovalado; branquias ausentes. Mesotórax con el mesonoto esclerosado, éste último, con los margénes posterolateral oscuro y el anterior con pelos pequeños; pata mesotorácica similar en forma y tamaño a la pata metatorácica, delgadas, alargadas y con pelos finos mesialmente; mesotórax en vista dorsal, subcuadrado, con una proyección posteromesal y con setas largas posteriormente; en vista ventral, con una branquia (ventrolateral) simple en la base de cada pata. Metatórax con el metanoto esclerotizado, éste último, con los margénes posterolateral oscuro y el anterior con pelos pequeños; metatórax en vista dorsal, subcuadrado, con el margen posterior cóncavo levemente y con setas largas posteriormente; en vista ventral, con una branquia (ventrolateral) simple en la base de cada pata y un par de branquias ventrales simples anteromesialmente. ABDomen (Fig. 4 A, G, H) robusto, tubular y largo, hasta dos veces la longitud de la cabeza y el tórax juntos, cubierto dorsolateralmente de setas delgadas, tubulares y de tamaño regular, semejantes a un pelo; ventral y ventrolateralmente con branquias simples y dobles, las branquias con muchos filamentos branquiales distribuidos uniformente a todo lo largo del tallo de la branquia (una branquia doble consiste de dos branquias simples que comparten una misma base, Fig. 4 A; Scott 1983, fig. 168). La distribución de las branquias abdominales en vista ventral, es como sigue: segmento I, con una branquia ventrolateral simple a cada lado y un par de branquias ventrales simples anteromesialmente; segmentos II-VI, con una branquia ventrolateral doble a cada lado y un par de branquias ventrales simples anteromesialmente; segmento VII, con una branquia ventrolateral doble a cada lado. Segmentos abdominales III-VI o VII, presentan un proceso lateral alargado de pelos pardos, semejante a 
un penacho o fleco. Segmento VIII (Fig. $4 \mathrm{H}$ ) con un par de escleritos mesoventrales pequeños y triangulares con setas posteriores largas. Segmento IX (Fig. $4 \mathrm{G}, \mathrm{H}$ ) con un penacho posterolateral de setas largas, y un par de escleritos mesoventrales conspicuos y triangulares con setas largas en el margen posterior. Pata anal (Fig. 4 G, H) con un esclerito laterobasal curvado, un apéndice anal largo y esclerosado dorsalmente con setas largas y dorsoapicales principalmente, y una uña anal esclerotizada, robusta, curvada y puntiaguda.

Material examinado: Costa RICA: San José: Parque Nacional Braulio Carrillo, 6.2 km $\mathrm{NE}$ de Oficinas Administrativas, $10.09^{\circ} \mathrm{N}$, $83.97^{\circ} \mathrm{W}$, el. $1100 \mathrm{~m}, 6 . i \mathrm{i} .1986$, Holzenthal y Morse, 13 larvas (en alcohol) (UMSP).

Descripciones e ilustraciones de larvas de especies de Leptonema son encontradas en Flint $(1964,1968)$, Roback (1966), Flint y Wallace (1980), Flint et al. (1987), Correa $e t$ al. (1981), Correa G. (1988), Scott (1983), Angrisano (1995), Morse y Holzenthal (1996) y Wiggins (1996a).

Diagnosis: Larvas de Calosopsyche (Flint y Bueno-Soria 1987) y Smicridea (Flint 1974b, 1989, Correa G. 1988, Angrisano 1995, Morse y Holzenthal 1996, Wiggins 1996a), son similares a las de Leptonema, sin embargo difieren en la forma de las setas que cubren el cuerpo, en la forma y distribución de las branquias, y en la forma de los procesos laterales de los segmentos abdominales III-VI o VII.

Las setas que cubren el cuerpo, y principalmente el abdomen en Leptonema y Calosopsyche, son similares, de tamaño regular, oscuras, tubulares y en forma de pelo (Morse y Holzenthal 1996, figs. 18.68-69, "plain hair"); y en Smicridea, son de tamaño regular, aplanadas, más anchas apicalmente que basalmente, en forma de escama (Morse y Holzenthal 1996, fig. 18.68, "scale hair"; Wiggins 1996a, fig. 7.10 A).

Las branquias en Leptonema y Calosopsyche, son similares en la aparencia, con muchos filamentos branquiales distribuidos uniformente a todo lo largo del tallo de la branquia (Fig. 4 A; Correa G. 1988, fig. 119 c; Morse y Holzenthal 1996, fig. 18.47; Wiggins 1996a, fig.
7.6 A); y en Smicridea, éstas tienen pocos filamentos branquiales localizados y distribuidos irregularmente, principalmente en la mitad apical del tallo de la branquia (Correa G. 1988, fig. $120 \mathrm{c}$; Morse y Holzenthal 1996, fig. 18.48; Wiggins 1996a, fig. 7.10 A)

La distribución de las branquias es diferente en Leptonema y Calosopsyche, el metatórax presenta en Leptonema, un par de branquias ventrolaterales simples y un par de branquias ventrales simples, mientras que en Calosopsyche, presenta un par de branquias ventrolaterales dobles y un par de branquias ventrales simples; el segmento abdominal I presenta en Leptonema, un par de branquias ventrolaterales simples y un par de branquias ventrales simples, en tanto que en $\mathrm{Ca}$ losopsyche, solamente un par de branquias simples; el segmento abdominal VII presenta en Leptonema, una branquia ventrolateral doble a cada lado, mientras que en Calosopsyche, están ausentes (Scott 1983, Flint y Wallace 1980, Flint y Bueno 1987, Flint et al. 1987). Las distribuciónes de las branquias en Leptonema y Smicridea, son similares, sin embargo difieren en que el metatórax presenta en Leptonema, dos pares de branquias, y en Smicridea, pueden ser uno o dos pares de branquias (Flint 1974b, 1989, Flint y Wallace 1980, Flint et al. 1987, Scott 1983).

En Leptonema, los segmentos abdominales . III-VI o VII presentan un proceso lateral alargado de pelos pardos semejante a un penacho o fleco en cada segmento (Fig. 4 A; Correa G. 1988, figs. 119 a; Wiggins 1996a, fig. 7.6 A); en Calosopsyche, los segmentos abdominales IIIVII presentan de uno a tres pequeños procesos membranosos laterales en cada segmento (Flint y Bueno 1987, fig. 7); y en Smicridea, los segmentos abdominales III-VII presentan de uno a tres procesos filamentosos laterales en cada segmento (fig: 1 en Flint 1974b, 1989).

Finalmente, en la pata anal, el penacho de pelos o setas largas es mucho más abundante y desarrollado en Calosopsyche (Flint y Bueno 1987, fig. 7) y Smicridea (fig. 1 en Flint 1974b, 1989; Wiggins 1996a, fig. 7.10 A), que en Leptonema (Fig. 4 G; Wiggins 1996, fig. 7.6 A).

La Fig. 120 de Correa G. (1988) corresponde al subgénero $S$. (Rhyacophylax) que 

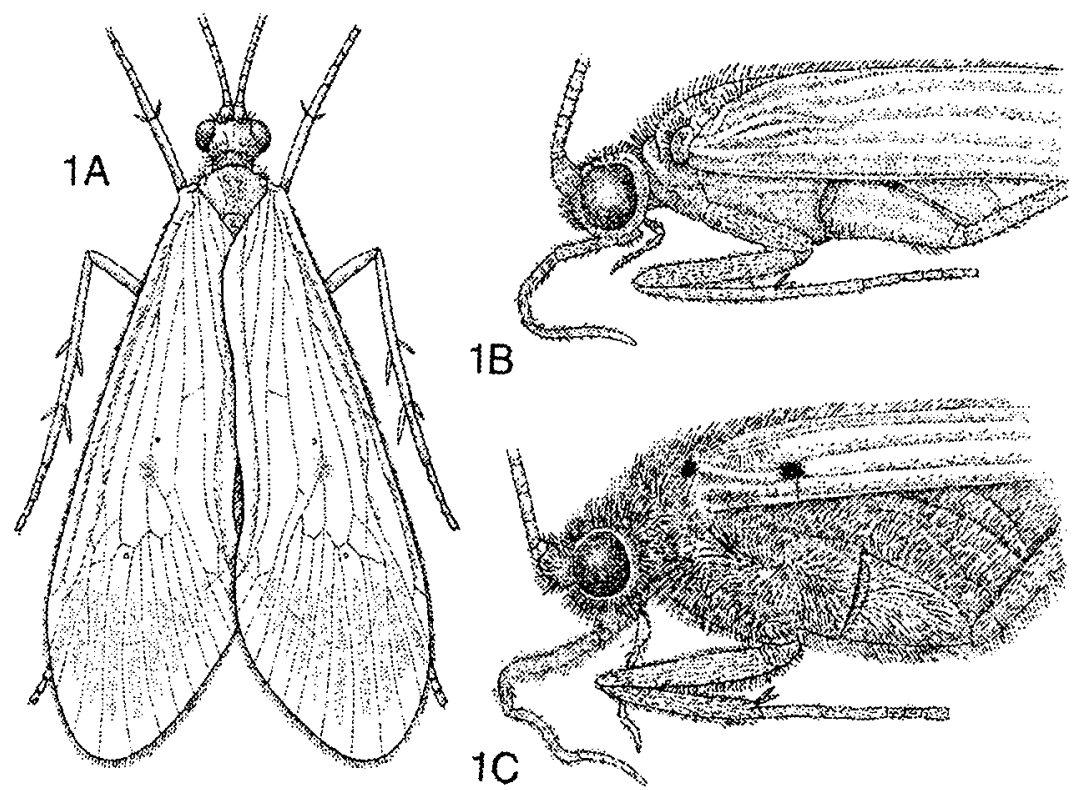

Fig. 1. Adultos.-A., L. complexum., vista dorsal; B., vista anterolateral; C., L. crassum, vista anterolateral.

presenta dos escleritos mesoventrales en el segmento abdominal VIII, en tanto que el subgénero $S$. (Smicridea), presenta un esclerito mesoventral en el segmento abdominal VIII (Wiggins 1996a, fig. 7.10 E).

Biología: Las larvas y adultos de Leptone$m a$ habitan durante todo el año en bosques de 15 a $2000 \mathrm{~m}$ de altura con vegetación arbórea abundante y masas de aguas poco contaminadas, aunque L. albovirens ha mostrado ser tolerante a niveles considerables de contaminación causada por aguas residuales domésticas y por carga orgánica residual de las poblaciones aledañas (obs. pers.). Las larvas de Leptonema son encontradas usualmente en ríos, quebradas, acequias, riachuelos con aguas de poca corriente a corriente constante, con arena, piedras y rocas en el lecho, y con hojas, palos y troncos entre las piedras y rocas. Las larvas construyen los refugios o casas con arena, fragmentos de material orgánico y minerales, los cuales los adhieren fuertemente con seda que ellas producen a las superficies de las piedras, rocas, y palos o troncos sumergidos. Asimismo, junto a los refugios, las larvas construyen con seda pequeñas redes para atrapar alimento (invertebrados pequeños, algas, material vegetal). Las larvas al alcanzar su madurez construyen un pupario con seda, fragmentos de material orgánico y minerales, el cual adhieren fuertemente y usualmente debajo de rocas o piedras. Los adultos emergen a la superficie y permanecen en la vegetación para copular. Las hembras depositan los huevos fertilizados en la superficie del agua, aunque algunas especies se sumergen y deposi$\tan$ o adhieren los huevos en el fondo o a objetos sumergidos, para lo cual presentan tibias aplanadas y acanaladas en la superficie interna como remos, y el cuerpo densamente cubierto de pelos que les permiten nadar dentro del agua, como en $L$. crassum (Fig. 1C). Los adultos son usualmente recolectados al atardecer y en las primeras horas de la noche cuando son más activos y vuelan hacia las trampas de luz (ultravioleta o/y blanca) atraídos por la luz.

\section{Leptonema crassum Ulmer}

Figs. 1 C, 5; Mapa 4

Leptonema crassum Ulmer 1905: 58-59, Pl. II, figs. 52-53, ơ, Brasil: Espíritu Santo (ZSZMH, holotipo ơ destruído en 1943); Mosely 1933: 12-13, figs. 1-3; Fischer 1963: 168; 1972: 156; Weidner 1964: 84; Flint 1966: 5; 

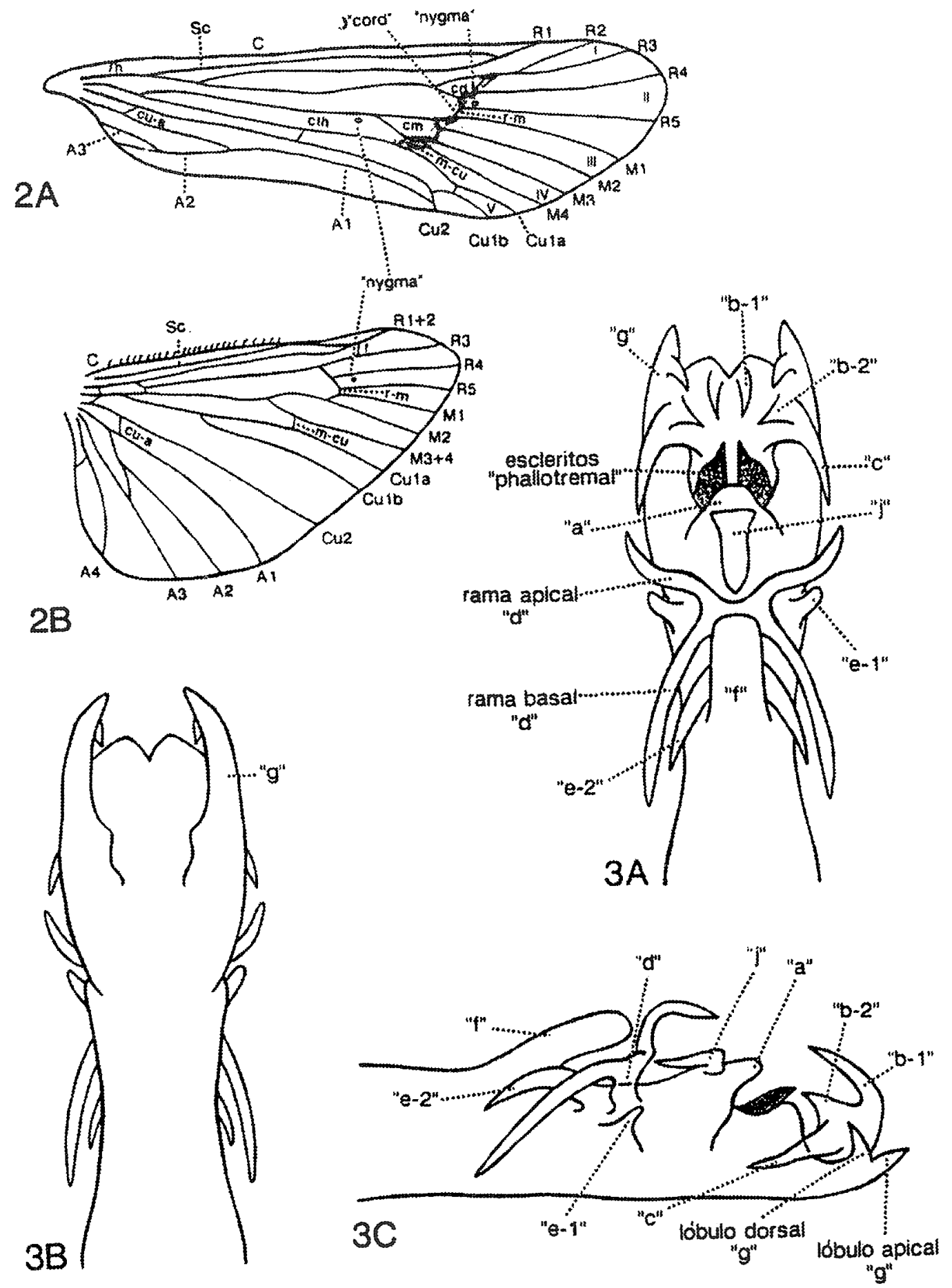

Fig. 2-3. 2: Alas de L. complexum. - A., ala anterior; B., ala posterior.

3: Phallus hipotético de un macho de Leptonema con procesos "a", "b", "c", "d", "e", "f", "g" y "j".- A., vista dorsal; B., vista ventral; C., vista lateral. 


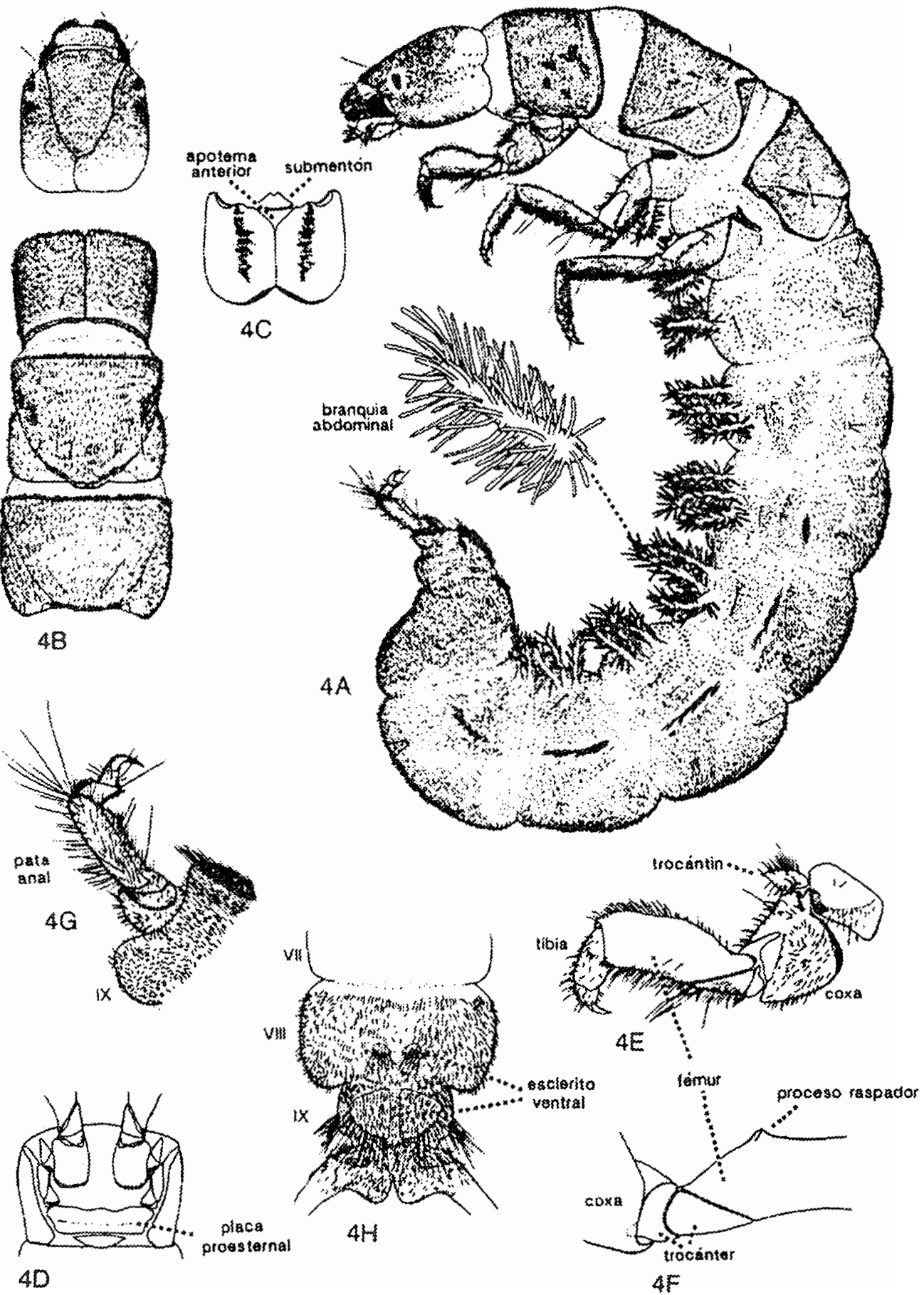

Fig. 4. Larva de Leptonema sp.- A., larva, vista lateal; B., cabeza y tórax, visa dorsal; C., cabeza, vista ventral; D., protórax, vista ventral; E., pata protorácica, vista lateral; F., paa protorácica, vista internolateral; G., segmento abdominal IX y pata anal, vista lateral; H., segmentos abdominales VIII y IX, vista ventral. 

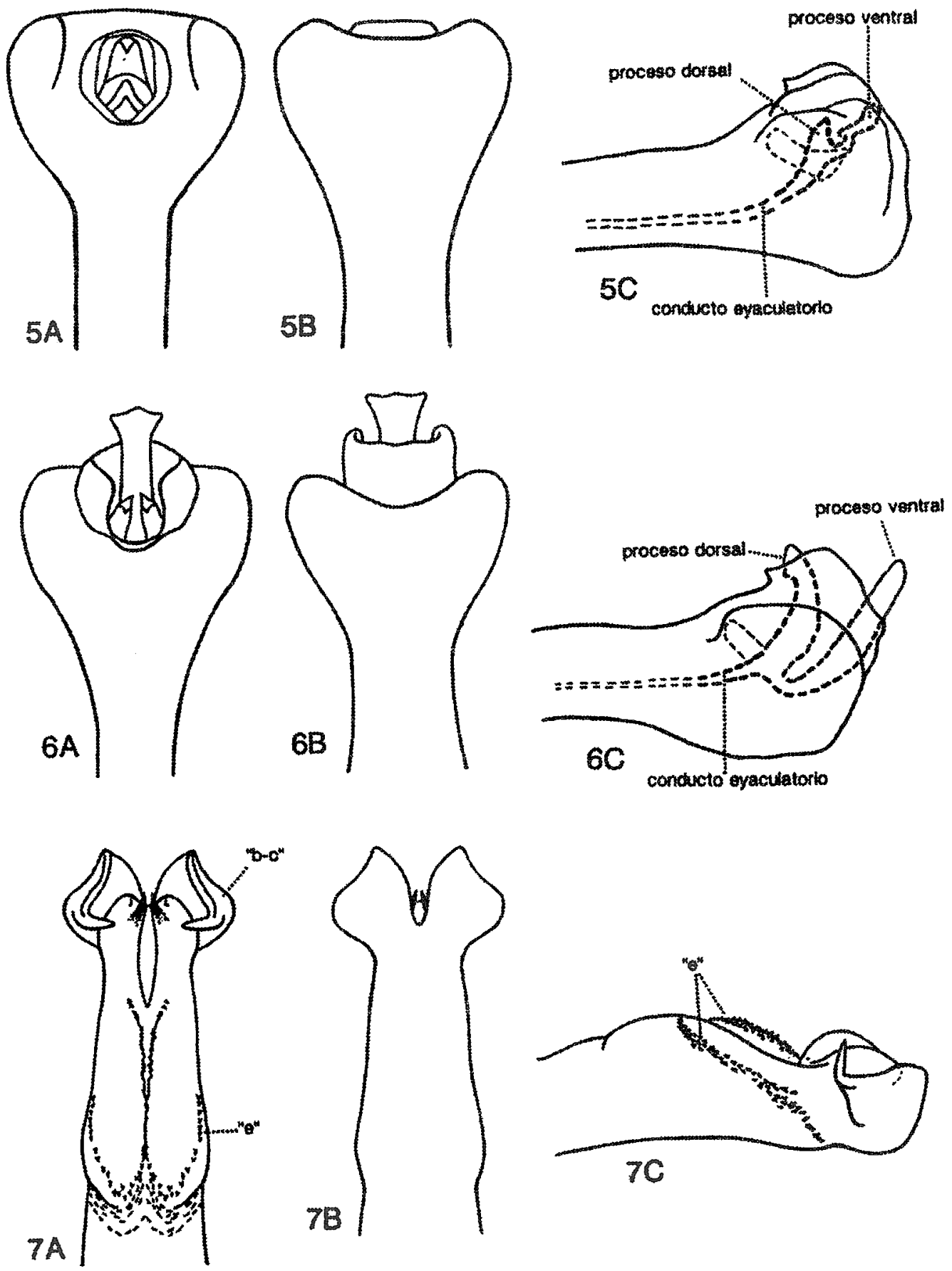

Figs. 5/7. Phallus. 5. L. crassum, 6. L. divaricatum, 7. L. tapanti. -A., vista dorsal; B., vista ventral; C., vista lateral. 
1972: 235 [Argentina]; 1978: 385; Bueno-Soria y Flint 1978: 209; Flint, McAlpine y Ross 1987: 34-36, figs. 226-233; Holzenthal 1988: 66.

Leptonema radiale Navás 1927: 42, \%, Brasil: Minas Gerais (DEI); Flint 1978: 385 (sinonimia).

Leptonema grisolinum Navás 1933a: 312313, fig. 23, ơ, Venezuela: Caucagua (MNHNP); Flint, McAlpine y Ross 1987: 3436 (sinonimia).

Diagnosis.-Adultos (Fig. $1 \mathrm{C}$ ): Moderamente grandes y robustos. Cabeza, tórax y patas cubiertos densamente de pelillos pardos, y con las alas translúcidas de color pardo. Ala anterior de 16 a $20 \mathrm{~mm}$ de longitud, con dos puntos pequeños y oscuros, uno sobre la base de la vena costa y el otro sub-basalmente en la vena subcosta, el margen anterior ligeramente cubierto apicalmente de pelillos oscuros, y una banda de pelillos pardo oscuros sobre el "cord".

Phallus (Fig. 5): Esta especie y L. divaricatum Flint, McAlpine y Ross, son muy similares, sin embargo pueden ser reconocidas por la forma de la sección apical del phallus, y por la forma y tamaño de los procesos dorsal y ventral del conducto eyaculatorio del phallus, como se detalla en la diagnosis de $L$. divaricatum. De acuerdo con los datos de distribución, estas dos especies solapan sus áreas de distribución, siendo $L$. crassum más frecuente en tierras bajas, como fue señalado por Flint $e t$ al. (1987). Asimismo, las colectas examinadas para esta revisión, indican que esta especie fue mucho más abundante que $L$. divaricatum. Procesos " $a$ ", " $b$ ", " c $c$ ", " $d$ ", " $e$ ", " $f$ ', " g" y " "j" ausentes en $L$. crassum.

Distribución: MéXico; Guatemala; Honduras; Nicaragua; Costa Rica (Alajuela, Guanacaste, Heredia, Limón, Puntarenas, San José); Panamá; Colombia; Venezuela; Brasil; Perú; Paraguay; Argentina.

Material examinado: CosTA RICA: Guanacaste: Río Tempisquito, ca. $3 \mathrm{~km} \mathrm{~S}$ route 1 , $10.790^{\circ} \mathrm{N}, 85.552^{\circ} \mathrm{W}$, el. $75 \mathrm{~m}, 6$. iii.1986, Holzenthal, Fasth, $30^{\prime \prime}$ (1 O’, $1 \%$ en alcohol); igual pero, $10.847^{\circ} \mathrm{N}, \quad 85.561^{\circ} \mathrm{W}$, el. $95 \mathrm{~m}$, 18.vii.1987, Holzenthal, Morse, de la Rosa, 35 ơ, 19 \&; 6.vi.1988, E. de la Rosa, 25 O' (en alcohol); P. N. Sta. Rosa, Río Poza Salada, $10.799^{\circ} \mathrm{N}, 85.652^{\circ} \mathrm{W}$, el. $10 \mathrm{~m}, 24 . v i i .1987$,
Holzenthal, Morse, Clausen, 10 o', 1 \%; Heredia: Río Sarapiquí, $7 \mathrm{~km}$ W Pto Viejo, $10.452^{\circ} \mathrm{N}$, $84.067^{\circ} \mathrm{W}$, el. $50 \mathrm{~m}$, 11.ii.1986, Holzenthal, Morse, Fasth, 6 o", 2 \%; Río Sarapiquí, $300 \mathrm{~m} \mathrm{~S}$ de Chilamate, $10.451^{\circ} \mathrm{N}, 84.064^{\circ} \mathrm{W}$, el. $40 \mathrm{~m}$, 30.vi.1992, Muñoz, 3 ơ, 2 ९; Est. Biol. La Selva, Río Puerto Viejo, $10.440^{\circ} \mathrm{N}, 84.012^{\circ} \mathrm{W}$, el. 30 m, 10-11.ii.1986, Holzenthal, Morse, Fasth, 28 ơ, 17 \&; igual pero, 19.vi.1986, Holzenthal, Heyn, Armitage, 6 o'; 10.ix.1986, M.M. Chavarría, 5 ơ, 3 \%; xii.1986, 6 ơ; i.1987, 6 ơ, 5 ᄋ; Limón: Río Telire and small trib., SE Suretka, $9.554^{\circ} \mathrm{N}, 82.892^{\circ} \mathrm{W}$, el. $48 \mathrm{~m}$, 1.ii. 1986 , Holzenthal, Morse, Fasth, 7 ơ, 13 ( 1 ơ, 6 \&n alcohol); Res. Biol. Hitoy Cerere, Río Cerere, $9.671^{\circ} \mathrm{N}, 83.028^{\circ} \mathrm{W}$, el. $90 \mathrm{~m}, 23-24 . i i i .1987$, Holzenthal, Hamilton, Heyn, 1 ơ, 3 \%; Río Barbilla, ca. $8 \mathrm{~km}$ W B-Line, $10.067^{\circ} \mathrm{N}, 83.369^{\circ} \mathrm{W}$, el. 30 m, 31.i.1986, Holzenthal, Morse, Fasth, 1 O' (en alcohol); E.A.R.T.H., Río Dos Novillos, $10.220^{\circ} \mathrm{N}, 83.591^{\circ} \mathrm{W}$, el. $20 \mathrm{~m}$, 3.ii.1992, Holzenthal, Muñoz, Kjer, 4 ○’, 4 \%; igual pero, Río Parismina, $10.248^{\circ} \mathrm{N}, 83.570^{\circ} \mathrm{W}$, el. $5 \mathrm{~m}$, 4.ii.1992, Holzenthal, Muñoz, Kjer, 2 ơ (en alcohol); Puntarenas: Queb. Pita, ca. 3 km (air) $\mathrm{W}$ Golfito, $8.642^{\circ} \mathrm{N}, 83.193^{\circ} \mathrm{W}$, el. $15 \mathrm{~m}$, 15.ii.1986, Holzenthal, Morse, Fasth, 4 ơ (1 en alcohol); Río Rincón, 6.5 km (air) S Rincón, $8.638^{\circ} \mathrm{N}, 83.480^{\circ} \mathrm{W}$, el. $20 \mathrm{~m}$, 7.iv.1987, Holzenthal, Hamilton, Heyn, 34 ơ, 5 \%; Río Catarata, $5.5 \mathrm{~km} \mathrm{~S}$ El Brujo (ca. Zona Sur), $9.064^{\circ} \mathrm{N}$, $83.272^{\circ} \mathrm{W}$, el. 120 m, 6-7.vii.1992, Muñoz, $10^{\circ}$, 1 \&; P. N. Corcovado, Río Claro, 1.5 km SE Sirena, $8.472^{\circ} \mathrm{N}, 83.588^{\circ} \mathrm{W}$, el. $15 \mathrm{~m}$, 8.iv. 1989 , Holzenthal, Blahnik, 9 ơ, 2 \%; Est. Sirena, Río Camaronal, $8.481^{\circ} \mathrm{N}, 83.594^{\circ} \mathrm{W}$, el. $5 \mathrm{~m}, 12-$ 13.iv.1989, Holzenthal, Blahnik, 3 ơ (en alcohol); igual pero, $8.482^{\circ} \mathrm{N}, 83.589^{\circ} \mathrm{W}$, el. $30 \mathrm{~m}$, 13.iv.1989, 2 O’; Península de Osa, 4-12.v.1979, D.H. Janzen, 3 O’, 1 \&; Res. Biol. Carara, Río Carara, $4.3 \mathrm{~km}$ (rd) E Costanera Sur, $9.810^{\circ} \mathrm{N}$, $84.572^{\circ} \mathrm{W}$, el. $20 \mathrm{~m}, 12 . i i i .1991$, Holzenthal, Muñoz, Huisman, $10^{7}$; Río Platanar, Salitre, 6.5 $\mathrm{km}$ NE Buenos Aires, $9.115^{\circ} \mathrm{N}, 83.281^{\circ} \mathrm{W}$, el. 450 m, 8-9.vi.1992, Muñoz, 1 ơ, 2 \&; San José: Res. Biol. Carara, Río Carara in Carara, 9.778 $\mathrm{N}, 84.531^{\circ} \mathrm{W}$, el. $200 \mathrm{~m}, 14$. iii. 1991 , Holzenthal, Muñoz, Huisman, 1 ơ, 3 ㅇ. 


\section{Leptonema divaricatum Flint, McAlpine y Ross}

Fig. 6; Mapa 4.

Leptonema divaricatum Flint, McAlpine y Ross 1987: 36, figs. 234-242, ơ, Ecuador: Pichincha (NMNH); Holzenthal 1988: 67.

Leptonema crassum Mosely 1933: 12-13 (identificación errónea de especímenes de Cachabé, Ecuador, según Flint et al., 1987); Fischer 1947: 313-315 (identificación errónea, según Flint et al., 1987); Schmid 1964: 317 (identificación errónea, según Flint et al., 1987); Flint 1981: 20, figs. 71-72 (identificación errónea, según Flint et al., 1987).

Diagnosis.-Adultos: Moderamente grandes y robustos. Cabeza, tórax y patas cubiertos densamente de pelillos pardos, y con las alas translúcidas de color pardo. Ala anterior de 16 a $20 \mathrm{~mm}$ de longitud, con dos puntos pequeños y oscuros, uno sobre la base de la vena costa y el otro sub-basalmente en la vena subcosta, el margen anterior ligeramente cubierto apicalmente de pelillos oscuros, y una banda de pelillos pardo oscuros sobre el "cord" (similar a $L$. crassum, Fig. 1 C).

Phallus (Fig. 6): Esta especie aunque muy similar a L. crassum Ulmer (Fig. 5), puede distinguirse por la forma de la sección apical del phallus, y por la forma y tamaño de los procesos dorsal y ventral del conducto eyaculatorio del phallus. Sección apical del phallus, en $L$. divaricatum, en vista posterodorsal, con una abertura dorsoapical, redondeada y bordeada por una membrana conspicua, erguida y dirigida posteriormente, exhibiendo una hendidura mesodorsal delante de los procesos dorsal y ventral del conducto eyaculatorio; conducto eyaculatorio en vista lateral, mesial, tubular y bifurcado apicalmente; proceso dorsal en vista posterodorsal, mesial, corto y en forma de una "U", con los ápices puntiagudos, en vista lateral, curvado levemente y alargado, sobrepasando levemente el margen dorsal del phallus; proceso ventral en vista posterodorsal, mesial, ancho, largo y truncado levemente, en vista lateral, fuertemente curvado basalmente y seguidamente erguido, digitiforme y largo, más largo que el proceso dorsal y sobrepasa el margen dorsoapical del phallus. Sección apical del phallus, en L. crassum, en vista posterodorsal, con una abertura dorsoapical, redondeada y bordeada por una membrana corta, empequeñecida mesodorsalmente delante de los procesos dorsal y ventral del conducto eyaculatorio; conducto eyaculatorio en vista lateral, mesial, tubular y bifurcado apicalmente; proceso dorsal en vista posterodorsal, mesial, corto y subtriangular, en vista lateral, corto y dirigido dorsalmente sin alcanzar el margen dorsal del phallus; proceso ventral en vista posterodorsal, mesial, corto y subtriangular, en vista lateral, sinuoso levemente, angular en la mitad y proyectado posterodorsalmente, apenas alcanza el margen dorsoapical del phallus (más largo que el proceso dorsal, pero más corto que el proceso ventral de $L$. divaricatum). Estas dos especies solapan sus ámbitos de distribución, como es comentado en la diagnosis de L. crassum. En L. divaricatum, procesos " $a$ ", " $b$ ", "c", " d", "e", "f', "g" y "j" ausentes.

Distribụción: COSTA RICA (Alajuela, Heredia, Limón); Colombia; VenEZUELA; EcUAdOR.

Material examinado: Costa RICA: Alajuela: Río Pizote, ca. $5 \mathrm{~km} \mathrm{~N}$ Dos Ríos, $10.948^{\circ} \mathrm{N}, 85.291^{\circ} \mathrm{W}$, el. $470 \mathrm{~m}, 9 . i i i .1986$, Holzenthal, Fasth, $1 \mathrm{O}^{7}$; Heredia: P. N. Braulio Carrillo, Est. Magsasay, Río Peje, $10.402^{\circ} \mathrm{N}$, $84.050^{\circ} \mathrm{W}$, el. $130 \mathrm{~m}, 25-26$.viii.1990, Holzenthal, Blahnik, Huisman, $10^{\prime}, 2$ 우 quí, $300 \mathrm{~m} \mathrm{~S}$ de Chilamate, $10.451^{\circ} \mathrm{N}$, $84.064^{\circ} \mathrm{W}$, el. $40 \mathrm{~m}, 30 . v i .1992$, Muñoz, $10^{\circ}$.

\section{Leptonema tapanti Muñoz-Q.}

Fig. 7; Mapa 2.

Leptonema tapanti Muñoz-Q. 1997: 127130, fig. 5, ơ', Costa Rica: Cartago (NMNH).

Diagnosis.-Adultos: Moderamente grandes y con las alas translúcidas de color pardo claro. Ala anterior de 17 a $20 \mathrm{~mm}$ de longitud, cubierta ligeramente de pelillos pardos principalmente entre las venas anales, y con un parche pequeño y redondeado de pelillos pardo oscuros sobre cada "nygma". 
Phallus (Fig. 7): Es L. tapanti la primera especie del Grupo stigmosum presente en Costa Rica. Esta especie difiere de L. auriculatum Flint, McAlpine y Ross del mismo Grupo, y de las siguientes especies del Grupo plicatum: $L$. michoacanense Flint, McAlpine y Ross, L. plicatum Mosely, y L. simplex Mosely, por la forma de los procesos " $b$ ", " $c$ " y " $e$ ", y por la presencia de los procesos " $d$ " y " $g$ ". En L. tapanti, procesos " $b$ " y " $c$ " están fusionados en un lóbulo apicolateral pareado (" $b-c$ "), en vista dorsal, la parte media del lóbulo " $b-c$ " es cóncava dorsolateralmente, el margen lateral curvado convexamente y ensanchado con el ápice puntiagudo levemente y dirigido posteriormente, semejando una oreja, con una proyección mediolateral, delgada, alargada, curvada y proyectada mesialmente. En las otras especies, procesos " $b$ " y " $c$ " no están fusionados y sin ninguna proyección mediolateral. Proceso " $d$ ", en $L$. tapanti, L. michoacanense, L. plicatum y L. simplex, ausente; y en L. auriculatum, es mesodorsal y rudimentario. Proceso " $e$ ", en esta especie, aparece dorsolateral y desarrollado en tres filas alargadas de espinas pequeñas y fusionadas a la sección media del phallus; la fila mesodorsal en forma de "Y", las otras, dos dorsolaterales y con los extremos anteriores desarrollados en lóbulos membranosos y redondeados; en L. auriculatum, es dorsolateral y muy pequeño; en $L$. michoacanense y $L$. plicatum, un lóbulo dorsolateral, alargado sin espinas pequeñas y fusionado a la sección media del phallus; y en L. simplex, ausente. Proceso " $g$ ", en L. tapanti, ausente; en L. michoacanense y $L$. plicatum, un lóbulo apicolateral y ensanchado; y en L. simplex, un lóbulo lateral, truncado y ensanchado. En L. tapanti, procesos " $d$ ", " $f$ ", " $g$ " y " $j$ " ausentes.

Distribución: COSTA RICA (Cartago); PANAMÁ.

Material examinado: Costa RicA: Cartago: Res. Tapantí, Queb. Palmitos and falls, $9.72^{\circ} \mathrm{N}, 83.78^{\circ} \mathrm{W}, 1400 \mathrm{~m}, 24-25$. iii.1991, Holzenthal, Muñoz, Huisman, ơ, holotipo (NMNH); igual pero, 10 ơ, 2 \%; 21.ii.1992, Holzenthal, Muñoz, Kjer, 1 ơ, 3 \& (INBio); Río Grande de Orosi, $9.686^{\circ} \mathrm{N}, 83.756^{\circ} \mathrm{W}$, el.
1650 m, 18-21.iii.1987, Holzenthal, Hamilton, Heyn, 1 ơ, 1 \%; Río Dos Amigos and falls, ca. $6 \mathrm{~km}$ (road) NW tunnel, $9.704^{\circ} \mathrm{N}, 83.783^{\circ} \mathrm{W}$, el. 1500 m, 23.iii.1991, Holzenthal, Muñoz, Huisman, 1 o", 1 \%.

\section{Leptonema ekisi Flint, McAlpine y Ross Fig. 8; Mapa 1.}

Leptonema ekisi Flint, McAlpine y Ross 1987: 54, figs. 463-470, ơ, Panamá: Chiriquí (NMNH); Holzenthal 1988: 67.

Leptonema salvini Mosely 1993: 64 (identificación errónea del paratipo ơ de Orosi, Cartago, Costa Rica, según Flint et al., 1987).

Diagnosis.-Adultos: Grandes y con las alas translúcidas de color pardo. Ala anterior de 22 a $29 \mathrm{~mm}$ de longitud, con tres parches pequeños y redondeados de pelillos pardo oscuros, uno sobre cada "nygma" y el tercero sobre la vena transversal " $m_{2}-m_{3}$ ".

Phallus (Fig. 8): Esta especie, L. fortunum Flint, McAlpine y Ross, $L$. rafita Muñoz-Q., $L$. salvini Mosely, y L. vitum Flint, McAlpine y Ross, pueden ser distinguidas por la forma y tamaño de los procesos " $e$ ", " $f$ " y " $g$ " como se comenta en la diagnosis de $L$. rafita. Procesos " $c$ ", " $d$ " y " $j$ " ausentes en esta especie.

Distribución: Costa Rica (Alajuela, Cartago); PANAmá.

Material examinado: CostA RICA: Cartago: Res. Tapantí, Río Grande de Orosi, $9.686^{\circ} \mathrm{N}, 83.756^{\circ} \mathrm{W}$, el. $1650 \mathrm{~m}$, 8-9.vii.1986, Holzenthal, Heyn, Armitage, $10^{\circ}, 11$ \%; igual pero, 18-21.iii.1987, Holzenthal, Hamilton, Heyn, 16 ơ, 13 \%; 15-16.vii.1987, Holzenthal, Morse, Clausen, $10^{\prime}, 1$ (en alcohol).

\section{Leptonema flintorum Muñoz-Q.} Fig. 17; Mapa 2.

Leptonema flintorum Muñoz-Q. 1997: 119-120, fig. 2, ơ, Costa Rica: Puntarenas (NMNH):

Diagnosis.-Adultos: Moderamente grandes y con las alas translúcidas de color pardo 

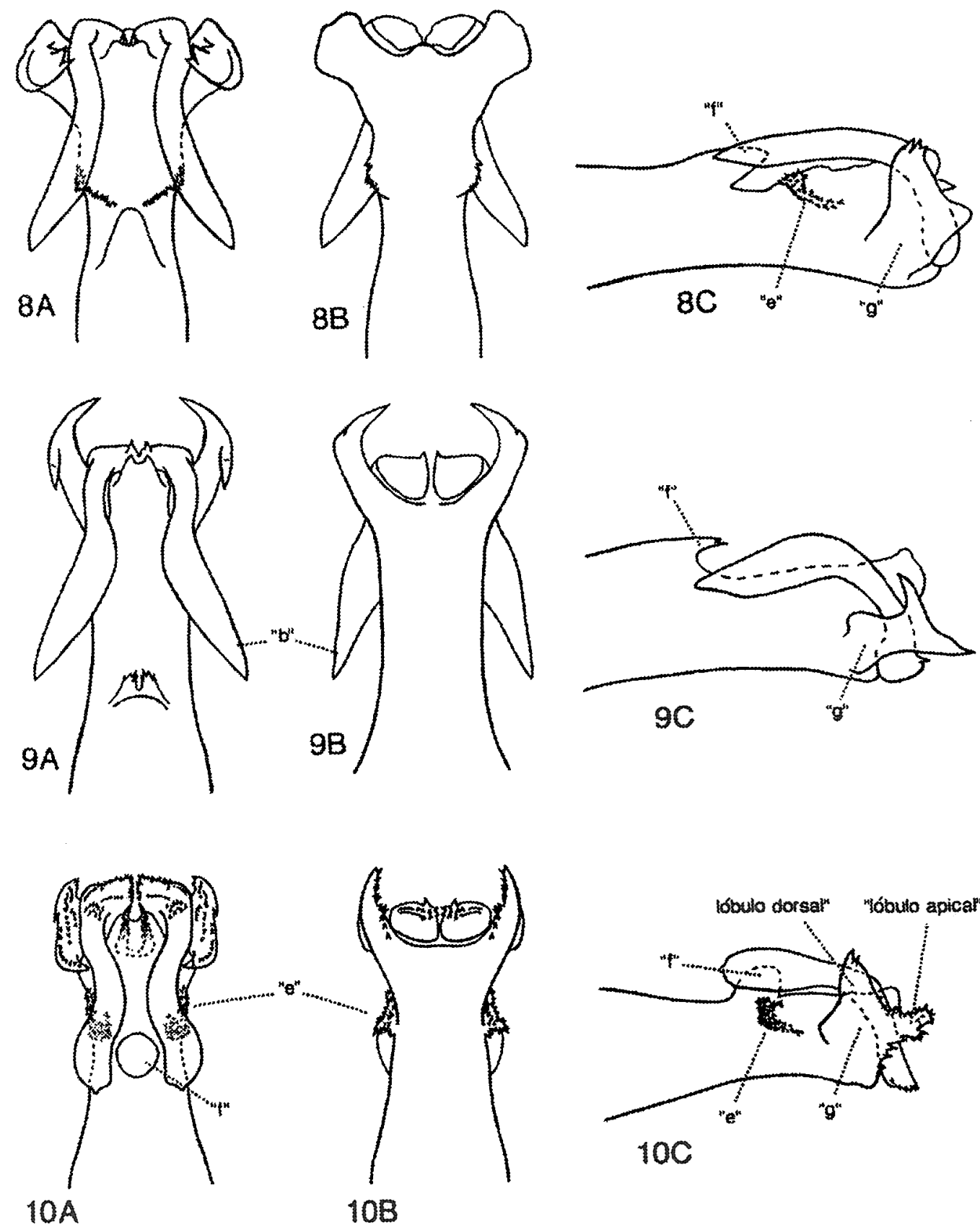

Figs. 8-10. Phallus: 8. L. ekisi, 9. L. fortunum, 10. L. rafita. -A., vista dorsal; B., vista ventral; C., vista lateral. 

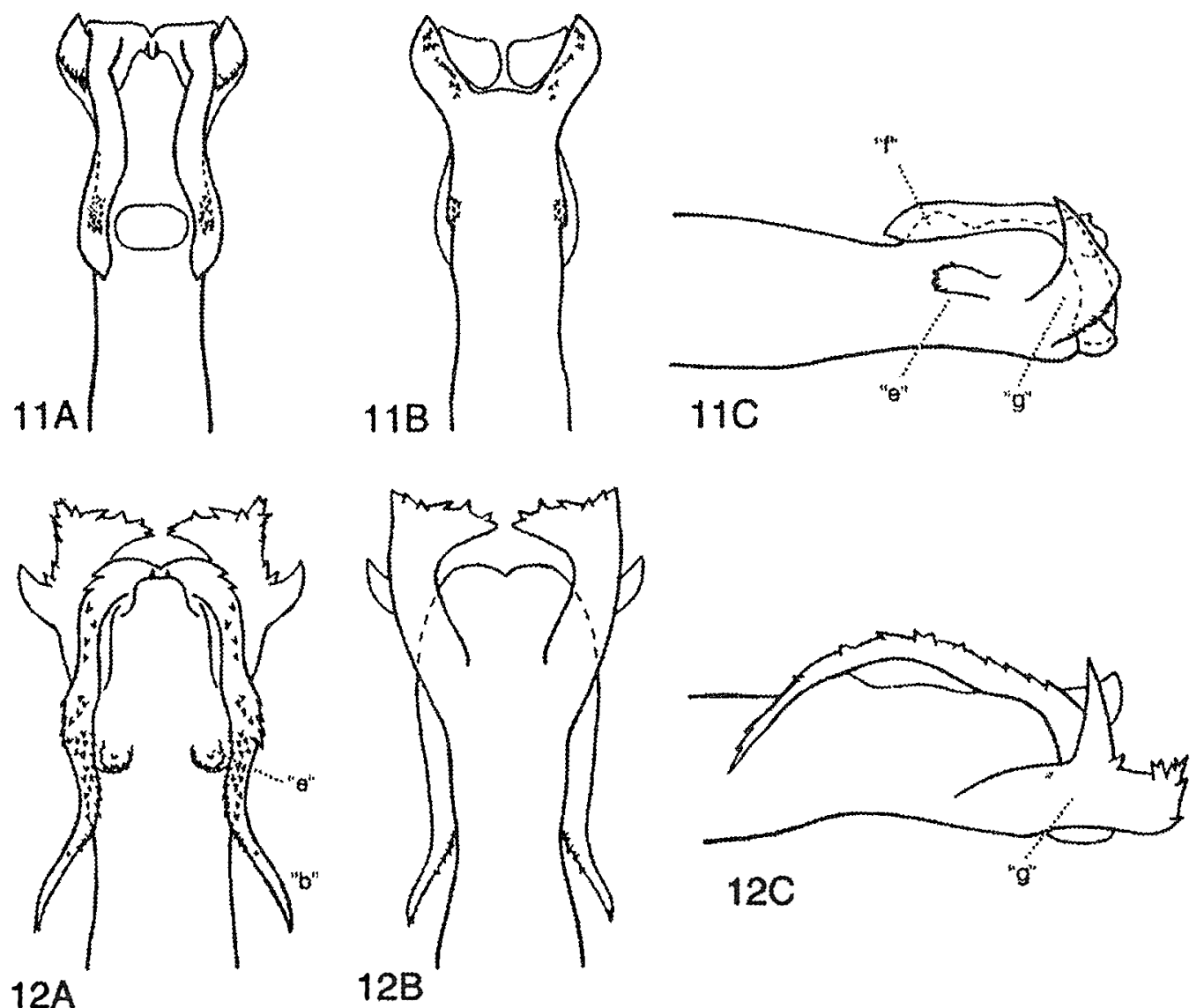

$12 \mathrm{~A}$

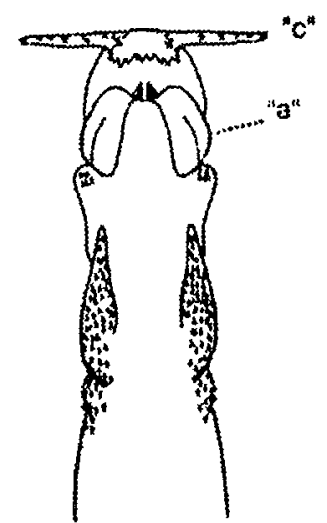

$13 \mathrm{~A}$
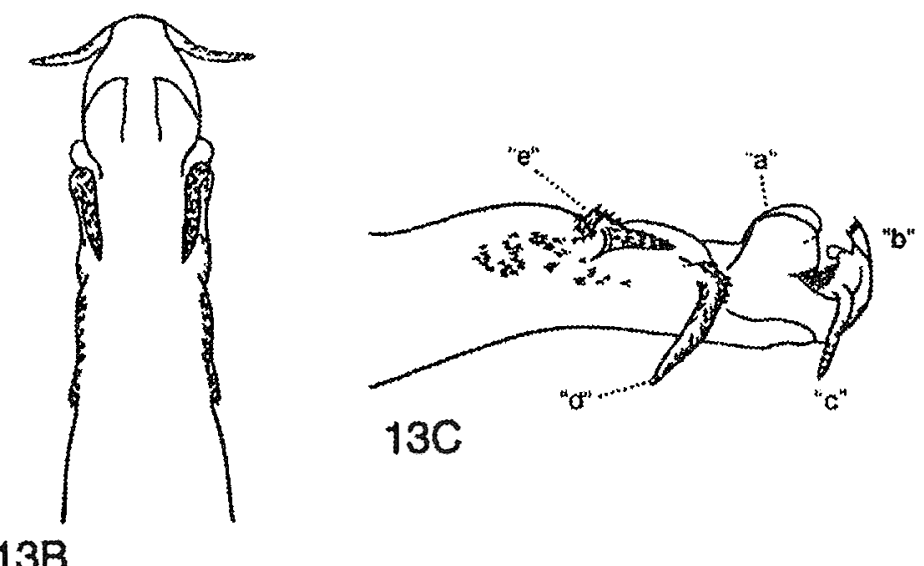

Figs. 11-13. Phallus: 11. L. salvini, 12. L. vitum, 13. L. albovirens. -A., vista dorsal; B., vista ventral; C., vista lateral. 


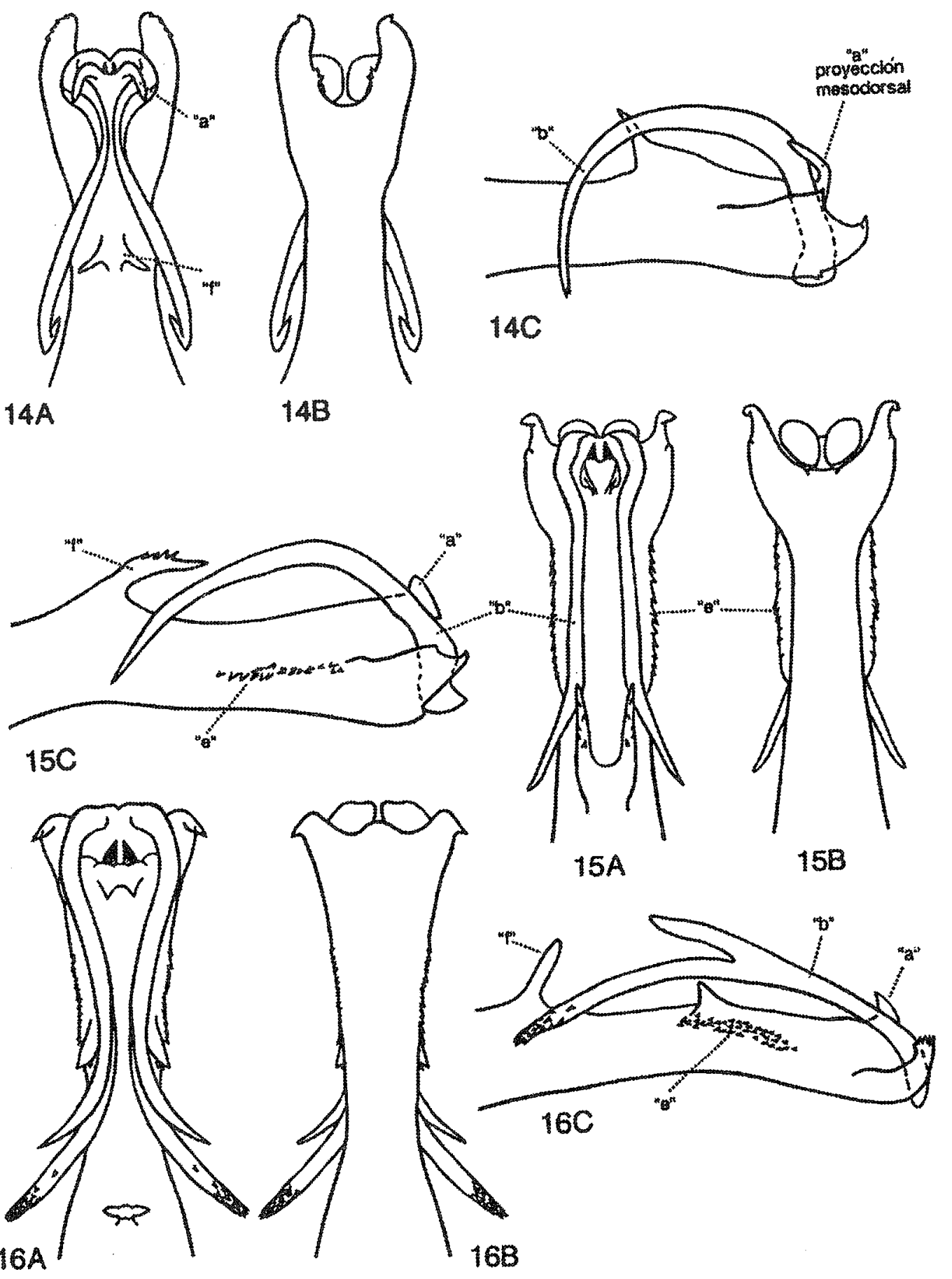

Figs. 14-16. Phallus: 14. L. sinuatum, 15. L. turrialbum, 16. L. woldianum. -A., vista doral; B., vista ventral; C., vista lateral. 
claro. Ala anterior de 20 a $22 \mathrm{~mm}$ de longitud, con pelillos pardo oscuros a lo largo de las venas " $A_{1}$ " y " $A_{2}$ ", y una banda de pelillos pardo oscuros sobre el "cord".

Phallus (Fig. 17): Esta especie aunque muy similar a L. huismanae Muñoz-Q. (Fig. 19), difiere en el tamaño de la sección media del phallus y del proceso " $e$ ", y en la forma y tamaño del proceso " $g$ ". Sección media del phallus, en $L$. flintorum, levemente más angosta que la sección apical del phallus, esta última sección es menos de 1.5 veces el ancho de la sección media; y en L. huismanae, notablemente. más estrecha que la sección apical del phallus, esta última sección es más de 2 veces el ancho de la sección media. Proceso " $e$ ", en $L$. flintorum, robusto y conspicuo, en vista lateral, la altura de la sección media del phallus es menos de 2.5 veces el largo del proceso " $e$ "; y en L. huismanae, de pequeño a diminuto, en vista lateral, la altura de la sección media del phallus es más de 3 veces el largo del proceso " $e$ ". Proceso " $g$ ", en esta especie, en vista dorsal, angosto, fuertemente cóncavo dorsalmente y alargado, alcanza el margen posterior del proceso " $b$ ", en vista lateral, el ápice aserrado levemente y redondeado levemente; y en L. huismanae, en vista dorsal, ensanchado, levemente cóncavo dorsalmente y alargado, casi siempre proyectado más allá del margen posterior del proceso " $b$ " (en algunos ejemplares, éste llega hasta el margen posterior del proceso " $b$ "), el ápice aserrado, amplio y truncado ligeramente. Estas dos especies pueden ser separadas de L. sinuatum Mosely (Fig. 14), por la forma del proceso " $a$ " y por la presencia de los procesos " $e$ " y " $f$ ", estos dos últimos procesos también la distinguen de $L$. hamuli Flint, McApline y Ross (Fig. 18). Proceso " $a$ ", en L. flintorum y L. huismanae, sin ninguna proyección mesodorsal, como sí aparece y se describe en la diagnosis de $L$. sinuatum. Proceso " $e$ ", en L. flintorum y L. huismanae, semeja una espina con el ápice dirigido anterodorsalmente; en $L$. sinuatum y $L$. hamuli, ausente. Proceso ' $f$ ', en L. flintorum y $L$. huismanae, ausente; en $L$. sinuatum y $L$. hamuli, en vista dorsal, bifurcado y con las ramas dirigidas lateralmente. En L. flintorum, procesos " $c$ ", " $d$ ", " $f$ " y " $j$ " ausentes.

Distribución: Costa Rica (Puntarenas).

Material examinado: CosTA RICA: Puntarenas: Río Bellavista, ca. $1.5 \mathrm{~km}$ NW Las Alturas, $8.951^{\circ} \mathrm{N}, 82.846^{\circ} \mathrm{W}$, el. 1400 m, 16-17.iii.1991, Holzenthal, Muñoz, Huisman, O’, holotipo (NMNH); igual pero, 1 O", 4 \%; trib. Río Bellavista, Las Alturas (road to quarry) $8.952^{\circ} \mathrm{N}, 82.848^{\circ} \mathrm{W}$, el. 1480 m, 19.iii. 1991, Holzenthal, Muñoz, Huisman, 10 o".

\section{Leptonema fortunum Flint, McAlpine y Ross} Fig. 9; Mapa 1.

Leptonema fortunum Flint, McAlpine y Ross 1987: 54-55, figs. 471-478, ơ, Panamá: Chiriquí (NMNH); McElravy et al. 1981: 153; 1982: 307, 310, 312 (especie "A" de Leptonema no descrita); Holzenthal 1988: 67.

Diagnosis.-Adultos: De tamaño mediano y con las alas translúcidas de color pardo. Ala anterior de 15 a $19 \mathrm{~mm}$ de longitud, con un parche pequeño y redondeado de pelillos pardo oscuros sobre las venas transversales " $m_{2}-m_{3}$ " y " $m_{3}-m_{4}$ ".

Phallus (Fig. 9): Esta especie, L. ekisi Flint, McAlpine y Ross, L. rafita Muñoz-Q., L. salvini Mosely, y L. vitum Flint, McAlpine y Ross, pueden ser separadas por la forma y tamaño de los procesos " $e$ ", " $f$ " y " $g$ ", como se especifica en la diagnosis de L. rafita. Procesos " $c$ ", " $d$ ", " $e$ " y " $j$ " ausentes en L. fortunum.

Distribución: CosTa Rica (Cartago); PANAMÁ.

Material examinado: CostA RicA: Cartago: Res. Tapantí, Queb. Segunda, @ administration building, $9.761^{\circ} \mathrm{N}, 83.787^{\circ} \mathrm{W}$, el. 1250 m, 9-10.v.1990, Holzenthal, Blahnik, 2 $\mathrm{O}^{7}$; igual pero, Queb. Palmitos \& falls, $9.72^{\circ} \mathrm{N}$, $83.78^{\circ} \mathrm{W}$, el. $1400 \mathrm{~m}, 24-25$. iii.1991, Holzenthal, Muñoz, Huisman, 4 O’. 


\section{Leptonema hamuli Flint, McAlpine y Ross}

Fig. 18; Mapa 2.

Leptonema hamuli Flint, McAlpine y Ross 1987: 55, figs. 479-486, ơ, Panamá: Isla Barro del Colorado (NMNH); Holzenthal 1988: 67.

Diagnosis.-Adultos: Pequeños y con las alas translúcidas de color pardo claro. Ala anterior de 14 a $17 \mathrm{~mm}$ de longitud, y con una banda tenue de pelillos pardos sobre el "cord".

Phallus (Fig. 18): Esta especie es parecida a L. sinuatum Mosely (Fig. 14), pero distinguible por la forma y tamaño de los procesos " $a$ ", " $b$ " y " $c$ ". Proceso " $a$ ", en $L$. hamuli, aparece membranoso y sin proyecciones mesodorsales; y en $L$. sinuatum, con un par de proyecciones mesodorsales. Proceso " $b$ ", en $L$. hamuli, mesoapical, delgado y sinuoso levemente, en vista lateral, arqueado, más largo que el proceso " $c$ " y apenas alcanza la base del proceso " $f$ "; y en $L$. sinuatum, apicoventral, delgado y sinuoso, en vista lateral, arqueado, largo y sobrepasa la base del proceso " $f$ ". Proceso " $c$ ", en L. hamu$l i$, apicolateral, delgado, curvado levemente y dirigido anteroventralmente; y en $L$. sinuatum, ausente. Esta especie se diferencia de $L$. flintorum Muñoz-Q. y L. huismanae MuñozQ., por la presencia de los procesos " $e$ " $\mathrm{y}$ "f", como se detalla en la diagnosis de $L$. flintorum. Procesos " $e$ " y " $j$ " ausentes en $L$. hamuli.

Distribución: Costa Rica (Cartago, Guanacaste, Limón); PANAMÁ.

Material examinado: Costa RICA: Guanacaste: Río Los Ahogados, $11.3 \mathrm{~km}$ ENE Quebrada Grande, $10.865^{\circ} \mathrm{N}, 85.423^{\circ} \mathrm{W}$, el. 470 m, 26.vi.1986, Holzenthal, Heyn, Armitage, 2 O’; P. N. Guanacaste, Est. Pitilla, Río Orosí, $10.991^{\circ} \mathrm{N}, 85.428^{\circ} \mathrm{W}$, èl. $700 \mathrm{~m}$, 22-25.v.1990, Holzenthal, Blahnik, 17 O’, 6 \$; Limón: P. N. Braulio Carrillo, Queb. González, $10.160^{\circ} \mathrm{N}, 83.939^{\circ} \mathrm{W}$, el. $480 \mathrm{~m}, 12-$ 14.v.1990, Holzenthal, Blahnik, 2 O' (1 en alcohol).

\section{Leptonema huismanae Muñoz-Q.}

Fig. 19; Mapa 2.

Leptonema huismanae Muñoz-Q. 1997: 122-124, fig. 3, O’, Costa Rica: Alajuela (NMNH).

Diagnosis.-Adultos: Moderamente grandes y con las alas translúcidas de color pardo claro. Ala anterior de 17 a $20 \mathrm{~mm}$ de longitud, con una banda pequeña y transversal de pelillos pardo oscuros sobre el tercio basal de la ala, otra banda más oscura de pelillos pardos oscuros sobre el "cord" y sobre los márgenes de la celda medial $(\mathrm{cm})$.

Phallus (Fig. 19): Esta especie y L. flintorum Muñoz-Q. son muy parecidas, sin embargo pueden ser reconocidas por el tamaño de la sección media del phallus y del proceso " $e$ ", y por la forma y tamaño del proceso " $g$ ", como se comenta en la diagnosis de $L$. flintorum. Estas dos especies difieren de L. sinuatum Mosely, por la forma del proceso " $a$ " y por la presencia de los procesos " $e$ " y " $f$ ', estos dos últimos procesos la distinguen de L. hamuli Flint, McAlpine y Ross, como se especifica en las diagnosis de $L$. flintorum y $L$. sinuatum. Procesos " $c$ ", " $d$ ", " $f$ " y "j" ausentes en L. huismanae.

Distribución: Costa RICA (Alajuela, Guanacaste).

Material examinado: Costa RICA: Alajuela: Res. Forestal San Ramón, Río San Lorencito and tribs., $10.216^{\circ} \mathrm{N}, 84.607^{\circ} \mathrm{W}$, el. 980 m, 6-10.iii.1991, Holzenthal, Muñoz, Huisman, o", holotipo (NMNH); igual pero, 7 o", 10 \&; 13-16.vi.1986, C.M. \& O.S. Flint, Holzenthal, 11 ơ, 2 \& (NMNH); 2-4.vii.1986, Holzenthal, Heyn, Armitage, 3 ơ, 3 \&; 5-9.vii.1986, I. \& A. Chacón, 6 ơ, 2 \%; 2-6.ix.1986, 1 ơ, 1 \%; 24-27.ii.1987, 3 o', 1 \&; 30.iii.-1.iv.1987, Holzenthal, Halmilton, Heyn, 11 ơ (4 en alcohol), 5 \&; 1-4.v.1990, Holzenthal, Blahnik, 5 ơ (1 en alcohol), 12 \&; 28-30.vii.1990, Holzenthal, Blahnik, Muñoz, 1 ơ, 1 (en alcohol); Río Sarapiquí, ca. $2 \mathrm{~km}$ SE Cariblanco, $10.299^{\circ} \mathrm{N}$, 84.172 ${ }^{\circ} \mathrm{W}$, el. $710 \mathrm{~m}, 22$. .ii..1986, Holzenthal, Fasth, 1 ơ; igual pero, 6.ii.1987, I. \& A. Chacón, 2 ơ, 2 ९; Río Agrio, ca. 3.5 km NE Bajos 
REVISTA DE BIOLOGÍA TROPICAL

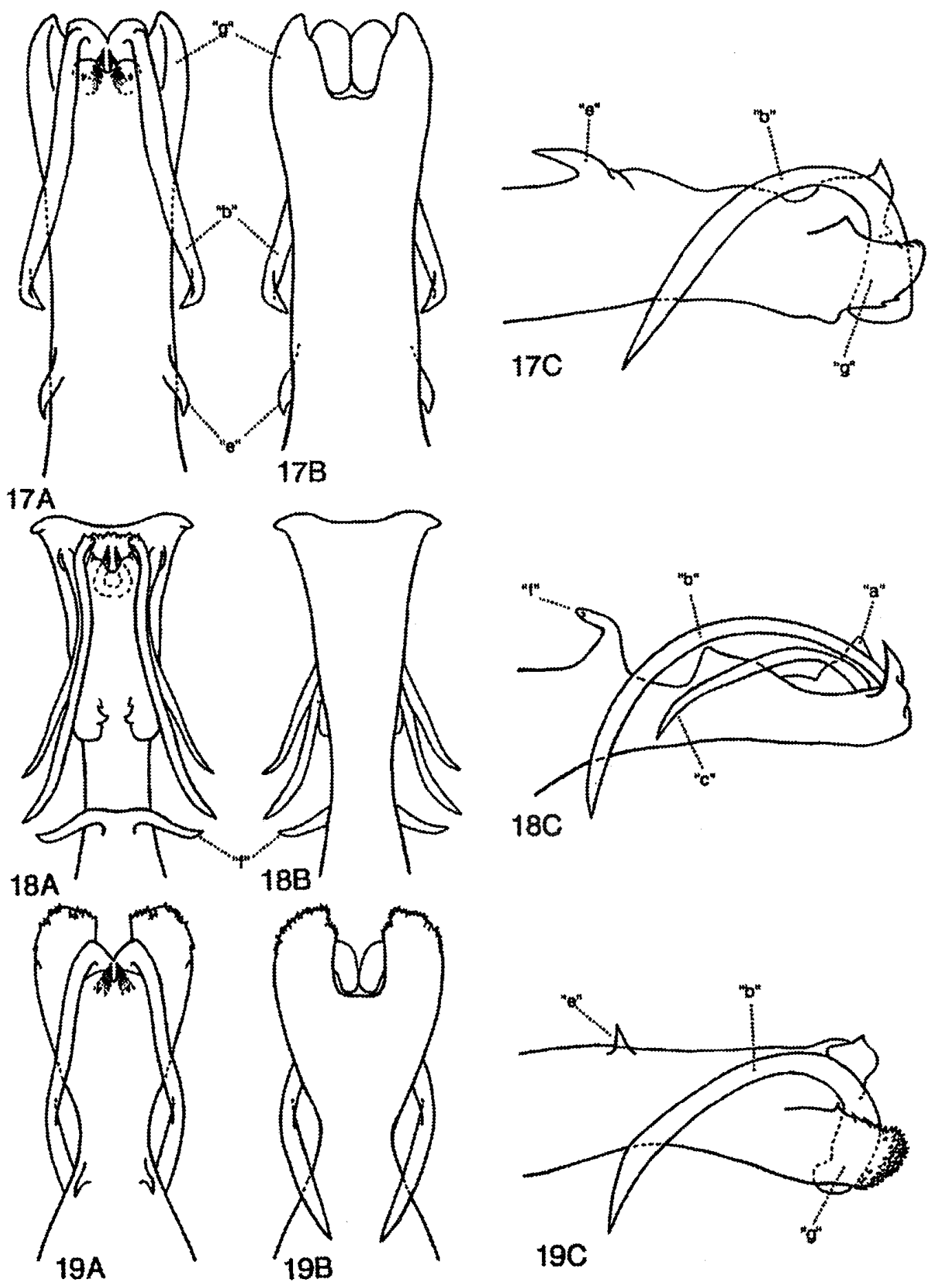

Figs. 17-19. Phallus: 17. L. flintorum, 18. L. hamuli, 19. L. huismanae. -A., vista dorsal; B., vista ventral; C., vista lateral. 
del Toro, $10.243^{\circ} \mathrm{N}, 84.279^{\circ} \mathrm{W}$, el. $1290 \mathrm{~m}$, 20.viii.1990, Holzenthal et al., 1 ơ; Guanacaste: P. N. Guanacaste, Río San Josecito, Est. Mengo [Est. Cacao], $10.922^{\circ} \mathrm{N}, 85.470^{\circ} \mathrm{W}$, el. 960 m, 28-29.vii.1987, Holzenthal, Morse, Clausen, 8 ơ, 4 \%; Est. Cacao, lado suroeste del Volcán Cacao, $\left[10.56^{\circ} \mathrm{N}, 85.26^{\circ} \mathrm{W}\right]$, el. 1000-1400 m, ix-xii.1989, URCG, R. Blanco, C. Chávez, 3 ơ (INBio); igual pero, vi.1990, II Curso de Parataxónomos, 14 O’, 12 \& (INBio); Zona Protectora Tenorio, tribs. Río San Lorenzo, $6 \mathrm{~km} \mathrm{NW}$ Tierras Morenas, $10.61^{\circ} \mathrm{N}$, $84.98^{\circ} \mathrm{W}$, el. $900 \mathrm{~m}, 17-19$. ii.1992, Holzenthal, Muñoz, Kjer, 3 ơ, 10 ㅇ.

\section{Leptonema rafita Muñoz-Q.}

Fig. 10; Mapa 1.

Leptonema rafita Muñoz-Q. 1997: 124127, fig. 4, ơ, Costa Rica: Alajuela (NMNH).

Diagnosis.-Adultos: Grandes y con las alas translúcidas de color pardo. Ala anterior de 20 a $23 \mathrm{~mm}$ de longitud, con un parche pequeño y redondeado de pelillos pardo oscuros sobre el margen posterior de celda medial $(\mathrm{cm})$.

Phallus (Fig. 10): Esta especie, L. ekisi Flint, McAlpine y Ross (Fig. 8), L. fortunum Flint, McAlpine y Ross (Fig. 9), L. salvini Mosely (Fig. 11) y L. vitum Flint, McAlpine y Ross (Fig. 12), pueden ser diferenciadas por la forma y tamaño de los procesos " $e$ ", " $f$ 'y " $g$ ". Proceso " $e$ ", en $L$. rafita y $L$. salvini, en vista dorsal, un lóbulo dorsolateral con minúsculas puntas superficiales y fusionado a la base del phallus, en vista lateral, proyectado anteriormente alcanzando la base del proceso " $f$ "; en $L$. ekisi, en vista dorsal, un lóbulo dorsolateral con minúsculas puntas superficiales y fusionado a la base del phallus, en vista lateral, corto y dirigido anteriormente sin alcanzar la base del proceso ' $f$ '; en $L$. fortunum, ausente; y en L. vitum, en vista dorsal, un lóbulo dorsal pequeño y redondeado, en vista lateral, corto y dirigido dorsalmente. Proceso " $f$ ", en esta especie, en vista dorsal, redondeado, en vista lateral, corto y erguido, con el ápice redondeado moderadamente y dirigido dorsalmente; en $L$. ekisi, en vista dorsal, alargado y con el ápice dirigido posteriormente, semejando una lengua; en $L$. fortunum, en vista dorsal, pequeño y dirigido posteriormente, con el ápice bifurcado levemente y las puntas aserradas tenuamente; en $L$. salvini, en vista dorsal, ovalado, en vista lateral, corto, con el ápice redondeado ligeramente y dirigido dorsalmente; y en L. vitum, ausente. Proceso " $g$ ", en $L$. rafita, corto y bilobulado; el lóbulo apical, en vista lateral, erguido, subtriangular, aserrado fuertemente y con un par de puntas apicales pequeñas, que alcanzan el margen posterior del proceso " $b$ "; el lóbulo dorsal erguido, subtriangular, aserrado y dirigido dorsalmente con el ápice puntiagudo. En L. ekisi, proceso " $g$ " corto y bilobulado apicalmente; el lóbulo apical corto, redondeado levemente, no aserrado y apenas alcanza el margen posterior del proceso " $b$ "; el lóbulo dorsal erguido, subtriangular y dirigido dorsalmente con unas puntas pequeñas y apicales. En $L$. fortunum, proceso " $g$ " bilobulado apicalmente, alargado y sobrepasa el margen posterior del proceso " $b$ "; el lóbulo apical, en vista lateral, subtriangular, erguido, no aserrado y con el ápice puntiagudo; lóbulo dorsal erguido, delgado, no aserrado, con el ápice puntiagudo y dirigido dorsalmente. En L. salvini, proceso " $g$ " unilobulado, en vista lateral, corto y alcan$z a$ el margen posterior del proceso " $b$ ", el margen posteroventral aserrado ligeramente; sólo el lóbulo dorsal, en vista lateral, dorsoapical, subtriangular, erguido, no aserrado, con el ápice puntiagudo y dirigido dorsalmente. En $L$. vitum, proceso " $g$ " bilobulado, alargado y sobrepasa el margen posterior del proceso " $b$ "; el lóbulo apical, en vista lateral, conspicuo, subcuadrado y aserrado posterodorsalmente; el lóbulo dorsal lateromedial, en vista lateral, erguido, delgado, subtriangular y dirigido dorsalmente. Margen apical del proceso " $g$ " difiere en estas especies cuando es visto ventralmente, en $L$. rafita, con una hendidura profunda, amplia y aserrada lateralmente semejando una "U"; en L. ekisi, con hendidura superficial, ensanchada y no aserrada con una proyección pequeña y mesoapical; en L. fortunum, con una hendidu- 
ra profunda y no aserrada, seme jando una "U" levemente cerrada apicalmente; en L. salvini, con una hendidura amplia y no aserrada, semejando una "U"; y en L. vitum, con una hendidura profunda, convexa lateralmente, sub-acorazonada y no aserrada. Procesos “c", " $d$ ", " $j$ " ausentes en L. rafita.

Distribución: CosTA RICA (Alajuela, Cartago, San José).

Material examinado: CosTA RICA: Alajuela: Río Peje and falls, ca. $1 \mathrm{~km}$ SE San Vicente, Ciudad Quesada, $10.277^{\circ} \mathrm{N}, 84.388^{\circ} \mathrm{W}$, el. 1450 m, 14-15.ii.1992, Holzenthal, Muñoz, Kjer, ơ', holotipo (NMNH); igual pero, 4 o', 4 \&; Río Toro, $3.0 \mathrm{~km}$ (road) SW Bajos del Toro, $10.204^{\circ} \mathrm{N}, 84.316^{\circ} \mathrm{W}$, el. $1530 \mathrm{~m}, 11 . i \mathrm{i} .1992$, Holzenthal, Muñoz, Kjer, $10^{7}$; Cartago: Res. Tapantí, Río Grande de Orosi, $9.686^{\circ} \mathrm{N}$, $83.756^{\circ} \mathrm{W}$, el. $1650 \mathrm{~m}, 15-16 . v i i .1987$, Holzenthal, Morse, Clausen, $10^{\prime}$; igual pero, Queb. Palmitos and falls, $9.72^{\circ} \mathrm{N}, 83.78^{\circ} \mathrm{W}$, el. 1400 m, 1-2.viii.1990, Holzenthal, Blahnik, Muñoz, 1 ơ, 1 \&; 21.ii.1992, Holzenthal, Muñoz, Kjer, 1 ơ, 1 \& (INBio); waterfalls, ca. 1 $\mathrm{km}$ (road) $\mathrm{NW}$ tunnel, $9.69^{\circ} \mathrm{N}, 83.76^{\circ} \mathrm{W}$, el. 1600 m, 2-3.viii.1990, Holzenthal, Blahnik, Muñoz, 1 ơ (NMNH); San José: trib. to Queb. Caraigres $3.6 \mathrm{~km}$ (road) SW La Legua, $9.728^{\circ} \mathrm{N}, 84.125^{\circ} \mathrm{W}$, el. $1650 \mathrm{~m}, 23 . \mathrm{i} .1992$, Holzenthal, Kjer, Quesada, $20^{\prime}$.

\section{Leptonema salvini Mosely}

Fig. 11; Mapa 1.

Leptonema salvini Mosely 1933: 62-64, figs. 196-200, ơ, Panamá: Chiriquí (BMNH); Fischer 1963: 172; Flint, McAlpine y Ross 1987: 57, figs. 525-531; Holzenthal 1988: 67.

Diagnosis.-Adultos: Ligeramente grandes y con las alas translúcidas de color pardo claro. Ala anterior de 15 a $21 \mathrm{~mm}$ de longitud, con un parche pequeño y redondeado de pelillos pardos oscuros sobre el ángulo anterior de la celda medial $(\mathrm{cm})$, y una banda de pelillos pardo oscuros sobre el "cord".

Phallus (Fig. 11): Esta especie es muy abundante en el país y sa distingue de L. ekisi
Flint, McAlpine y Ross, L. fortunum Flint, McAlpine y Ross, L. rafita Muñoz-Q., y L. vitum Flint, McAlpine y Ross, por la forma y tamaño de los procesos "e", " $f$ ' y " $g$ ", como se menciona en la diagnosis de $L$. rafita. En $L$. salvini, procesos " $c$ ", " $d$ " y " $j$ ", ausentes.

Distribución: Costa RICA (Alajuela, Cartago, Puntarenas, San José); PANAmÁ.

Material examinado: COSTA RICA: Alajuela: Río La Paz Pequeña, route $9,7.8 \mathrm{~km} \mathrm{~N}$ Vara Blanca, $10.211^{\circ} \mathrm{N}, 84.166^{\circ} \mathrm{W}$, el. $1230 \mathrm{~m}$, 13.ii.1986, Holzenthal, Morse, Fasth, 10 o’, 30 १; Río Sarapiquí, ca. 2 km SE Cariblanco, $10.299^{\circ} \mathrm{N}, 84.172^{\circ} \mathrm{W}$, el. $710 \mathrm{~m}, 22$. iii.1986, Holzenthal, Fasth, 15 ơ (en alcohol); Res. Forestal San Ramón, Río San Lorencito \& tribs., $10.216^{\circ} \mathrm{N}, 84.607^{\circ} \mathrm{W}$, el. $980 \mathrm{~m}, 1-4 . v .1990$, Holzenthal, Blahnik, 1 ơ, 2 \&; igual pero, 610.iii.1991, Holzenthal, Muñoz, Huisman, 1 ơ, 4 ᄋ; Río Agrio, ca. 3.5 km NE Bajos del Toro, $10.243^{\circ} \mathrm{N}, \quad 64.279^{\circ} \mathrm{W}$, el. $1290 \mathrm{~m}$, 20.viii.1990, Holzenthal et al, 8 o’, 2 \%; Queb. Latas, $8.9 \mathrm{~km} \mathrm{NE} \mathrm{Bajos} \mathrm{del} \mathrm{Toro,} 10.269^{\circ} \mathrm{N}$, $84.260^{\circ} \mathrm{W}$, el. $1030 \mathrm{~m}$, 6.ix.1990, Holzenthal, Blahnik, Huisman, 23 ơ, 13 ㅇ; Río Toro, 3.0 $\mathrm{km}$ (road) SW Bajos del Toro, $10.204^{\circ} \mathrm{N}$, $84.316^{\circ} \mathrm{W}$, el. $1530 \mathrm{~m}, 3-4 . i x .1990$, Holzenthal, Blahnik, Huisman, 10 ơ, 13 o; igual pero, 11.ii.1992, Holzenthal, Muñoz, Kjer, 2 ơ, 4 \&; Río Peje \& falls, ca. $1 \mathrm{~km}$ SE San Vicente, $10.277^{\circ} \mathrm{N}, 84.388^{\circ} \mathrm{W}$, el. $1450 \mathrm{~m}, 14-$ 15.ii.1992, Holzenthal, Muñoz, Kjer, 2 ơ; Río Peje \& falls, ca. $1 \mathrm{~km}$ SE San Vicente, $10.277^{\circ} \mathrm{N}, 84.388^{\circ} \mathrm{W}$, el. $1450 \mathrm{~m}, \quad 14-$ 15.ii.1992, Holzenthal, Muñoz, Kjer, 1 o’, 3 ○; Río Peje \& falls, ca. $1 \mathrm{~km}$ SE San Vicente, $10.277^{\circ} \mathrm{N}, 84.388^{\circ} \mathrm{W}$, el. $1450 \mathrm{~m}, 14-$ 15.ii.1992, Holzenthal, Muñoz, Kjer, 1 ơ, 3 ○; Cartago: Res. Tapantí, Queb. Segunda, @ administration building, $9.761^{\circ} \mathrm{N}, 83.787^{\circ} \mathrm{W}$, el. 1250 m, 9-10.v.1990, Holzenthal, Blahnik, 13 ơ; igual pero, Queb. Palmitos \& falls, $9.72^{\circ} \mathrm{N}, 83.78^{\circ} \mathrm{W}$, el. $1400 \mathrm{~m}, 2-3 . v i .1990$, Holzenthal, Blahnik, Muñoz, 11 ơ, 15 \&; 2425.iii.1991, Holzenthal, Muñoz, Huisman, 4 O’; Puntarenas: Res. Bosque Nuboso Monteverde, Queb. Cuecha, $10.31^{\circ} \mathrm{N}, 84.79^{\circ} \mathrm{W}$, el. 1550 m, 28.ii.1986, Holzenthal, Fasth, 1 o'; 
Río Bellavista, ca. $1.5 \mathrm{~km} \mathrm{NW} \mathrm{Las} \mathrm{Alturas,}$ $8.951^{\circ} \mathrm{N}, 82.846^{\circ} \mathrm{W}$, el. $1400 \mathrm{~m}, 15-17 . v i .1986$, Holzenthal, Heyn, Armitage, 13 o", 9 \& ( $10^{\circ}, 1$ $\$$ en alcohol); igual pero, 8-9.iv.1987, Holzenthal, Hamilton, Heyn, 41 ơ, 57 \& (14 O', 12 O en alcohol); 2-3.viii.1987, Holzenthal, Morse, Clausen, 8 O’, 8 \& (4 O’, 3 \& en alcohol); 10 11.viii.1990, Holzenthal, Blahnik, Muñoz, 21 o', 49 ( $20^{\prime \prime}, 12$ \& e n alcohol); 16-17.iii.1991, Holzenthal, Muñoz, Huisman, 71 O’, 28 \&; trib. to Río Bellavista in Las Alturas (road to quarry), $8.952^{\circ} \mathrm{N}, \quad 82.848^{\circ} \mathrm{W}, \quad$ el. $1480 \mathrm{~m}, \quad 13-$ 14.viii.1990, Holzenthal, Blahnik, Muñoz, 43 ơ, 29 \& (3 ơ, 1 \& en alcohol); igual pero, 19.iii.1991, Holzenthal, Muñoz, Huisman, 15 o", 11 \&; Z. P. Las Tablas, Río Cotón, Sitio Cotón, $8.941^{\circ} \mathrm{N}, 82.787^{\circ} \mathrm{W}$, el. $1460 \mathrm{~m}$, 15.iv. 1989 , Holzenthal, Blahnik, 50 o’, 16 O ; Río Cotón, in Las Alturas, $8.938^{\circ} \mathrm{N}, 82.826^{\circ} \mathrm{W}$, el. $1360 \mathrm{~m}$, 16.ii.1986, Holzenthal, Morse, Fasth, 1 ơ, 2 \&; igual pero, 12.viii.1990, Holzenthal, Blahnik, Muñoz, 31 đ’, 8 \& (30 đ’, 4 \& en alcohol); 18.iii.1991, Holzenthal, Muñoz, Huisman, 174 o', 12 ९ (51 ơ, 4 ९ en alcohol); 24.ix.1991, Muñoz, 120,7 ( en alcohol); small spring seep to Río Cotón in Las Alturas, $8.938^{\circ} \mathrm{N}, 82.824^{\circ} \mathrm{W}$, el. 1380 m, 14.viii.1990, Holzenthal, Blahnik, Muñoz, 35 O' (en alcohol); San José: P. N. Braulio Carrillo, park headquares, $10.059^{\circ} \mathrm{N}$, $84.017^{\circ} \mathrm{W}$, el. $1650 \mathrm{~m}$, 7.vii.1986, Holzenthal, Heyn, Armitage, 3 o'; Río Parrita Chiquito, rt. $12,6.5 \mathrm{~km} \mathrm{SW}$ jct. rt. $2,9.703^{\circ} \mathrm{N}, 83.970^{\circ} \mathrm{W}$, el. 1990 m, 18.vi.1986, Holzenthal, Heyn, Armitage, 5 o"; Queb. Caraigres, 2.5 km (rd) SW La Legua, $9.734^{\circ} \mathrm{N}, 84.120^{\circ} \mathrm{W}$, el. $1470 \mathrm{~m}$, 22.i.1992, Holzenthal, Kjer, Quesada, 1 ơ; trib to Queb. Caraigres, $3.6 \mathrm{~km}$ (rd) SW La Legua, $9.728^{\circ} \mathrm{N}, 84.125^{\circ} \mathrm{W}$, el. $1650 \mathrm{~m}$, 23.i.1992, Holzenthal, Kjer, Quesada, $20^{\circ}, 3$ \%.

\section{Leptonema sinuatum Mosely}

Fig. 14; Mapa 2.

Leptonema sinuatum Mosely 1933: 59-61, figs. 184-189, O', Colombia: Isla Gorgona (BMNH); Fischer 1963: 172; Flint, McAlpine y Ross 1987: $57-$ 58, figs. 539-545; Holzenthal 1988: 68.
Diagnosis.-Adultos: Pequeños y con las alas translúcidas de color pardo claro. Ala anterior de 13 a $15 \mathrm{~mm}$ de longitud, y con una banda tenue de pelillos pardo oscuros sobre el "cord".

Phallus (Fig. 14): Esta especie se reconoce de L. flintorum Muñoz-Q. (Fig. 17), L. hamuli Flint, McAlpine y Ross (Fig. 18), L. huismanae Muñoz-Q. (Fig. 19), L. turrialbum Flint, McAlpine y Ross (Fig. 15) y L. woldianum Flint, McAlpine y Ross (Fig. 16), por la forma del proceso " $a$ ". Proceso " $a$ ", en $L$. sinuatum, en vista dorsal, con un par de proyecciones mesodorsales, conspicuas y delgadas, en vista lateral, curvadas basalmente y dirigidas anteriormente; y en las otras especies, sin proyecciones mesodorsales. También, L. sinuatum se distingue por la forma y tamaño de los procesos " $b$ ", " $c$ ", " $e$ " y " $f$ ', como se detalla en las diagnosis de L. hamuli, L. flintorum y L. turrialbum. En L. sinuatum, procesos "c", " $d$ ", " $e$ " y " $j$ " ausentes.

Distribución: COSTA RICA (Puntarenas, San José); Panamá; Colombia.

Material examinado: CoSTA RICA: Puntarenas: Río Guineal, ca $1 \mathrm{~km}$ (air) E Finca Helechales, $9.076^{\circ} \mathrm{N}, 83.092^{\circ} \mathrm{W}$, el. $840 \mathrm{~m}$, 4.viii. 1987 , Holzenthal, Morse, Clausen, 1 ơ, 3 ㅇ.

\section{Leptonema turrialbum Flint, McAlpine y Ross}

Fig. 15; Mapa 2.

Leptonema turrialbum Flint, McAlpine y Ross 1987: 58, figs. 546-553, ơ, Costa Rica: Cartago (NMNH); Holzenthal 1988: 68.

Diagnosis.-Adultos: Pequeños y con las alas translúcidas de color pardo. Ala anterior de 13 a $17 \mathrm{~mm}$ de longitud, con un punto pequeño y oscuro sobre la base de la vena costa, una banda pequeña y transversal de pelillos pardo oscuros sobre la unión de las venas " $A_{l}$ " y " $A_{2}$ ", y otra banda de pelillos pardo oscuros sobre el "cord".

Phallus (Fig. 15): Esta especie endémica aunque similar a L. sinuatum Mosely (Fig. 14) y L. woldianum Flint, McAlpine y Ross (Fig. 
16), difiere en la forma y tamaño de los procesos " $a$ ", " $b$ ", " $e$ " у " $f$ ". Proceso " $a$ ", en $L$. turrialbum y $L$. woldianum, sin proyecciones mesodorsales, sí presentes en $L$. sinuatum (como se describe en la diagnosis de esa especie). Proceso " $b$ ", en $L$. turrialbum, delgado y largo, en vista dorsal, con una curva pequeña y cerrada basalmente, en vista lateral, arqueado, no bifurcado y alcanzando la base del proceso " $f$ "; en $L$. sinuatum, delgado y largo, en vista dorsal, sinuoso, en vista lateral, arqueado, no bifurcado y sobrepasa la base del proceso " $f$ "; y en $L$. woldianum, delgado, largo, en vista dorsal, sinuoso levemente, en vista lateral, arqueado y bifurcado profundamente, con la rama dorsal más corta que la ventral, la rama ventral alcanza la base del proceso " $f$ '. Proceso " $e$ ", en L. turrialbum, fusionado a la base del phallus, en vista lateral, a manera de una fila larga de espinas pequeñas; en $L$. sinuatum, ausente; y en $L$. woldianum, fusionado a la base del phallus, en vista lateral, desarrollado en una fila larga de espinas pequeñas, el extremo anterior con un lóbulo subtriangular y dirigido dorsalmente. Proceso ' $f$ ', en L. turrialbum, en vista dorsal, alargado, bifurcado y con las ramas dirigidas posteriormente, en vista lateral, robusto, curvado y aserrado; en $L$. sinuatum, en vista dorsal, bifurcado y con las ramas dirigidas lateralmente; y en L. woldianum, en vista dorsal, bifurcado levemente y con las ramas pequeñas y dirigidas lateralmente. En L. turrialbum, procesos " $c$ ", " $d$ " y " $j$ " ausentes.

Distribución: COSTA Rica (Alajuela, Cartago, Heredia, Limón).

Material examinado: Costa RICA: Cartago: Río Chitaría, rt 10, 10 km NW Río Reventazón, $9.920^{\circ} \mathrm{N}, 83.604^{\circ} \mathrm{W}$, el. $740 \mathrm{~m}$, 21.iii.1991, Holzenthal, Muñoz, Huisman, 2 O', 7 \%; Heredia: P. N. Braulio Carrillo, Est. El Ceibo, Río Peje, $10.327^{\circ} \mathrm{N}, 84.078^{\circ} \mathrm{W}$, el. 480 m, 29-31.v.1990, Holzenthal, Blahnik, Muñoz, 2 ơ, 2 o; Puerto Viejo, Río Chilamate, $10.490^{\circ} \mathrm{N}, 84.100^{\circ} \mathrm{W}$, el. $75 \mathrm{~m}$, vi. 1990 , Fdo Muñoz, $10^{\prime \prime}, 1$ ㅇ (en alcohol); Limón: Res. Biol. Hitoy Cerere, Río Cerere, $9.671^{\circ} \mathrm{N}$, $83.028^{\circ} \mathrm{W}$, el. $90 \mathrm{~m}, 23-24$. iii. 1987 , Holzenthal, Hamilton, Heyn, 1 O’, 1 \%; P. N. Braulio
Carrillo, Queb. González, $10.160^{\circ} \mathrm{N}$, $83.939^{\circ} \mathrm{W}$, el. $480 \mathrm{~m}, 12-14 . v .1990$, Holzenthal, Blahnik, 3 ơ, 11 \& (1 O', 5 \& en alcohol); Res. Biol. Barbilla, Río Dantas, 15 km (rd) S Pacuarito, $9.994^{\circ} \mathrm{N}, 83.443^{\circ} \mathrm{W}$, el. $300 \mathrm{~m}, 27-$ 30.i.1992, Holzenthal, Muñoz, Kjer, 1 ơ, 1 \% (en alcohol).

\section{Leptonema vitum Flint, McAlpine y Ross} Fig. 12; Mapa 1.

Leptonema vitum Flint, McAlpine y Ross 1987: 58-59, figs. 554-560, ơ, Costa Rica: Puntarenas (NMNH); Holzenthal 1988: 68.

Diagnosis.-Adultos: Pequeños y con las alas translúcidas de color pardo. Ala anterior de 14 a $15 \mathrm{~mm}$ de longitud, y con una banda de pelillos pardo oscuros sobre el "cord".

Phallus (Fig. 12): Esta especie endémica puede ser reconocida de L. ekisi Flint, McAlpine y Ross, $L$. fortunum Flint, McAlpine y Ross, L. rafita Muñoz-Q. y L. salvini Mosely, por la forma y tamaño de los procesos " $e$ ", " $f$ " y " $g$ ", como se detalla en la diagnosis de $L$. rafita. Procesos " $c$ ", " $d$ ", " $f$ ' y " $j$ " ausentes en L. vitum.

Distribución: Costa Rica (Puntarenas).

Material examinado: Costa RicA: Puntarenas: Las Cruces, cerca de San Vito $\left[8.79^{\circ} \mathrm{N}, 82.97^{\circ} \mathrm{W}\right.$, el. $\left.1150 \mathrm{~m}\right], 24 . i v .1965$, S.S. \& W.D. Duckworth, ơ, holotipo (USNM).

\section{Leptonema woldianum Flint, McAlpine y Ross.}

Fig. 16; Mapa 2.

Leptonema woldianum Flint, McAlpine y Ross 1987: 59, figs. 561-568, ơ, Panamá: Chiriquí (NMNH); McElravy et al. 1981; 153; 1982: 307 (especie "B" de Leptonema no descrita); Holzenthal 1988: 68.

Diagnosis.-Adultos: Moderamente grandes y con las alas translúcidas de color pardo. Ala anterior de 18 a $21 \mathrm{~mm}$ de longitud, con un parche pequeño y redondeado de pelillos pardo oscuros sobre la vena tranversal " $m_{3}-m_{4}$ ". 
Phallus (Fig. 16): Esta especie está relacionada con $L$. sinuatum Mosely y $L$. turrialbum Flint, McAlpine y Ross, sin embargo difiere en la forma y tamaño de los procesos " $a$ ", " $b$ ", " $e$ " y " $f$ " como se detalla en las diagnosis de esas especies. Procesos " c", " $d$ " y " "j" ausentes en L. woldianum.

Distribución: COSTA Rica (Alajuela, Cartago, Guanacaste); PANAMÁ.

Material examinado: CosTA RICA: Alajuela: Río Toro, $3.0 \mathrm{~km}$ (road) SW Bajos del Toro, $10.204^{\circ} \mathrm{N}, 84.316^{\circ} \mathrm{W}$, el. $1530 \mathrm{~m}, 3-$ 4.ix.1990, Holzenthal,, Blahnik, Huisman, 1 O’, 1 \&; Queb. Virgencita, $10.2 \mathrm{~km} \mathrm{~S}$ Bajos del Toro, $10.168^{\circ} \mathrm{N}, 84.326^{\circ} \mathrm{W}$, el. $1780 \mathrm{~m}$, 10.ii.1992, Holzenthal, Muñoz, Kjer, $20^{\prime \prime}, 1$ \%; Cartago: Res. Tapantí, Queb. Segunda, @ administration building, $9.761^{\circ} \mathrm{N}, 83.787^{\circ} \mathrm{W}$, el. 1250 m, 9-10.v.1990, Holzenthal, Blahnik, 1 ơ, 1 \&; igual pero, Queb. Palmitos \& falls, $9.72^{\circ} \mathrm{N}, 83.78^{\circ} \mathrm{W}$, el. $1400 \mathrm{~m}, 24-25$.iii.1991, Holzenthal, Muñoz, Huisman, 2 ơ; Guanacaste: P. N. Guanacaste, Sta. Rosa, el. 300 m, iv.1985, D.H. Janzen, W. Hallwachs, 4 O’, 6 \%.

Leptonema asclepium Flint, McAlpine y Ross Fig. 20; Mapa 3.

Leptonema asclepium Flint, McAlpine y Ross 1987: 60, figs. 569-576, ơ, Costa Rica: Cartago (NMNH); Holzenthal 1988: 66.

Diagnosis.-Adultos: Pequeños y con las alas translúcidas de color pardo claro (en algunos especímenes, verde claro basalmente). Ala anterior de 14 a $16 \mathrm{~mm}$ de longitud, y con pelillos pardo claros sin ningún patrón particular.

Phallus (Fig. 20): Esta especie, endémica y cercana a $L$. campanum Flint, McAlpine y Ross (Fig. 21), L. championi Mosely, y L. dye$r i$ Flint, McAlpine y Ross, se puede reconocer por el tamaño del proceso " $b$ ". Proceso " $b$ ", en $L$. asclepium, en vista lateral, mucho más largo que el proceso " $c$ ", curvado ligeramente y dirigido anteroventralmente alcanzando el margen ventral del phallus; en L. campanum y L. championi, igual o ligeramente más largo que el proceso " $c$ " y dirigido posterodorsalmente; y en L. dyeri, más largo que el proceso " $c$ ", dirigido anteriormente y no alcanza el margen ventral del phallus. Proceso " $e$ ", en L. asclepium, con puntas minúsculas y superficiales; y en $L$. dyeri, éstas están ausentes. Esta especie difiere de L. simulans mayanum Flint, McAlpine y Ross, L. simulans simulans Mosely, y L. tica, nueva especie, por la forma de los procesos "b", "c", "g" y " "j", como se describe en las diagnosis de $L$. simulans simulans y $L$. tica. En L. asclepium, procesos " $d$ ", " $f$ ' ausentes.

Distribución: COSTA RICA (Alajuela, Cartago, Guanacaste, Limón, San José).

Material examinado: COSTA RICA: Alajuela: Cerro Campana, Río Bochinche trib., ca. $6 \mathrm{~km}$ (air) NW Dos Ríos, $10.945^{\circ} \mathrm{N}, 85.413^{\circ} \mathrm{W}$, el. 600 m, 22-23.vii.1987, Holzenthal, Morse, Clausen, 1 ơ, 1 (en alcohol); Guanacaste: Río Los Ahogados, $11.3 \mathrm{~km}$ ENE Quebrada Grande, $10.865^{\circ} \mathrm{N}, 85.423^{\circ} \mathrm{W}$, el. $470 \mathrm{~m}, 26 . v i .1986$, Holzenthal, Heyn, Armitage, 2 O'; P. N. Guanacaste, Est. Maritza, Río Tempisquito, $10.958^{\circ} \mathrm{N}$, 85.497 W, el. 550 m, 19-20.vii.1987, Holzenthal, Morse, Clausen, $10^{\prime 7}$ (en alcohol); igual pero, 1316.vii.1992, Muñoz, 1 O’, 3 \&; Est. Pitilla, Río Orosí, $10.991^{\circ} \mathrm{N}, 85.428^{\circ} \mathrm{W}$, el. $700 \mathrm{~m}, 22-$ 25.v.1990, Holzenthal, Blahnik, 4 O'; Z. P. Tenorio, tribs. to Río San Lorenzo, $6 \mathrm{~km}$ NW Tierras Morenas, $10.61^{\circ} \mathrm{N}, 84.98^{\circ} \mathrm{W}$, el. $900 \mathrm{~m}, 17-$ 19.ii.1992, Holzenthal, Muñoz, Kjer, 10'; Limón: P. N. Braulio Carrillo, Queb. González, $10.160^{\circ} \mathrm{N}$, 83.939 W, el. 480 m, 12-14.v.1990, Holzenthal, Blahnik, 6 ơ, 9 ( 2 ơ, 3 \& en alcohol).

\section{Leptonema campanum Flint, McAlpine y Ross}

Fig. 21; Mapa 3.

Leptonema campanum Flint, McAlpine y Ross 1987: 60, figs. 577-583, Ơ, Panamá: Panamá (NMNH); Holzenthal 1988: 66.

Diagnosis.-Adultos: Pequeños y con las alas translúcidas de color pardo claro (en algunos especímenes, verde claro basalmente). Ala anterior de 14 a $17 \mathrm{~mm}$ de longitud, y con una banda de pelillos pardo oscuros sobre el "cord".

Phallus (Fig. 21): Esta especie se distingue de L. championi Mosely, y L. dyeri Flint, 


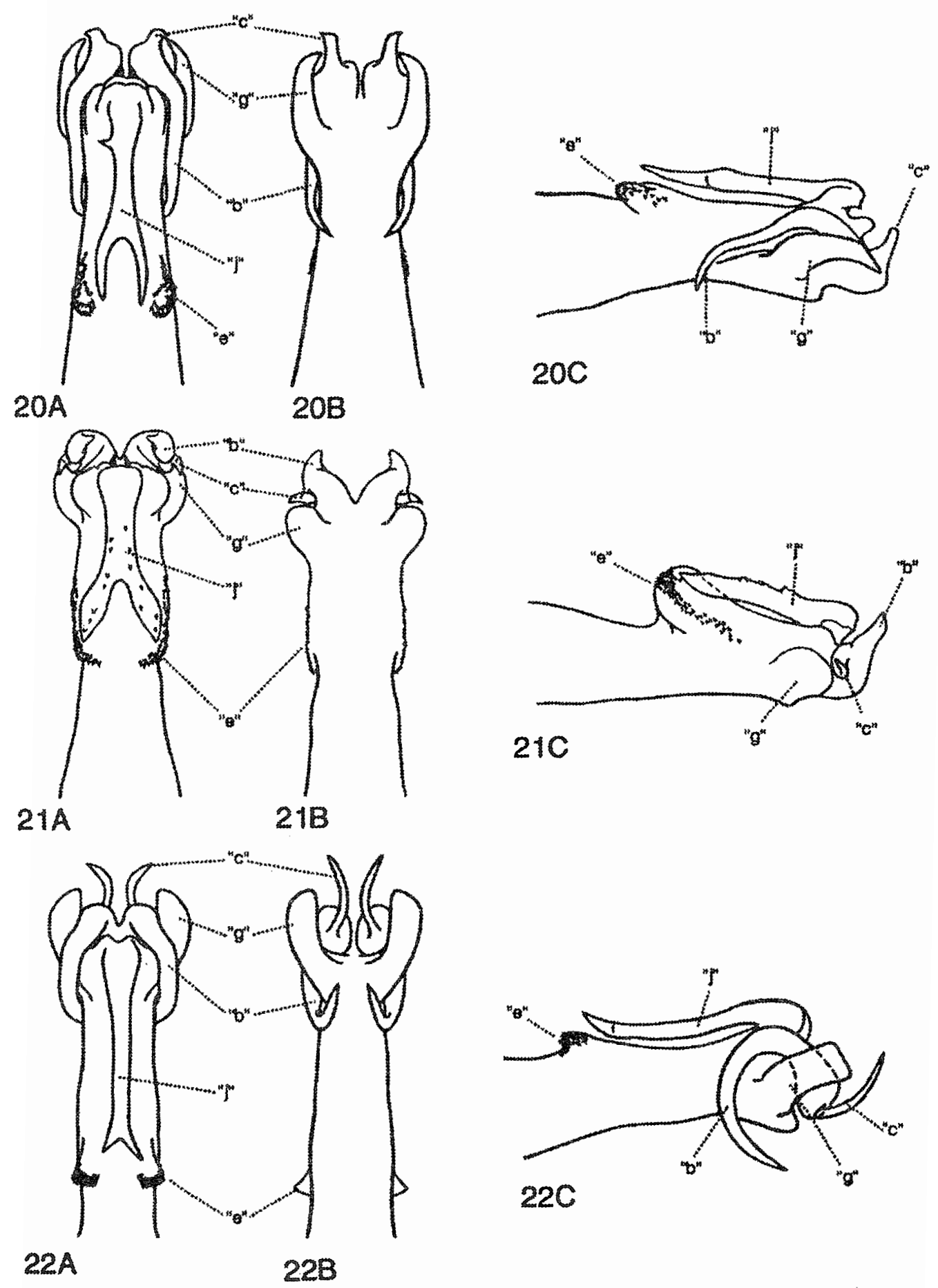

Figs. 20-22. Phallus: 20. L. asclepium, 21. L. campanum, 22. L. simulans simulans. -A., vista dorsal; B., vista ventrall; C., vista lateral. 
McAlpine y Ross, por la forma y tamaño del proceso " $g$ ". Proceso " $g$ ", en L. campanum, en vista ventral, corto, ventrolateral y con el ápice redondeado alcanzando la base de los procesos " $b$ " y "c"; en $L$. championi, en vista ventral, con una proyección apicolateral, puntiaguda y dirigida mesialmente; y en $L$. dyeri, en vista ventral, semeja un gancho alargado que alcanza el margen posterior del proceso " $b$ ". Esta especie difiere de L. asclepium Flint, McAlpine y Ross, L. simulans simulans Mosely, y L. tica, nueva especie, por la forma y tamaño de los procesos " $b$ ", " $c$ " y " $g$ ", como se menciona en las diagnosis de esas especies. Procesos " $d$ " y " $f$ " ausentes en L. campanum.

Distribución: Costa Rica (Alajuela, Puntarenas, San José); PANAMÁ.

Material examinado: Costa RicA: Alajuela: Cerro Campana, ca. $6 \mathrm{~km}$ (air) NW Dos Ríos, $10.9^{\circ} \mathrm{N}, 85.4^{\circ} \mathrm{W}$, el. $640 \mathrm{~m}$, 15-16.iii.1986, Holzenthal, Fasth, 1 ơ; Res. Forestal San Ramón, Río San Lorencito \& tribs., $10.216^{\circ} \mathrm{N}, 84.607^{\circ} \mathrm{W}$, el. $980 \mathrm{~m}, 2$ 4.vii.1986, Holzenthal, Heyn, Armitage, 16 O’, 19 \&; igual pero, 2-6.ix.1986, I. \& A. Chacón, 1 đ"; 24-27.ii.1987, 1 đ’, 3 ᄋ; 30.iii.-1.iv.1987, Holzenthal, Hamilton, Heyn, 21 O’ (1 en alcohol), 18 \%; 1-4.v.1990, Holzenthal, Blahnik, $70^{\circ}, 40$ \& $\left(10^{\prime \prime}, 9\right.$ \& en alcohol); 28-30.vii.1990, Holzenthal, Blahnik, Muñoz, 1 ơ, 9 \&; 6-10.iii.1991, Holzenthal, Muñoz, Huisman, 11 ơ, 21 \%; Puntarenas: Río Jaba, $2.4 \mathrm{~km}$ (air) NW San Vito, $8.832^{\circ} \mathrm{N}, \quad 82.991^{\circ} \mathrm{W}$, el. $970 \mathrm{~m}$, 13.vi.1986, Holzenthal, Heyn, Armitage, 1 o", 1 \&; Río Jaba, at rock quarry $1.4 \mathrm{~km}$ (air) W Las Cruces, $8.79^{\circ} \mathrm{N}, 82.97^{\circ} \mathrm{W}$, el. $1150 \mathrm{~m}$, 9.viii.1990, Holzenthal, Blahnik, Muñoz, 4 ơ, 2 \&; Las Cruces, Jardín Botánico, R. \& C. Wilson, $8.79^{\circ} \mathrm{N}, 82.97^{\circ} \mathrm{W}$, el. $1200 \mathrm{~m}$, 15.iv.1989, Holzenthal, Blahnik, 1 ơ; San José: P. N. Braulio Carrillo, Queb. Sanguijuela, Est. Carrillo, $10.160^{\circ} \mathrm{N}, 83.963^{\circ} \mathrm{W}$, el. 800 m, 27.iii.1987, Holzenthal, Hamilton, Heyn, 10 o".

\section{Leptonema simulans simulans Mosely}

Fig. 22; Mapa 3.

Leptonema simulans simulans Mosely 1933: 32-34, figs. 74-80, ơ, Panamá: Chiriquí (BMNH); Fischer 1963: 172; 1972: 156; Weidner 1964: 84; McElravy et al. 1981: 153; 1982: 307, 310, 312; Flint, McAlpine y Ross 1987: 62, figs. 600-607; Holzenthal 1988: 67.

Diagnosis.-Adultos: Moderamente grandes y con las alas translúcidas de color pardo claro a verde claro. Ala anterior de 18 a $21 \mathrm{~mm}$ de longitud, y con una banda tenue de pelillos pardo oscuros sobre el "cord".

Phallus (Fig. 22): Esta especie es ampliamente distribuida en el país y similar a $L$. asclepium Flint, McApine y Ross (Fig. 20) y $L$. campanum Flint, McAlpine y Ross (Fig. 21), pero reconocible por la forma de los procesos "b", "c", "g" у "j". Proceso "b", en L. s. simulans, más largo que el proceso " $c$ ", en vista lateral, fuertemente curvado basalmente, casi circular y con el ápice proyectado posteroventralmente sobrepasando el margen ventral del phallus; en L. asclepium, más largo que el proceso " $c$ ", en vista lateral, arqueado levemente y proyectado anteroventralmente alcanzando el margen ventral del phallus; y en L. campanum, corto, en vista lateral, subtriangular y dirigido posterodorsalmente. Proceso " $c$ ", en esta especie, en vista lateral, emerge en el margen ventrobasal del proceso " $b$ ", delgado, curvado, puntiagudo y dirigido dorsalmente; en $L$. asclepium, corto y robusto, en vista lateral, emerge en el margen posterobasal del proceso " $b$ ", subtriangular y dirigido posterodorsalmente, en vista ventral, erguido, con el ápice curvado y dirigido lateralmente; y en $L$. campanum, corto, en vista lateral, emerge en el margen laterobasal del proceso " $b$ ", y dirigido lateralmente. Proceso " $g$ ", en L. s. simulans, un lóbulo lateroapical, en vista lateral, alargado y subcuadrado, en vista ventral, semeja un gancho con el ápice redondeado levemente que sobrepasa la base de los procesos " $b$ " y " $c$ "; en $L$. asclepium, un lóbulo lateroapical, en vista lateral, alargado y subtriangular, en vista ventral, 
semeja un gancho con el ápice puntiagudo y dirigido mesialmente que sobrepasa la base de los procesos " $b$ " y " $c$ "; y en L. campanum, un lóbulo lateroventral, en vista ventral, corto, globular con el ápice redondeado y apenas alcanza la base de los procesos " $b$ " y " $c$ ". Proceso " $j$ ”, en L. asclepium, con una o más proyecciones laterales pequeñas; en $L$. s. simulans y L. s. mayanum Flint, McAlpine y Ross, sin proyecciones laterales. El tamaño de la bifurcación apical del proceso “ $j$ ”, es el único rasgo que nos permite separar a $L$. s. simulans de $L$. s. mayanum. La bifurcación apical del proceso “j”, en L. s. simulans, en vista dorsal, leve, a lo sumo alcanza 1/4 del largo del proceso " $j$ ”; en L. s. mayanum y L. asclepium, más grande, alcanza más de 1/3 del largo del proceso " $j$ ". Esta especie difiere de L. tica, nueva especie, por la forma y tamaño de los procesos " $b$ " y " $c$ ", como se detalla en la diagnosis de esa especie. Procesos " $d$ " y " $f$ " ausentes en $L$. s. simulans.

Distribución: CosTA Rica (Alajuela, Cartago, Guanacaste, Puntarenas, San José); PANAMÁ.

Material examinado: Costa RICA: Alajuela: Río La Paz Pequeña, route 9, $7.8 \mathrm{~km} \mathrm{~N}$ Vara Blanca, $10.211^{\circ} \mathrm{N}, 84.166^{\circ} \mathrm{W}$, el. $1230 \mathrm{~m}$, 13.ii.1986, Holzenthal, Morse, Fasth, 1 ơ; Río Sarapiquí, ca. $2 \mathrm{~km}$ SE Cariblanco, $10.299^{\circ} \mathrm{N}$, $84.172^{\circ} \mathrm{W}$, el. $710 \mathrm{~m}, 6 . i i .1986$, I. \& A. Chacón, 3 ơ, 5 \&; Cerro Campana, Río Bochinche trib., ca. 6 km (air) NW Dos Ríos, $10.945^{\circ} \mathrm{N}$, $85.413^{\circ} \mathrm{W}$, el. $600 \mathrm{~m}, 22-23 . v i i .1987$, Holzenthal, Morse, Clausen, 1 O'; Res. Forestal San Ramón, Río San Lorencito \& tribs., $10.216^{\circ} \mathrm{N}$, $84.607^{\circ} \mathrm{W}$, el. $980 \mathrm{~m}, 2-6 . i x .1986$, I. \& A. Chacón, 1 ơ; igual pero, 24-27.ii.1987, 1 ơ, 4 \%; 30.iii.-1.iv.1987, Holzenthal, Hamilton, Heyn, 2 ơ; 1-4.v.1990, Holzenthal, Blahnik, 5 ơ (3 en alcohol), 2 \&; 6-10.iii.1991, Holzenthal, Muñoz, Huisman, 2 o', 2 \&; Queb. Virgencita, $10.2 \mathrm{~km} S$ Bajos del Toro, $10.168^{\circ} \mathrm{N}$, $84.326^{\circ} \mathrm{W}$, el. $1780 \mathrm{~m}, 5-6.1 x .1990$, Holzenthal, Blahnik, Huisman, 3 o', 5 \&; igual pero, 10.ii.1992, Holzenthal, Muñoz, Kjer, 2 ơ, 3 \&; Queb. Latas, $8.9 \mathrm{~km}$ NE Bajos del Toro, $10.269^{\circ} \mathrm{N}, 84.260^{\circ} \mathrm{W}$, el. $1030 \mathrm{~m}$, 6.ix.1990, Holzenthal, Blahnik, Huisman, 1 ơ; Río Toro,
$3.0 \mathrm{~km}$ (road) SW Bajos del Toro, $10.204^{\circ} \mathrm{N}$, $84.316^{\circ} \mathrm{W}$, el. $1530 \mathrm{~m}, 3-4.1 x .1990$, Holzenthal, Blahnik, Huisman, 4 ơ, 3 \%; igual pero, 11.ii.1992, Holzenthal, Muñoz, Kjer, 2 O' (1 en alcohol), 1 \%; Río Peje \& falls, ca. $1 \mathrm{~km} \mathrm{SE}$ San Vicente, $10.277^{\circ} \mathrm{N}, 84.388^{\circ} \mathrm{W}$, el. $1450 \mathrm{~m}$,

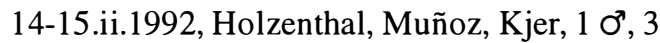
\%; Cartago: Res. Tapantí, Queb. Segunda, @ administration building, $9.761^{\circ} \mathrm{N}, 83.787^{\circ} \mathrm{W}$, el. 1250 m, 9-10.v.1990, Holzenthal, Blahnik, $70^{\circ}, 8$ \& ( $10^{\circ}, 3$ \& en alcohol); Guanacaste: P. N. Rincón de la Vieja, Río Negro, $10.765^{\circ} \mathrm{N}$, $85.313^{\circ} \mathrm{W}$, el. $810 \mathrm{~m}$, 3.iii.1986, Holzenthal, Fasth, 16 ơ, 13 \%; igual pero, Queb. Zopilote, $10.765^{\circ} \mathrm{N}, 85.309^{\circ} \mathrm{W}$, el. $785 \mathrm{~m}$, 3.iii.1986, Holzenthal, 16 ơ, 10 \% (en alcohol); P. N. Guanacaste, Río San Josecito, Est. Mengo [Est. Cacao], $10.922^{\circ} \mathrm{N}, 85.470^{\circ} \mathrm{W}$, el. $960 \mathrm{~m}, 3-$ 4.iv.1987, Holzenthal, Hamilton, Heyn, 13 ơ, 24 ९; igual pero, 28-29.vii.1987, Holzenthal, Morse, Clausen, 7 ơ, 24 \& (1 ơ, 8 \& en alcohol); Est. Maritza, Río Tempisquito, $10.958^{\circ} \mathrm{N}$, $85.497^{\circ} \mathrm{W}$, el. $550 \mathrm{~m}, 19-20$.vii.1987," Holzenthal, Morse, Clausen, 10 ơ $^{\prime}(1$ en alcohol); igual pero, 13-16.vii.1992, Muñoz, 1 ơ, 2 \&; Río Tempisquito Sur, $10.95^{\circ} \mathrm{N}, 85.48^{\circ} \mathrm{W}$, el. $600 \mathrm{~m}$, 30.viii.1990, Huisman, Quesada, 3 ơ, 2 9 (en alcohol); Est. Pitilla, Río Orosí, $10.991^{\circ} \mathrm{N}$, $85.428^{\circ} \mathrm{W}$, el. $700 \mathrm{~m}, 22-25 . v .1990$, Holzenthal, Blahnik, 1 O'; igual pero, 2.vi.1986, D.H. Janzen, 4 ơ, 5 \& (en alzohol); Z. P. Tenorio, tribs. to Río San Lorenzo, $6 \mathrm{~km}$ NW Tierras Morenas, $10.61^{\circ} \mathrm{N}, 84.98^{\circ} \mathrm{W}$, el. $900 \mathrm{~m}, 17-$ 19.ii.1992, Holzenthal, Muñoz, Kjer, 1 ○’, 1 \%; Puntarenas: Río Bellavista, ca. $1.5 \mathrm{~km}$ NW Las Alturas, $8.951^{\circ} \mathrm{N}, 82.846^{\circ} \mathrm{W}$, el. $1400 \mathrm{~m}, 2-$ 3.viii.1987, Holzenthal, Morse, Clausen, $10^{\circ}$; igual pero, 10-11.viii.1990, Holzenthal, Blahnik, Muñoz, 1 ơ, 1 \&; 16-17.iii.1991, Holzenthal, Muñoz, Huisman, 1 ơ; trib. to Río Bellavista in Las Alturas (road to quarry), $8.952^{\circ} \mathrm{N}$, $82.848^{\circ} \mathrm{W}$, el. $1480 \mathrm{~m}, 13-14$.viii.1990, Holzenthal, Blahnik, Muñoz, 5 ơ, 4 \& (1 O', 1 \% en alcohol); igual pero, 19.iii.1991, Holzenthal, Muñoz, Huisman, 3 ơ; Z. P. Las Tablas, Río Cotón, Sitio Cotón, $8.941^{\circ} \mathrm{N}, 82.787^{\circ} \mathrm{W}$, el. 1460 m, 15.iv.1989, Holzenthal, Blahnik, 2 ơ, 4 \&; small spring seep to Río Cotón in Las Al- 
turas, $8.938^{\circ} \mathrm{N}, 82.824^{\circ} \mathrm{W}$, el. $1380 \mathrm{~m}$, 14.viii.1990, Holzenthal, Blahnik, Muñoz, $10^{\prime \prime}$ (en alcohol); Río Cotón, in Las Alturas, $8.938^{\circ} \mathrm{N}, 82.826^{\circ} \mathrm{W}$, el. $1360 \mathrm{~m}, 12$.viii.1990, Holzenthal, Blahnik, Muñoz, 1 ơ, 2 \& (en alcohol); San José: Escazú, el. 1300 m, 2027.v.1987, H. \& M. Townes, 2 ơ, 3 \& (en alcohol); P. N. Braulio Carrillo, Zurquí, Moravia, el. 1600 m, xi.1988, P. Hanson, 1 o’, 1 ㅇ (en alcohol); Río Parrita Chiquito, rt. 12, 6.5 km SW jct. rt. $2,9.703^{\circ} \mathrm{N}, 83.970^{\circ} \mathrm{W}$, el. $1990 \mathrm{~m}$, 18.vi.1986, Holzenthal, Heyn, Armitage, 2 o', 3 \&; igual pero, 10.iv.1987, Holzenthal,Hamilton, Heyn, 7 ơ, 4 \&; El Salvaje, Río Tabarcia, $8 \mathrm{~km}$ (rd.) E Palmichal, 9.847 ${ }^{\circ} \mathrm{N}, 84.164^{\circ} \mathrm{W}$, el. 1650 m, 19-21.i.1992, Holzenthal, Kjer, Quesada, 4 ơ, 6 of; Queb. Caraigres, 2.5 km (rd) SW La Legua, $9.734^{\circ} \mathrm{N}, 84.120^{\circ} \mathrm{W}$, el. $1470 \mathrm{~m}$, 22.i.1992, Holzenthal, Kjer, Quesada, $10^{7}$.

\section{Leptonema tica Muñoz-Q., New Species}

Fig. 23; Map 3.

Diagnosis: This species belongs within the simulans Group, as defined by Flint et al. (1987). It is similar to L. asclepium Flint, McAlpine, and Ross (Fig. 20), L. campanum Flint, McAlpine and Ross (Fig. 21), and L. s. simulans Mosely (Fig. 22) differing from those species by the shape of process " $b$ " and the absence of process " $c$ " of the phallus. Process " $\boldsymbol{b}$ ", in the new species arises ventromesally, is short, directed dorsally, and bifurcated apically; in L. asclepium, it arises apicomesally, is long, slender, arched, projected anteroventrally beyond the base of process " $g$ ", and unforked; in L. campanum, it arises apicolaierally, is short, directed posterodorsally, and unforked; and in L. s. simulans, it arises apicomesally, is short, slender, directed posterodorsally, and unforked. Process " $c$ ", in L. tica, is absent; in L. asclepium, L. campanum, and L. s. simulans, it is present.

Description.-Male: Length of forewing $16 \mathrm{~mm}$. Body sclerites pale yellow. Dorsum of head pale yellow, with short, light brown setae. Legs with fine, yellowish setae. Wings pale yellow, translucent; forewing covered with fine, short, yellowish setae. Maxillary palpus with fifth segment about 2/3 length of basal 4 segments combined. Process of sternum V large, oval. Genitalia: (Fig. 23) Segment IX, as viewed laterally, narrow, elongate; as viewed dorsally, with V-shaped dorsal keel. Segment X with warts " $a$ ", " $b-1$ ", and " $b-2$ " occurring close together, each elongate, their bases narrow, apices bulbous, with small setae; wart " $c$ " absent; lateral lobes, as viewed dorsally, with lateral margin slightly sinuous, bearing short setae, apex pointed, projecting posteriorly. Inferior appendage two segmented, basal segment less than 3 times length of apical segment; apical segment with short setae on inner margin. Phallus with midsection long, tubular; apical section bearing two, triangular, sharply pointed, phallotremal sclerites behind process " $a$ ", visible in dorsal view; process " $a$ " visible in dorsal view, membranous, arising dorsomesally, subtriangular, broad, apex rounded, slightly narrower than base of process; process " $b$ " lightly sclerotized, arising ventromesally; as viewed laterally, short, stout, directed dorsally, bifurcated apically, with apices subtriangular, slender, sharply pointed; as viewed dorsally, process " $b$ " directed laterally, sinuous, bifurcated; posterior apex curved, hooklike; anterior apex short, subtriangular, pointed; as viewed ventrally, apical margin of process " $b$ " sinuous, emarginate apicomesally; process " $e$ " dorsolateral, fused to midsection of phallus; as viewed dorsally, developed into two elongate rows of spicules, ending in rounded, membranous, dorsal and anteriorly directed lobes, with small spicules; process " $g$ " subapicolateral; as viewed laterally, stout, broad, curved at middle, projecting dorsoposteriorly, apex sharply pointed, reaching apical margin of process " $b$ "; as viewed dorsally and ventrally, lateral margin of process " $g$ " convex; internal margin of process " $g$ " slightly concave, apex pointed; process "6 $j$ " lightly sclerotized, tubular, elongate, stout; as viewed dorsally, arising dorsomesally above phallotremal sclerites and at base of process 

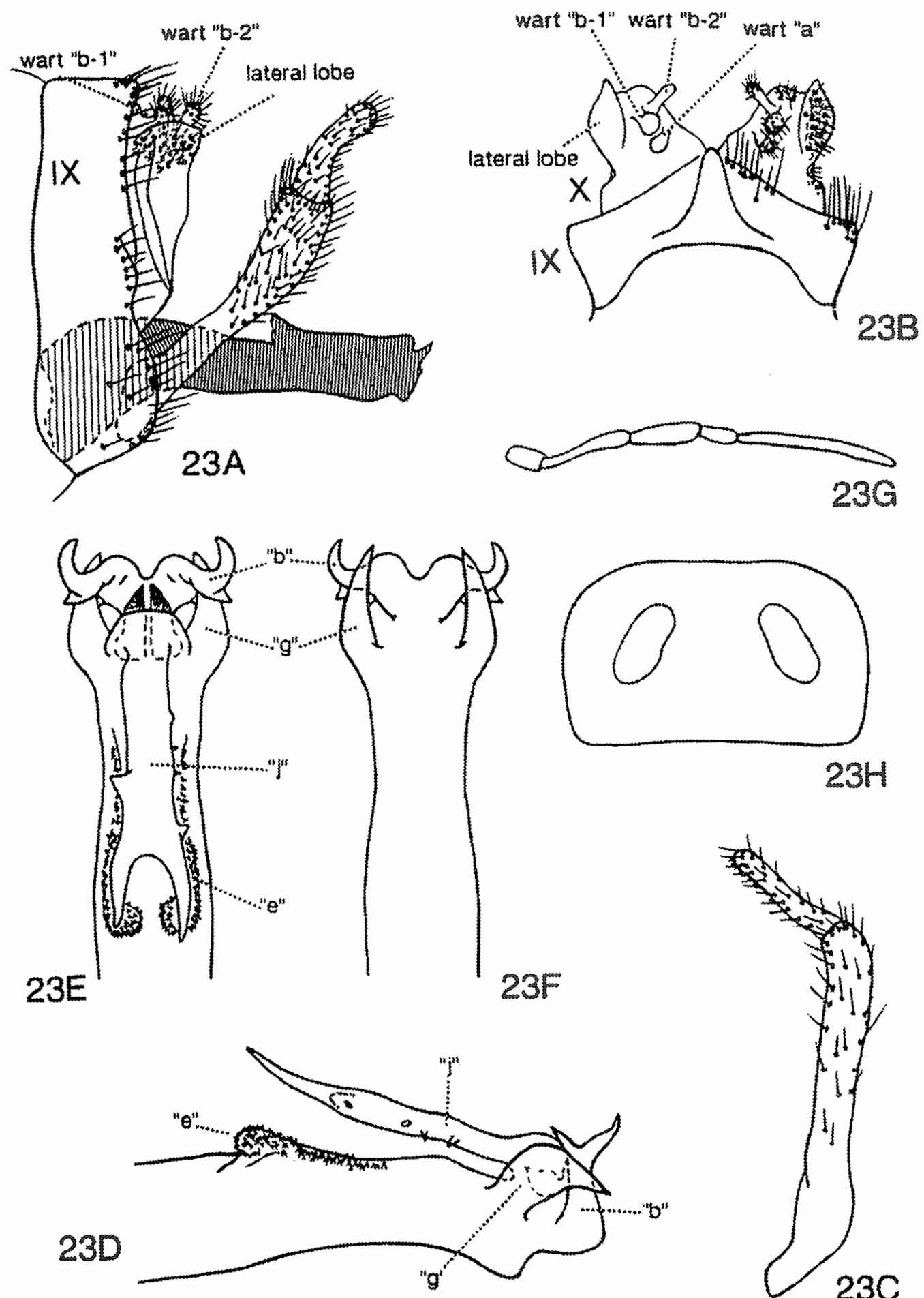

Fig. 23. Leptonema tica, new species: A., male genitalia, lateral view; B., segments IX, X, dorsal view; E., phallus, dorsal view; F. phallus, ventral view; G., maxillary palpus, lateral view; H., sternum V, ventral view. 
" $a$ ", projected anteriorly, reaching dorsal lobes of process " $e$ "; with several, stout, spinelike, lateral projections; with conspicuous, apical bifurcation, being $1 / 3$ total length of process " $j$ "; apical projections slender, slightly curved, pointed; processes " $c$ ", " $d$ ", and " $\rho$ ' absent.

Type material: HOLOTYPE: $\sigma^{\prime}$, COSTA RICA: San José: Parque Nacional Braulio Carrillo: Estación Carrillo, Quebrada Sanguijuela, $10.160^{\circ} \mathrm{N}, 83.963^{\circ} \mathrm{W}$, el. $800 \mathrm{~m}, 25 . \mathrm{v} .1992$, F. Muñoz Q. (NMNH).

Etymology: Dedicated to Costa Rican women, who are known as "Ticas".

Diagnosis.-Adultos: Pequeños y con las alas translúcidas de color amarillo pálido. Ala anterior de $16 \mathrm{~mm}$ de longitud, y cubierta de pelillos amarillos.

Phallus (Fig. 23): Esta nueva especie difiere de L. asclepium Flint, McAlpine y Ross (Fig. 20), L. campanum Flint, McAlpine y Ross (Fig. 21) y L. s. simulans Mosely (Fig. 22 ), por la forma del proceso " $b$ " y la presencia del proceso " $c$ ". Proceso " $b$ ", en L. tica, apical, emerge mesoventralmente, en vista lateral, corto, robusto, dirigido dorsalmente y bifurcado apicalmente; en L. asclepium, emerge mesoapicalmente, en vista lateral, largo, delgado, arqueado, dirigido anteroventralmente y no bifurcado; en L. campanum, emerge lateroapicalmente, en vista lateral, corto, subtriangular, dirigido posterodorsalmente y no bifurcado; y en L. s. simulans, emerge mesoapicalmente, en vista lateral, largo, delgado, fuertemente curvado, casi circular, dirigido posteroventralmente y no bifurcado. Proceso " $c$ ", en $L$. tica, ausente; y en las otras especies, presente. En $L$. tica, procesos " $c$ ", " $d$ " y " $f$ ", ausentes.

\section{Leptonema albovirens (Walker)}

Fig. 13; Mapa 3.

Leptonema albovirens (Walker) 1852: 76, lectotipo ơ, Venezuela (Macronema) (BMNH); Banks 1914: 150; Mosely 1933: 45, figs. 123-130; Betten y Mosely 1940: 188, fig. 93 (0'); Fischer 1963: 166; Flint 1967: 8; 1968 : 31, 32; 1981: 19; Bueno-Soria y Flint 1978:
208-209; Harrison y Rankin 1976: 275-311; Flint, McAlpine y Ross 1987: 65, figs. 1, 4-6, 13, 637-646; Holzenthal 1988: 66.

Leptonema guatemalum Banks 1913: 89, Pl. IV: figs. 9-11, ơ; Flint 1967: 8 (sinonimia).

Diagnosis.-Adultos: Pequeños y con las alas translúcidas de color pardo claro a verde claro. Ala anterior de 12 a $17 \mathrm{~mm}$ de longitud, y cubierta tenuamente con pelillos amarillos.

Phallus (Fig. 13): Esta especie ha sido la más abundante en las recolectas examinadas y más ampliamente dispersa en el país. Es la única especie del Grupo pallidum presente en Costa Rica, y aunque semejante a $L$. moselyi Flint, McAlpine y Ross, L. ramosum Flint, McAlpine y Ross, y L. viridianum Návas, se reconoce por la forma y tamaño de los procesos " $a$ " y " $d$ ". Proceso " $a$ ", en L. albovirens y L. moselyi, en vista lateral, robusto, corto, truncado y archeado sin proyecciones o crestas dorsoapicales; en L. ramosum y L. viridianum, con proyecciones o crestas dorsoapicales. Proceso " $d$ ", en $L$. albovirens, en vista lateral, con numerosas espinas pequeñas, la base curvada fuertemente, largo y dirigido posteroventralmente sobrepasando el margen ventral del phallus; en L. moselyi, corto, curvado levemente y dirigido posteriormente; en $L$. ramosum y $L$. viridianum, sin espinas pequeñas, curvado ligeramente, dirigido posteroventralmente $\mathrm{y}$ corto, apenas alcanza el margen ventral del phallus. En L. albovirens, procesos " $\rho$ ", ."g" y "j" ausentes.

Distribución: EUA (Texas); MÉXICO; BElice; Guatemala; Honduras; Nicaragua; Costa Rica (Alajuela, Cartago, Guanacaste, Heredia, Limón, Puntarenas, San José); PanAmá; Colombia; Venezuela; Trinidad y TobaGO; GRANADA; SAN Vicente.

Material examinado: CostA RICA: Alajuela: Río Pizote, ca. $5 \mathrm{~km} \mathrm{~N}$ Dos Ríos, $10.948^{\circ} \mathrm{N}, 85.291^{\circ} \mathrm{W}$, el. $470 \mathrm{~m}$, 9.iii.1986, Holzenthal, Fasth, 21 ơ $^{\prime}, 29$ (5 ơ, 12 en alcohol); Río Pizote, ca. 5 km (air) S. Brasilia, $10.972^{\circ} \mathrm{N}, 85.345^{\circ} \mathrm{W}$, el. $390 \mathrm{~m}, 12 . i i i .1986$, Holzenthal, Heyn, Fasth, $360^{\prime}, 55$ \& (13 0', 41 Q en alcohol); Cerro Campana, ca. 6 km (air) . NW Dos Ríos, $10.9^{\circ} \mathrm{N}, 85.4^{\circ} \mathrm{W}$, el. $640 \mathrm{~m}, 15$ - 
16.iii.1986, Holzenthal, Fasth, 3 ơ; Cerro Campana, Río Bochinche trib., ca. 6 km (air) NW Dos Ríos, $10.945^{\circ} \mathrm{N}, 85.413^{\circ} \mathrm{W}$, el. $600 \mathrm{~m}$, 22-23.vii.1987, Holzenthal, Morse, Clausen, 1 o'; Res. Forestal San Ramón, Río San Lorencito \& tribs., $10.216^{\circ} \mathrm{N}, 84.607^{\circ} \mathrm{W}$, el. $980 \mathrm{~m}, 2-$ 4.vii.1986, Holzenthal, Heyn, Armitage, 3 ơ; igual pero, 5-9.vii.1986, I. \& A. Chacón, 1 O’; 2-6.ix.1986, 3 o"; 1-4.x.1986, 4 ơ, 2 \%; 2427.ii.1987, 4 ơ; 30.iii.-1.iv.1987, Holzenthal, Hamilton, Heyn, 11 ơ (4 en alcohol); 2830.vii.1990, Holzenthal, Blahnik, Muñoz, 1 ơ, 5 \&; Cartago: Río Platanillo, 2.2 km E Tayutic, $9.82^{\circ} \mathrm{N}, 83.55^{\circ} \mathrm{W}$, el. $730 \mathrm{~m}, 30 . i .1986$, Holzenthal, Morse, Fasth, $120^{\circ}, 8$ \% (5 ơ, 2 \% en alcohol); Queb. Platanillo, ca $5 \mathrm{~km}$ E Moravia de Chirripó, $9.821^{\circ} \mathrm{N}, 83.407^{\circ} \mathrm{W}$, el. 1130 m, 6.viii.1987, Holzenthal, Morse, Clausen, 3 ơ, 1 \%; Río Reventazón in CATIE [Turrialba], along Sendero Espaveles, $9.893^{\circ} \mathrm{N}, 83.651^{\circ} \mathrm{W}$, el. 500 m, 22.iii.1991, Fdo. Muñoz-Q., 13 ơ, 21 \&; Guanacaste: Río Tempisquito, ca. $3 \mathrm{~km}$ $\mathrm{S}$ route $1,10.790^{\circ} \mathrm{N}, 85.552^{\circ} \mathrm{W}$, el. $75 \mathrm{~m}$, 6.iii.1986, Holzenthal, Fasth, 7 ơ, 6 ९ (5 ơ, 3 ○ en alcohol); Queb. García, $10.6 \mathrm{~km}$ ENE Quebrada Grande, $10.862^{\circ} \mathrm{N}, 85.428^{\circ} \mathrm{W}$, el. 470 m, 8.iii.1986, Holzenthal, Fasth, 11 ơ; Río Mena, $4.2 \mathrm{~km} \mathrm{~W} \mathrm{Sta.} \mathrm{Cecilia,} 11.059^{\circ} \mathrm{N}$, 85.448 $\mathrm{W}$, el. $260 \mathrm{~m}, 11$. iii.1986, Holzenthal, Fasth, 23 ơ, 14 \& (12 ơ, 11 \& en alcohol); Río Los Ahogados, 11.3 km ENE Quebrada Grande, $10.865^{\circ} \mathrm{N}, 85.423^{\circ} \mathrm{W}$, el. $470 \mathrm{~m}, 7 . i i i .1986$, Holzenthal, Fasth, 3 ơ (1 0', 3 \& en alcohol); igual pero, 26.vi.1986, Holzenthal, Heyn, Armitage, 18 ơ, 16 \%; Río Tizate, 7.2 km NE Cañas Dulces, $10.773^{\circ} \mathrm{N}, 85.449^{\circ} \mathrm{W}$, el. $275 \mathrm{~m}$, 28.vi.1986, Holzenthal, Heyn, Armitage, 37 ơ, 15 \& (6 ơ, 9 \& en alcohol); Río Góngora, (sulfur mine) $4 \mathrm{~km}$ (air) NE Quebrada Grande, $10.887^{\circ} \mathrm{N}, 85.470^{\circ} \mathrm{W}$, el. $590 \mathrm{~m}, 21 . v i i .1987$, Holzenthal, Morse, Clausen, 67 ơ, 53 \& (21 ơ, 18 \& en alcohol); P. N. Guanacaste, El Hacha, Queb. Alcornoque, $11.009^{\circ} \mathrm{N}, 85.577^{\circ} \mathrm{W}$, el. 250 m, 26.vii.1987, Holzenthal, Morse, Clausen, 5 ơ, 11 \& (3 O’, 4 \& en alcohol); El Hacha, Queb. Pedregal, $10.983^{\circ} \mathrm{N}, 85.539^{\circ} \mathrm{W}$, el. 300 m, 27.vii.1987, Holzenthal, Morse, Clausen, 1 ơ, 2 \&; Queb. El Duende, nr La Casona, $10.838^{\circ} \mathrm{N}, 85.614^{\circ} \mathrm{W}$, el. $280 \mathrm{~m}, 29 . v i .1986$, Holzenthal, Heyn, Armitage, 1 ơ; Río Tempisquito, Hda Tempisquito (Pelón de la Altura), 1 $\mathrm{km} \mathrm{NE} \mathrm{km} \mathrm{265,} \mathrm{rt} 1,10.84^{\circ} \mathrm{N}, 85.56^{\circ} \mathrm{W}$, el. 100 m, 18.vii.1987, Holzenthal, Morse, Clausen, $180^{\circ}, 8$ \& (5 0 $0^{\circ}, 1$ \% en alcohol); igual pero, $10.847^{\circ} \mathrm{N}, 85.561^{\circ} \mathrm{W}$, el. $95 \mathrm{~m}, 6 . v i .1988$, E. de la Rosa, 4 O’, 12 \%; P. N. Guanacaste, Est. Pitilla, Río Orosí, $10.991^{\circ} \mathrm{N}, 85.428^{\circ} \mathrm{W}$, el. $700 \mathrm{~m}$, 22-25.v.1990, Holzenthal, Blahnik, 12 ơ, 6 \%; Est. Maritza, Río Tempisquito, $10.958^{\circ} \mathrm{N}$, $85.497^{\circ} \mathrm{W}$, el. $550 \mathrm{~m}, 19-20 . v i i .1987$, Holzenthal, Morse, Clausen, 1 ơ, 9 \%; igual pero, 30 31.viii.1990, Huisman, Blahnik, Quesada; 16 ơ (7 en alcohol), 18 \%; ca. 0.7 km N Est. Maritza, $10.96^{\circ} \mathrm{N}, 85.50^{\circ} \mathrm{W}$, el. $550 \mathrm{~m}$, 31.viii. 90 , Huisman, Quesada, 3 ơ; Heredia: Río Bijagual, on road to Magsasay, $10.408^{\circ} \mathrm{N}$, $84.076^{\circ} \mathrm{W}$, el. $140 \mathrm{~m}, 12 . i i .1986$, Holzenthal, Morse, Fasth, 7 o', 6 \& (3 ơ, 1 \% en alcohol); Río Sarapiquí, $7 \mathrm{~km} \mathrm{~W}$ Pto Viejo, $10.452^{\circ} \mathrm{N}$, $84.067^{\circ} \mathrm{W}$, el. $50 \mathrm{~m}, 11$. ii.1986, Holzenthal, Morse, Fasth, 6 ơ, 12 (4 ơ, 5 en alcohol); Est. Biol. La Selva, Queb. Sura, $10.437^{\circ} \mathrm{N}$, $84.010^{\circ} \mathrm{W}$, el. $50 \mathrm{~m}, 20-21 . v i .1986$, Holzenthal, Heyn, Armitage, 29 ơ, 12 (3 ơ, 1 \% en alcohol); igual pero, Río Puerto Viejo, $10.440^{\circ} \mathrm{N}, 84.012^{\circ} \mathrm{W}$, el. $30 \mathrm{~m}, 10-11 . i i .1986$, Holzenthal, Morse, Fasth, 2 ơ, 1 \%; 19.vi.1986, Holzenthal, Heyn, Armitage, 2 ơ, 4 ᄋ; 10.ix.1986, M.M. Chavarría, 3 ơ, 1 \%; xi.1986, 1 O'; xii.1986, 3 ơ; P. N. Braulio Carrillo, Est. El Ceibo, Río Peje, $10.327^{\circ} \mathrm{N}$, $84.078^{\circ} \mathrm{W}$, el. 480 m, 29-31.v.1990, Holzenthal, Blahnik, Muñoz, 22 ơ, 33 \% (2 O’, 6 en alcohol); Est. Magsasay, Río Peje, $10.402^{\circ} \mathrm{N}$, $84.050^{\circ} \mathrm{W}$, el. $130 \mathrm{~m}, 25-26$.viii.1990, Holzenthal, Blahnik, Huisman, 14 ơ, 89 \& (5 ơ, 79 \% en alcohol); Limón: Río Telire and small trib., SE Suretka, $9.554^{\circ} \mathrm{N}, 82.892^{\circ} \mathrm{W}$, el. $48 \mathrm{~m}$, 1.ii.1986, Holzenthal, Morse, Fasth, $10^{\circ}, 1$ \%; Río Uatsi, ca. $8 \mathrm{~km}$ (air) W Bribri, $9.62^{\circ} \mathrm{N}$, $82.90^{\circ} \mathrm{W}$, el. $60 \mathrm{~m}$, 25.iii.1987, Holzenthal,Hamilton, Heyn, $20^{\circ}, 7$ ( $10^{\circ}, 2$ en alcohol); P. N. Braulio Carrillo, Queb. González, $10.160^{\circ} \mathrm{N}, 83.939^{\circ} \mathrm{W}$, el. $480 \mathrm{~m}, 12-14 . v .1990$, Holzenthal, Blahnik, 17 ơ, 31 \& (5 ơ, 16 en alcohol); Puntarenas: Queb. Pita, ca. 3 km 
(air) W Golfito, $8.642^{\circ} \mathrm{N}, 83.193^{\circ} \mathrm{W}$, el. $15 \mathrm{~m}$, 15.ii.1986, Holzenthal, Morse, Fasth, $90^{\prime \prime}, 14$ ㅇ ( $30^{7}, 6$ ㅇ e n alcohol); Río Negro, ca. $10 \mathrm{~km}$ (air) SSW Las Alturas, $8.950^{\circ} \mathrm{N}, 82.851^{\circ} \mathrm{W}$, el. 1035 m, 17.ii.1986, Holzenthal, Morse, Fasth, 1 o'; Río Ceibo, route 2, ca. 6 km W rd to Buenos Aires, $9.149^{\circ} \mathrm{N}, 83.377^{\circ} \mathrm{W}$, el. 250 m, 20.ii.1986, Holzenthal, Morse, Fasth, $10^{\circ}$, 27 \% (en alcohol); Queb. Tusa, route 2, ca. 3.5 km S Brujo, $9.064^{\circ} \mathrm{N}, 83.274^{\circ} \mathrm{W}$, el. $180 \mathrm{~m}$, 21.ii.1986, Holzenthal, Morse, Fasth, $10^{7}, 3$ ○ (en alcohol); Río Singrí, ca. $2 \mathrm{~km}$ (air) $\mathrm{S}$ Finca Helechales, $9.057^{\circ} \mathrm{N}, 83.082^{\circ} \mathrm{W}$, el. 720 m, 21.ii.1986, Holzenthal, Morse, Fasth, 2 o’; Río Guineal, ca $1 \mathrm{~km}$ (air) E Finca Helechales, $9.076^{\circ} \mathrm{N}, \quad 83.092^{\circ} \mathrm{W}$, el. $840 \mathrm{~m}$, 4.viii.1987, Holzenthal, Morse, Clausen, $10^{\circ}$, 3 \&; Río Jaba, at rock quarry 1.4 km (air) W Las Cruces, $8.79^{\circ} \mathrm{N}, 82.97^{\circ} \mathrm{W}$, el. $1150 \mathrm{~m}$, 14.vi.1986, Holzenthal, Heyn, Armitage, 3 o’, 4 \&; igual pero, 9.viii.1990, Holzenthal, Blahnik, Muñoz, 5 ơ', 4 \&; 15.iii.1991, Holzenthal, Muñoz, Huisman, 3 ơ, 6 \&; Río Bellavista, ca. $1.5 \mathrm{~km} \mathrm{NW}$ Las Alturas, $8.951^{\circ} \mathrm{N}$, $82.846^{\circ} \mathrm{W}$, el. $1400 \mathrm{~m}, 8-9 . i v .1987$, Holzenthal, Hamilton, Heyn, $10^{\circ}$; igual pero, 1617.iii.1991, Holzenthal, Muñoz, Huisman, 2 ơ, 3 \&; trib. to Río Bellavista in Las Alturas (road to quarry), $8.952^{\circ} \mathrm{N}, 82.848^{\circ} \mathrm{W}$, el. 1480 m, 13-14.viii.1990, Holzenthal, Blahnik, Muñoz, $10^{7}$ (en alcohol); Río Cotón, in Las Alturas, $8.938^{\circ} \mathrm{N}, 82.826^{\circ} \mathrm{W}$, el. $1360 \mathrm{~m}$, 12.viii.1990, Holzenthal, Blahnik, Muñoz, 1 O’ (en alcohol); Res. Biol. Carara, Queb. Bonita, $9.775^{\circ} \mathrm{N}, 84.605^{\circ} \mathrm{W}$, el. $35 \mathrm{~m}, 18$ 20.v.1990, Holzenthal, Blahnik, 6 o’, 21 ㅇ (2 ơ, 12 o en alcohol); igual pero, 11.iii.1991, Holzenthal, Muñoz, Huisman, 2 ơ, 4 \&; Río Carara, $4.3 \mathrm{~km}$ (rd) E Costanera Sur, $9.810^{\circ} \mathrm{N}$, $84.572^{\circ} \mathrm{W}$, el. $20 \mathrm{~m}, 12$. iii.1991, Holzenthal, Muñoz, Huisman, 1 ơ; San José: P. N. Braulio Carrillo, Est. Carrillo, Queb. El Molinete, $10.16^{\circ} \mathrm{N}, 83.96^{\circ} \mathrm{W}$, el. $700 \mathrm{~m}, 22-28 . v i i i .1986$, I. \& A. Chacón, 11 ơ, 7 \&; Res. Biol. Carara, Río Carara in Carara, $9.778^{\circ} \mathrm{N}, 84.531^{\circ} \mathrm{W}$, el. 200 m, 14.iii.1991, Holzenthal, Muñoz, Huisman, 3 ơ, 6 ㅇ.

\section{Leptonema cheesmanae Mosely}

Fig. 24; Mapa 1.

Leptonema cheesmanae Mosely 1933: 51, 52, figs. 148-153, ơ, Colombia: Isla Gorgona. (BMNH); Fischer 1963: 167; McElravy et al. 1981: 153; 1982: 307; Flint, McAlpine y Ross 1987: 72, figs. 711-717; Muñoz-Q. 1997: 130, fig. 6.

Diagnosis.-Adultos: Pequeños y con las alas translúcidas de color pardo claro a verde claro. Ala anterior de 14 a $18 \mathrm{~mm}$ de longitud, con un parche pequeño y redondeado de pelillos de color pardo oscuro sobre el área que rodea el ángulo anterior de la celda media $(\mathrm{cm})$, y el tercio apical cubierto levemente con pelillos pardo oscuros.

Phallus (Fig. 24): Esta especie y L. clorito Muñoz-Q. se caracterizan por la presencia de los procesos " $b-1$ ", " $b-2$ ", " $e-1$ " y " $e-2$ ", sin embargo difieren en la forma del proceso " $d$ ", como se explica en la diagnosis de L. clorito. Estas dos especies se distinguen de L. harpagum Flint, McAlpine y Ross, por la forma del proceso " $a$ ". Proceso " $a$ ", en L. cheesmanae y L. clorito, unilobulado, alargado, delgado, truncado y digitiforme; en L. harpagum, bilobulado y prominente. Proceso “ $j$ ” ausente en $L$. cheesmanae.

Distribución: Costa RICA (Alajuela, Guanacaste, Limón, San José); PANAMÁ; Colombia.

Material examinado: Costa RICA: Alajuela: Cerro Campana, ca. $6 \mathrm{~km}$ (air) NW Dos Ríos, $10.9^{\circ} \mathrm{N}, \quad 85.4^{\circ} \mathrm{W}$, el. $640 \mathrm{~m}, 15-$ 16.iii.1986, Holzenthal, Fasth, 1 ơ; Res. Forestal San Ramón, Río San Lorencito and tribs., $10.216^{\circ} \mathrm{N}, 84.607^{\circ} \mathrm{W}$, el. $980 \mathrm{~m}, 2-$ 4.vii.1986, Holzenthal, Heyn, Armitage, 5 o’; igual pero, 5-9.vii.1986, I. \& A. Chacón, 1 o'; 2-6.ix.1986, 9 ơ, 11 \&; 1-4.vii.1986, 2 ơ, 1 우; 24-27.ii.1987, 2 ơ; 30.iii.1987, Holzenthal, Hamilton, Heyn, 2 ơ (en alcohol) (INBio); 14.v.1990, Holzenthal, Blahnik, 28 ơ (10 en alcohol), 3 of; 28-30.vii.1990, Holzenthal, Blahnik, Muñoz, 3 ơ, 12 \&; 6-10.iii.1991, Holzenthal, Muñoz, Huisman, 4 ơ, 9 ᄋ; Guanacaste: P. N. Guanacaste, Est. Pitilla, Río Orosi, 

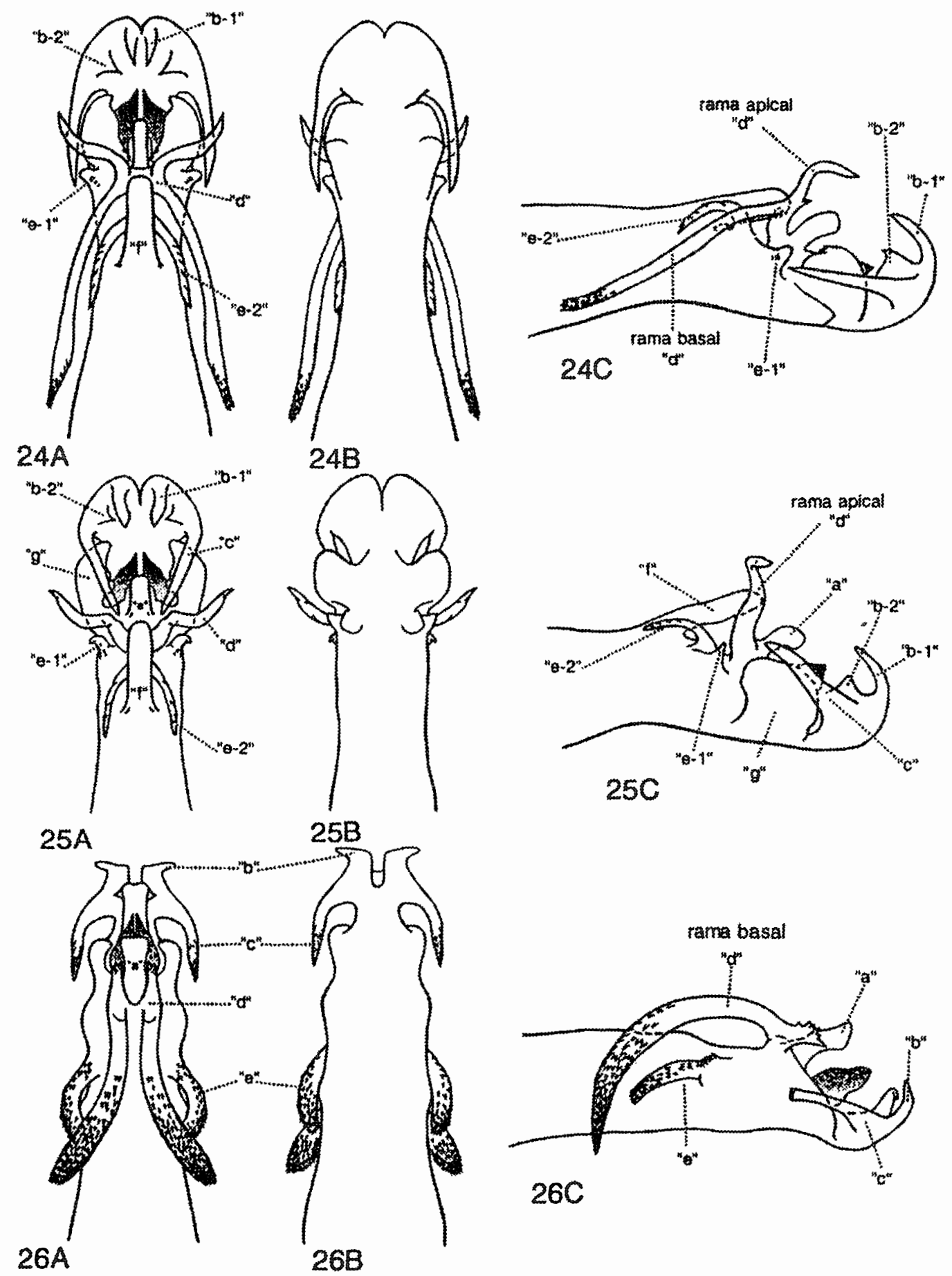

Figs. 24-26. Phallus: 24. L. cheesmanae, 25. L. clorito, 26. L. intermedium. -A., vista dorsal; B., vista ventral; C., vista lateral. 
$10.991^{\circ} \mathrm{N}, \quad 85.428^{\circ} \mathrm{W}, \quad$ el. $700 \mathrm{~m}, \quad 22-$ 25.v.1990, Holzenthal, Blahnik, 1 ơ; Est. Maritza, Río Tempisquito, $10.958^{\circ} \mathrm{N}, 85.497^{\circ} \mathrm{W}$, el. 550 m, 16.ii.1994, Muñoz, $40^{\prime \prime}, 3$ \& (en alcohol) (INBio); Limón: Res. Biol. Hitoy Cerere, Est. Miramar, $9.671^{\circ} \mathrm{N}, 83.030^{\circ} \mathrm{W}$, el.

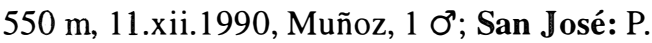
N. Braulio Carrillo, Est. Carrillo, Queb. Sanguijuela, $10.160^{\circ} \mathrm{N}, 83.963^{\circ} \mathrm{W}$, el. $800 \mathrm{~m}, 22-$ 28.viii.1986, I. \& A. Chacón, 3 ơ, 1 \&; igual pero, 27.iii.1987, Holzenthal, Hamilton, Heyn, 4 ơ.

\section{Leptonema clorito Muñoz-Q.} Fig. 25; Mapa 1.

Leptonema clorito Muñoz-Q. 1997: 117119, fig. 1, O’, Costa Rica: Alajuela (NMNH).

Diagnosis.-Adultos: Pequeños y con las alas translúcidas de color verde claro. Ala anterior de $17 \mathrm{~mm}$ de longitud, con un parche pequeño y redondeado de pelillos de color pardo oscuro sobre el área que rodea el ángulo anterior de la celda medial $(\mathrm{cm})$, y el tercio apical levemente con pelillos pardo oscuros.

Phallus (Fig. 25): Esta especie es muy similar a L. cheesmanae Mosely (Fig. 24), en ambas especies están presentes los procesos " $b-1$ ", " $b-2$ ", "e-l" y "e-2", sin embargo difieren en la forma del proceso " $d$ ". Proceso " $d$ ", en $L$. clorito, uniramificado, en vista lateral, consiste de una base delgada y dirigida dorsalmente, de donde emerge solamente la rama apical; la rama apical, en vista dorsal, bifurcada basalmente, las extensiones delgadas, sinuosas y dirigidas posterolateralmente. En $L$. cheesmanae, proceso " $d$ " biramificado, en vista lateral, consiste de una base delgada, curvada ligeramente y dirigida dorsalmente, de donde emergen las ramas apical y basal; la rama apical, en vista lateral, curvada y delgada, en vista dorsal, bifurcada basalmente, las extensiones sinuosas y dirigidas posterolateralmente; la rama basal, en vista dorsal, bifurcada basalmente, en vista lateral, delgada y dirigida anteroventralmente, más larga que la rama apical y sobrepasa la base del proceso " $f$ '. Proceso " $j$ " ausente en L. clorito.
Distribución: Costa Rica (Alajuela).

Material examinado: CosTA RICA: Alajuela: Cerro Campana, ca. $6 \mathrm{~km}$ (air) NW Dos Ríos, $10.9^{\circ} \mathrm{N}, 85.4^{\circ} \mathrm{W}$, el. $640 \mathrm{~m}, 15-$ 16.iii.1986, Holzenthal, Fasth, o', holotipo (NMNH).

\section{Leptonema complexum Mosely \\ Figs. 1 A, B, 27; Mapa 4.}

Leptonema complexum Mosely 1933: 5455, figs. 160-165, ơ, Panamá: Bugaba (BMNH); Fischer 1963: 168; Flint, McAlpine y Ross 1987: 72-73, figs. 718-724; Holzenthal 1988: 66.

Diagnosis.-Adultos (Figs. 1 A, B): Pequeños y con las alas translúcidas de color pardo claro a verde claro, principalmente basalmente. Ala anterior de 14 a $18 \mathrm{~mm}$ de longitud, con un parche pequeño, tenue y redondeado de pelillos pardos sobre el ángulo anterior de la celda medial $(\mathrm{cm})$.

Phallus (Fig. 27): Esta especie del Grupo complexum está ampliamente distribuida por el país y muy similar a $L$. forficulum Mosely (Fig. 28), pero difiere en la forma del proceso " $e$ ". Proceso " $e$ ", en L. complexum, con uno o más procesos basodorsales, éstos de muy pequeños a conspicuos, y en forma de espinas; y en $L$. forficulum, sin procesos basodorsales. Según Flint et al. (1987), la forma de la rama apical del proceso " $d$ ", difiere en estas dos especies. Especímenes de L. complexum examinados para esta revisión presentaron variación en la forma de la rama apical del proceso " $d$ ", esta variación se solapa con la descripción dada por Flint et al. para la rama apical de L. forficulum, lo que no nos permitió utilizarla como un carácter diagnóstico. Por las recolectas revisadas, se observó que $L$. complexum es mucho más abundante que $L$. forficulum. Estas dos especies se distinguen de L. furciligerum Flint, McAlpine y Ross, por la forma y origen del proceso " $e$ ", como se indica en la diagnosis de esta última especie. En L. complexum, procesos " $f$ " y " $j$ " ausentes. 


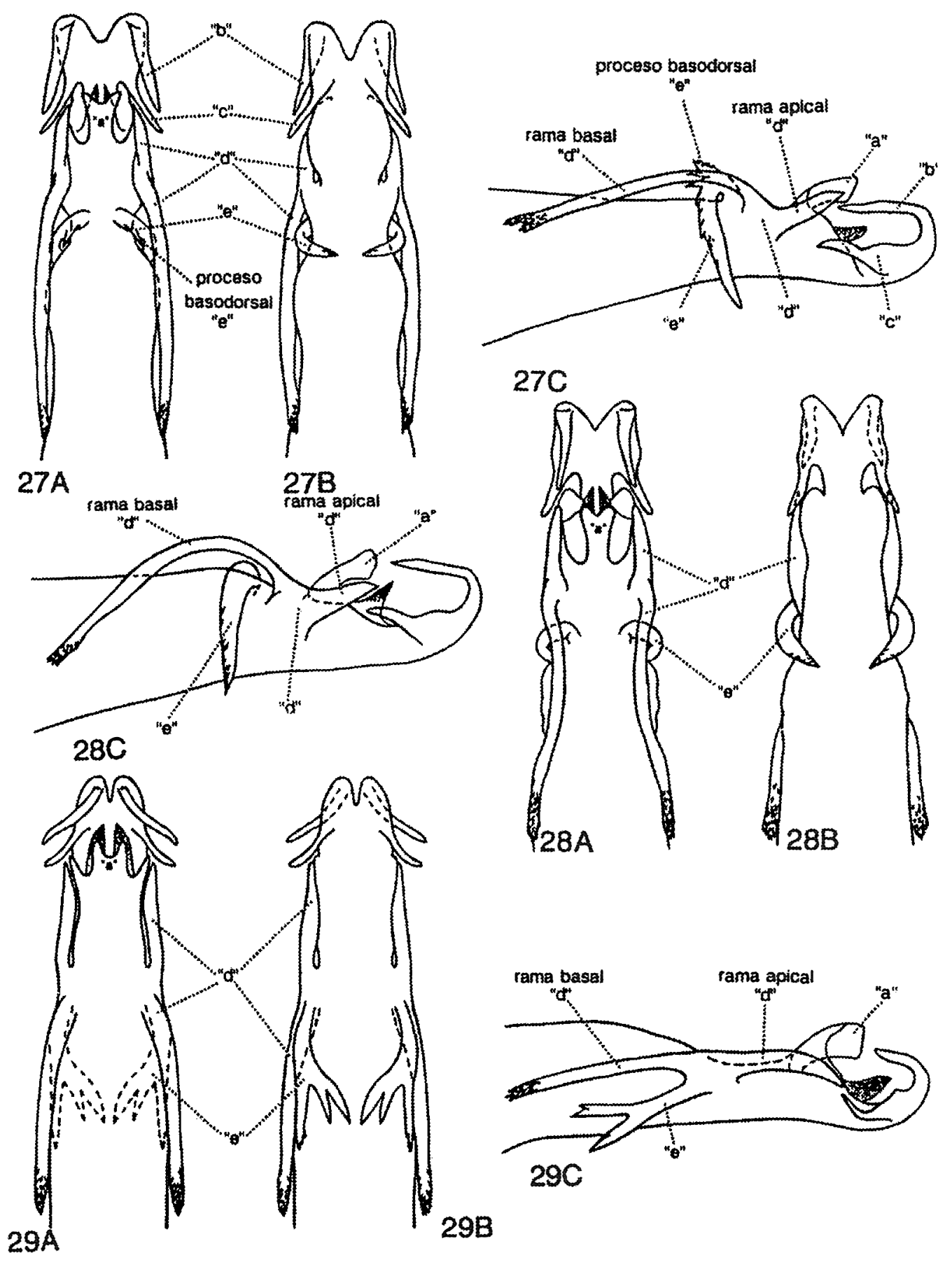

Figs. 27-29. Phallus: 27. L. complexum, 28. L. forficulum, 29. L. furciligerum. -A., vista dorsal; B., vista ventral; C., vista lateral. 


\section{COSTA RICA MAPA 1}

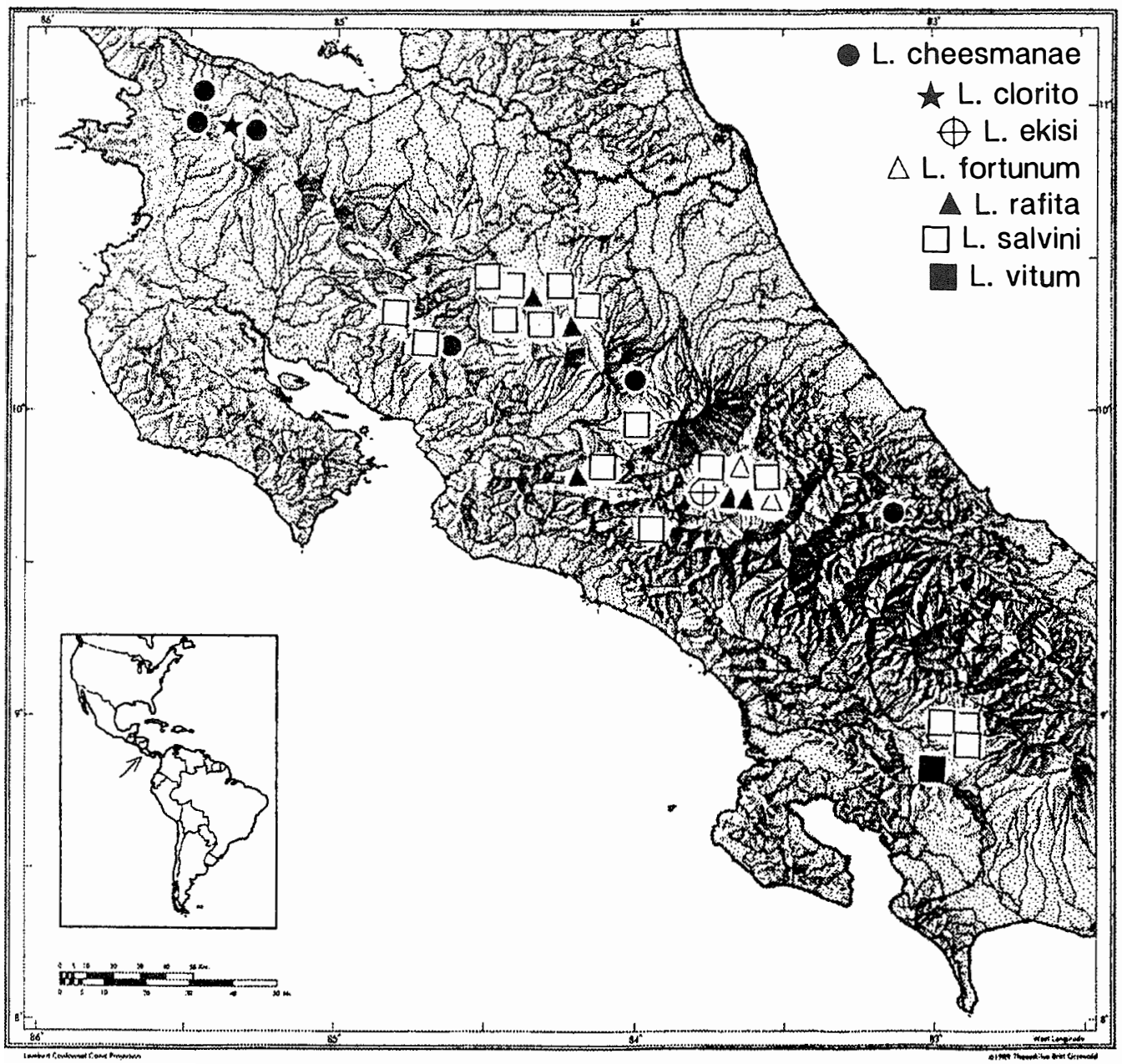

Fig. 30. Mapa 1, con la distribución conocida de los adultos de L. cheesmanae, L. clorito, L. ekisi, L. fortunum, L. rafita, L. salvini y $L$. vitum.

Distribución: Costa RICA (Alajuela, Cartago, Guanacaste, Heredia, Limón, Puntarenas, San José); Panamá.

Material examinado: Costa RICA: Alajuela: P. N. Rincón de la Vieja, Queb. Provisión, $10.769^{\circ} \mathrm{N}, \quad 85.281^{\circ} \mathrm{W}$, el. $810 \mathrm{~m}$, 4.iii.1986, Holzenthal, Fasth, $30^{\circ}, 12$ \& ( $20^{\circ}, 4$ o en alcohol); Cerro Campana, ca. 6 km (air) NW Dos Ríos, $10.9^{\circ} \mathrm{N}, 85.4^{\circ} \mathrm{W}$, el. $640 \mathrm{~m}, 15-$ 16.iii.1986, Holzenthal, Fasth, 2 ơ, 15 \&; Cerro Campana, Río Bochinche trib., ca. $6 \mathrm{~km}$ (air) NW Dos Ríos, $10.945^{\circ} \mathrm{N}, 85.413^{\circ} \mathrm{W}$, el.
600 m, 22-23.vii.1987, Holzenthal, Morse, Clausen, $310^{\circ}, 42$ ( $30^{\circ}, 7 \%$ en alcohol); Res. Forestal San Ramón, Río San Lorencito \& tribs., $10.216^{\circ} \mathrm{N}, 84.607^{\circ} \mathrm{W}$, el. $980 \mathrm{~m}, 5-$ 9.vii.1986, I. \& A. Chacón, 19 ơ, 18 \&; igual pero, 2-6.ix.1986, 40 ơ, 31 \&; 1-4.x.1986, 12 ơ, 21 \%; xii.1986, 1 ơ, 2 \%; 24-27.ii.1987, 4 ơ, 12 \&; 30.iii.-1.iv.1987, Holzenthal, Hamilton, Heyn, 70 ơ, 67 \& (12 ơ, 5 \& en alcohol); 14.v.1990, Holzenthal, Blahnik, 27 ơ, 41 \% (11 ơ, 3 \& en alcohol); 28-30.vii.1990, Holzenthal, Blahnik, Muñoz, 19 ơ, 42 \&; 6-10.iii.1991, 


\section{COSTA RICA MAPA 2}

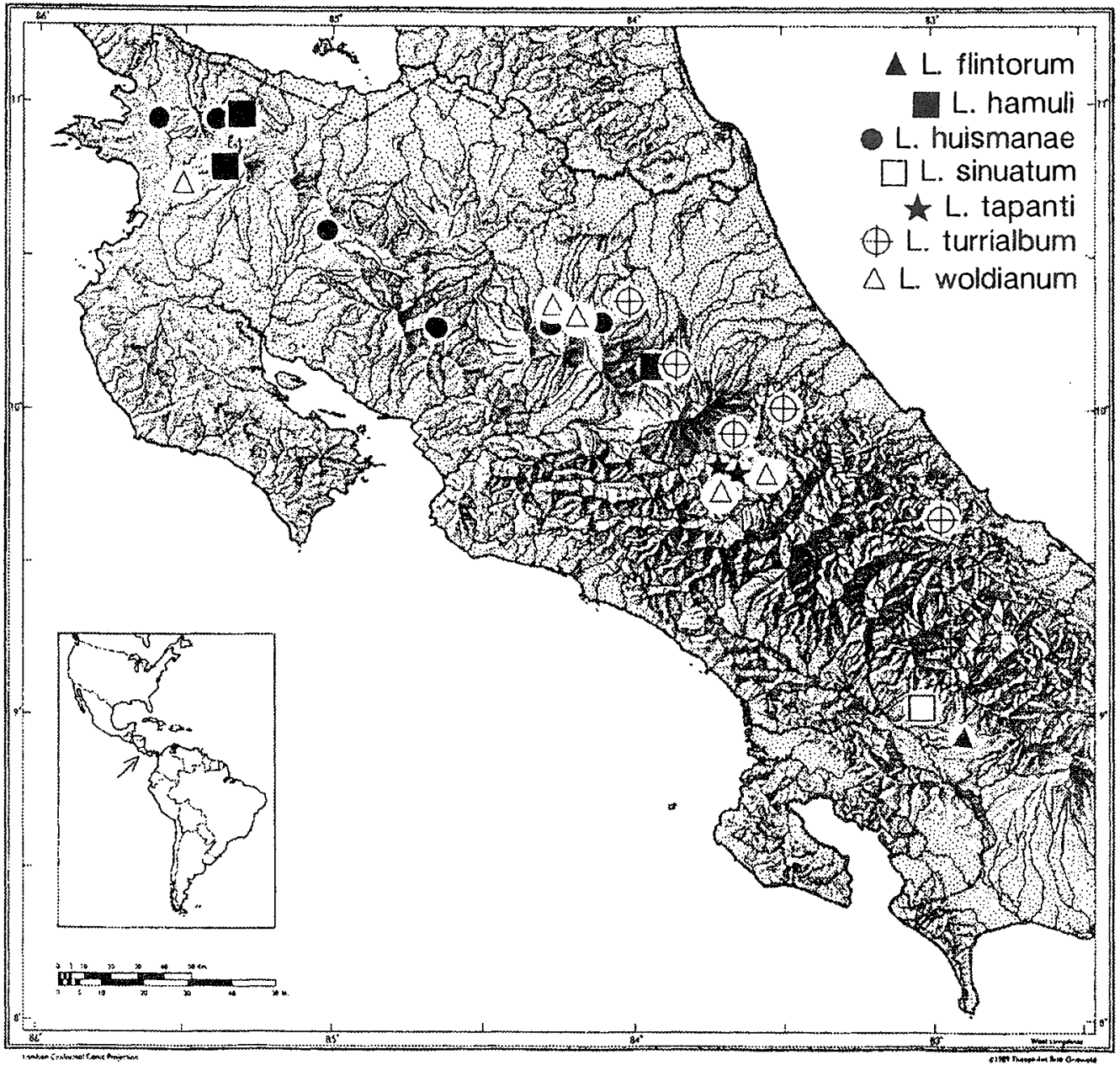

Fig. 31. Mapa 2, con la distribución conocida de los adultos de L. flintorum, L. hamuli, L. huismanae, $L$. sinuatum, L. tapan$t i, L$. turrialbum y $L$. woldianum.

Holzenthal, Muñoz, Huisman, 25 ơ, 56 ๆ (10 o’, 14 \& en alcohol); Cartago: Río Chitaría, rt 10, 10 km NW Río Reventazón, el. 740 m, $9.920^{\circ} \mathrm{N}, 83.604^{\circ} \mathrm{W}, 21.1 i i .1991$, Holzenthal, Muñoz, Huisman, 22 ơ, 17 \%; Queb. Platanillo, ca $5 \mathrm{~km}$ E Moravia de Chirripó, $9.821^{\circ} \mathrm{N}$, $83.407^{\circ} \mathrm{W}$, el. $1130 \mathrm{~m}$, 6.viii.1987, Holzenthal, Morse, Clausen, $60^{\prime \prime}, 9$ ( $20^{\prime \prime}, 7 \%$ en alcohol); Guanacaste: Queb. García, 10.6 km. ENE Quebrada Grande, $10.862^{\circ} \mathrm{N}, 85.428^{\circ} \mathrm{W}$, el. 470 m, 8.iii.1986, Holzenthal, Fasth, 18 ơ; Río Los Ahogados, $11.3 \mathrm{~km}$ ENE Quebrada Grande, $10.865^{\circ} \mathrm{N}, 85.423^{\circ} \mathrm{W}$, el. $470 \mathrm{~m}$, 7.iii.1986,
Holzenthal, Fasth, $60^{\circ}, 6$ ( $10^{\circ}, 3$ en alcohol); igual pero, 26.vi.1986, Holzenthal, Heyn, Armitage, 1 Ơ; P. N. Guanacaste, Est. Maritza, Río Tempisquito, $10.958^{\circ} \mathrm{N}, 85.497^{\circ} \mathrm{W}$, el. 550 m, 19-20.vii.1987, Holzenthal, Morse, Clausen, 15 ơ (2 en alcohol), 17 \%; igual pero, 3031.viii.1990, Huisman, Blahnik, Quesada, 19 ơ, 12 \& (15 ơ, 10 \& en alcohol); Est. Maritza, Volcán Orosí, 2.vi.1986, D.H. Janzen, 1 O'; Est. Pitilla, Río Orosí, $10.991^{\circ} \mathrm{N}, 85.428^{\circ} \mathrm{W}$, el. 700 m, 22-25.v.1990, Holzenthal, Blahnik, 33 ơ; Z. P. Tenorio, tribs. to Río San Lorenzo, 6 $\mathrm{km}$ NW Tierras Morenas, $10.61^{\circ} \mathrm{N}, 84.98^{\circ} \mathrm{W}$, 


\section{COSTARICA MAPA 3}

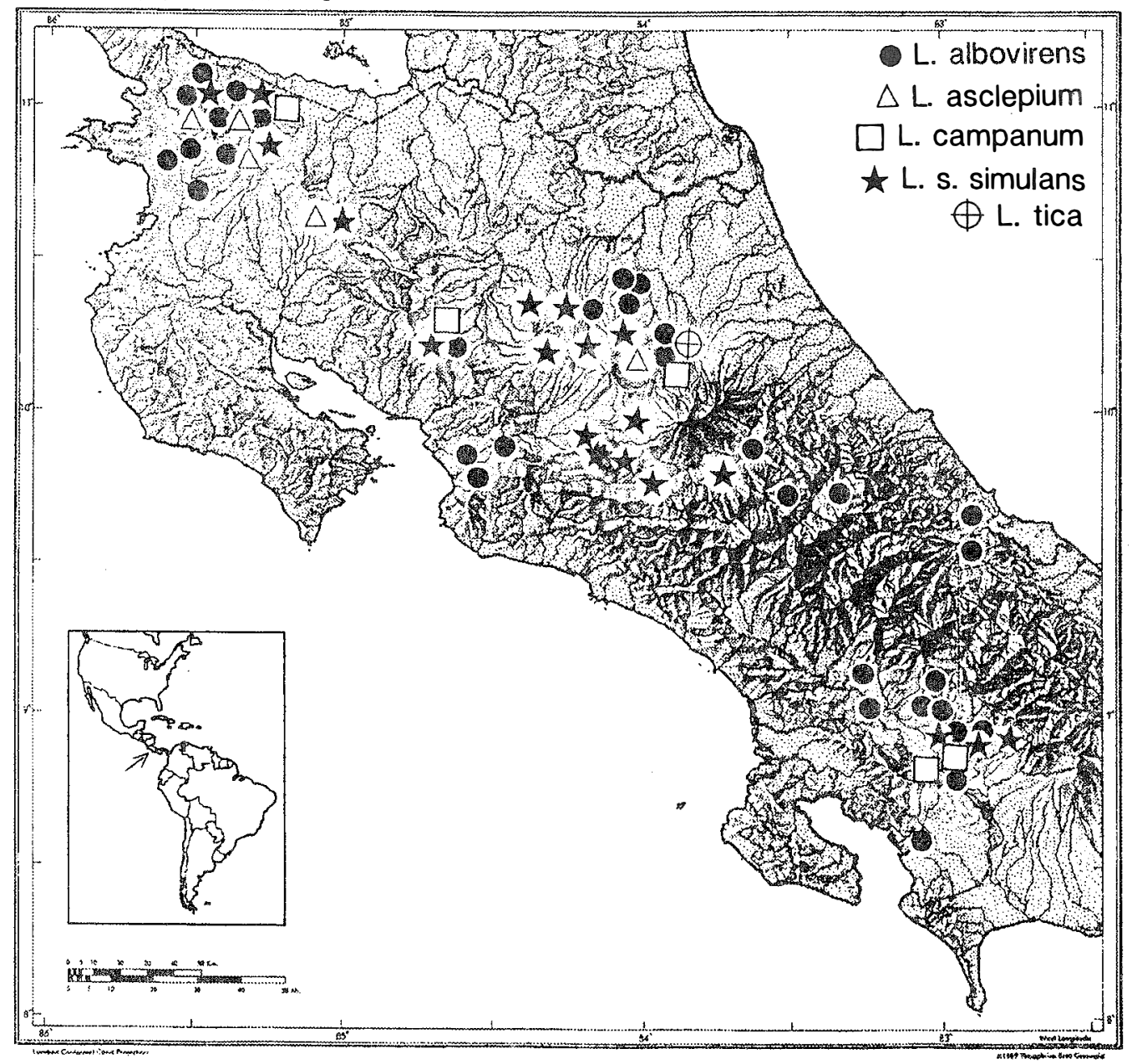

Fig. 32. Mapa 3, con la distribución conocida de los adultos de L. albovirens, L. asclepium, L. campanum, L. s. simulans y L. tics.

el. 900 m, 17-19.ii.1992, Holzenthal, Muñoz, Kjer, 1 O', 1 \&; Heredia: P. N. Braulio Carrillo, Est. Magsasay, Río Peje, $10.402^{\circ} \mathrm{N}, 84.050^{\circ} \mathrm{W}$, el. $130 \mathrm{~m}, 25-26 . v i i i .1990$, Holzenthal, Blah-

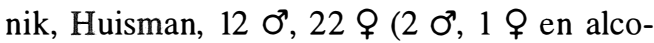
hol); Limón: Río Banano, 16 km WSW Bomba, $9.888^{\circ} \mathrm{N}, 83.167^{\circ} \mathrm{W}$, el. $150 \mathrm{~m}$, 26.iii. 1987 , Holzenthal, Hamilton, Heyn, 2 O'; P. N. Braulio Carrillo, Queb. González, $10.160^{\circ} \mathrm{N}$, $83.939^{\circ} \mathrm{W}$, el. $480 \mathrm{~m}, 12-14 . v .1990$, Holzenthal, Blahnik, 19 ơ, 16 \& (1 0", 8 \& en alcohol); Res. Biol. Barbilla, Río Dantas, 15 km (rd) S Pacuarito, $9.994^{\circ} \mathrm{N}, 83.443^{\circ} \mathrm{W}$, el. $300 \mathrm{~m}, 27$ - 30.i.1992, Holzenthal, Muñoz, Kjer, 2 ơ, 2 ᄋ; Puntarenas: Río Guineal, ca. $1 \mathrm{~km}$ (air) E Finca Helechales, $9.076^{\circ} \mathrm{N}, 83.092^{\circ} \mathrm{W}$, el. $840 \mathrm{~m}$, 22.ii.1986, Holzenthal, Morse, Fasth, 1 ơ, 2 ᄋ (en alcohol); igual pero, 4.viii.1987, Holzenthal, Morse, Clausen, 2 ơ; Río Jaba, at rock quarry $1.4 \mathrm{~km}$ (air) W Las Cruces, $8.79^{\circ} \mathrm{N}$, $82.97^{\circ} \mathrm{W}$, el. $1150 \mathrm{~m}, 15$. iii.1991, Holzenthal, Muñoz, Huisman, $10^{\circ}, 1$ \%; Res. Biol. Carara, Queb. Bonita, $9.775^{\circ} \mathrm{N}, 84.605^{\circ} \mathrm{W}$, el. $35 \mathrm{~m}$, 18-20.v.1990, Holzenthal, Blahnik, 2 O’; igual pero, 11.iii.1991, Holzenthal, Muñoz, Huisman, 1 O’; Río Cotón, in Las Alturas, $8.938^{\circ} \mathrm{N}$, 


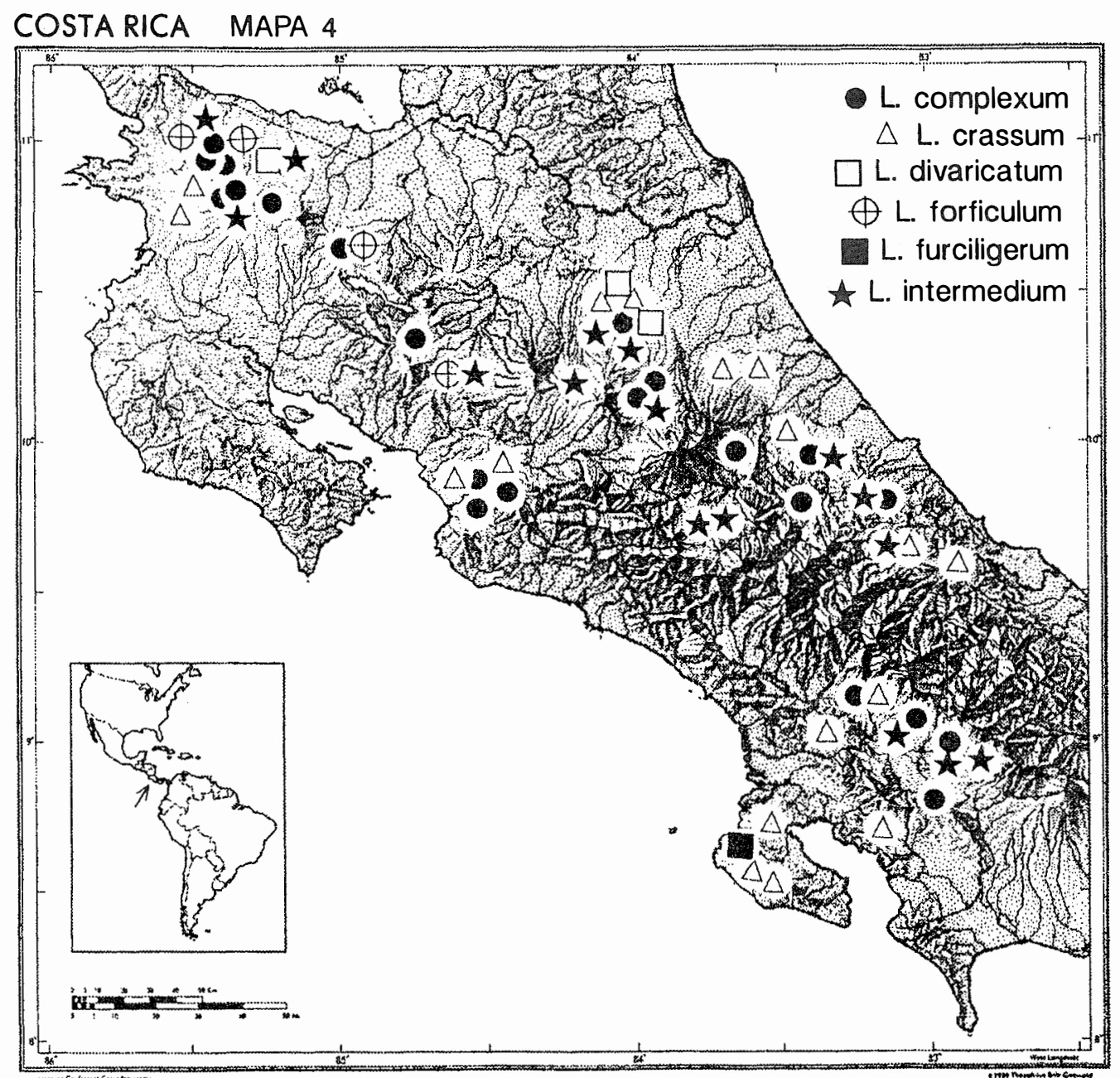

Fig. 33. Mapa 4, con la distribución conocida de los adultos de L. complexum, L. crassum, L. divaricatum, $L$. fortificulum, L. furciligerum y L. intermedium.

$82.826^{\circ} \mathrm{W}$, el. $1360 \mathrm{~m}, 16$. ii.1986, Holzenthal, Morse, Fasth, 9 ơ, 4 우 (en alcohol); Río Platanar, Salitre, $6.5 \mathrm{~km} \mathrm{NE}$ Buenos Aires, $9.115^{\circ} \mathrm{N}$, 83. $281^{\circ} \mathrm{W}$, el. 450 m, 8-9.vi.1992, Muñoz, 3 o?; San José: P. N. Braulio Carrillo, Queb. El Molinete, $10.16^{\circ} \mathrm{N}, 83.96^{\circ} \mathrm{W}$, el. $700 \mathrm{~m}, 22-$ 28.viii.1986, I. \& A. Chacón, $10^{7}$; Queb. Sanguijuela, Est. Carrillo, $10.160^{\circ} \mathrm{N}, 83.963^{\circ} \mathrm{W}$, el. 800 m, 27.iii.1987, Holzenthal, Hamilton, Heyn, 3 ơ, 2 \&; Res. Biol. Carara, Río del Sur, $1.5 \mathrm{~km}$ (rd.) $\mathrm{S}$ of Carara, $9.769^{\circ} \mathrm{N}, 84.531^{\circ} \mathrm{W}$, el. $160 \mathrm{~m}$, 13.iii.1991, Holzenthal, Muñoz, Huisman, 2 ơ (1 en alcohol), 2 \&; Río Carara in Carara, $9.778^{\circ} \mathrm{N}, 84.531^{\circ} \mathrm{W}$, el. $200 \mathrm{~m}$, 14.iii.1991, Holzenthal, Muñoz, Huisman, 2 ơ, 1 ㅇ (en alcohol).

\section{Leptonema forficulum Mosely}

Fig. 28; Mapa 4.

Leptonema forficulum Mosely 1933: 5254, figs. 154-159 (como L. forficula), ơ, Panamá: Cabima (MCZ); Fischer 1963: 168; Flint, McAlpine y Ross 1987: 73, figs. 725-730; Holzenthal 1988: 67. 
Diagnosis.-Adultos: Pequeños y con las alas translúcidas de color pardo claro a verde claro, principalmente basalmente. Ala anterior de 14 a $18 \mathrm{~mm}$ de longitud, con un parche pequeño, tenue y redondeado de pelillos pardos sobre el ángulo anterior de la celda medial $(\mathrm{cm})$.

Phallus (Fig. 28): Esta especie, L. complexum Mosely, y L. furciligerum Flint, McAlpine y Ross, son similares, sin embargo difieren en la forma del proceso " $e$ ", como se especifica en las diagnosis de esas especies. En $L$. forficulum, procesos " $f$ " y " $j$ " ausentes.

Distribución: CosTA Rica (Alajuela, Guanacaste); PANAMÁ.

Material examinado: CostA RICA: Alajuela: Cerro Campana, ca. $6 \mathrm{~km}$ (air) NW Dos Ríos, $10.9^{\circ} \mathrm{N}, 85.4^{\circ} \mathrm{W}$, el. $640 \mathrm{~m}, 15-$ 16.iii.1986, Holzenthal, Fasth, $10^{\text {’3 }}$ Res. Forestal San Ramón, Río San Lorencito \& tribs., $10.216^{\circ} \mathrm{N}, 84.607^{\circ} \mathrm{W}$, el. $980 \mathrm{~m}, 2-6 . i x .1986$, I. \& A. Chacón, 1 ơ; igual pero, 30.iii:1.iv.1987, Holzenthal, Hamilton, Heyn, 2 o"; Guanacaste: P. N. Guanacaste, Est. Maritza, Río Tempisquito, $10.958^{\circ} \mathrm{N}, 85.497^{\circ} \mathrm{W}$, el. 550 m, 13-16.vii.1992, Muñoz, 1 o'; igual pero, 19-20.vii.1987, Holzenthal, Morse, Clausen, 2 ơ; Zona Protectora Tenorio, tribs. to Río San Lorenzo, $6 \mathrm{~km}$ NW Tierras Morenas, $10.61^{\circ} \mathrm{N}$, $84.98^{\circ} \mathrm{W}$, el. $900 \mathrm{~m}, 17-19$. ii.1992, Holzenthal, Muñoz, Kjer, 10 \%".

\section{Leptonema furciligerum Flint, McAlpine y Ross}

Fig. 29; Mapa 4.

Leptonema furciligerum Flint, McAlpine y Ross 1987: 73-74, figs. 731-738, ơ, Costa Rica: Puntarenas (NMNH); Holzenthal 1988: 67.

Diagmosis.-Adultos: Pequeños y con las alas translúcidas de color pardo claro y verde claro basalmente. Ala anterior de 12 a $16 \mathrm{~mm}$ de longitud, con un parche pequeño y redondeado de pelillos pardo oscuros sobre el "nygma" (entre las venas $R_{4}-R_{5}$ ), y otro parche pequeño y redondeado de pelillos pardo oscuros sobre el ángulo anterior de la celda medial (" $\mathrm{cm}$ ").
Phallus (Fig. 29): Esta especie, L. complexum Mosely'(Fig. 27) y L. forficulum Mosely (Fig. 28), aunque parecidas, se distinguen por la forma y origen del proceso " $e$ ". Proceso " $e$ ", en L. furciligerum, en vista lateral, emerge lateralmente, dirigido anteroventralmente y dividido apicalmente; en L. complexum y L. forficulum, emerge dorsolateralmente, dirigido ventralmente y no dividido apicalmente. Procesos " $f$ " y " $j$ " ausentes en L. furciligerum.

Distribución: Costa Rica (Puntarenas).

Material examinado: Costa Rica: Puntarenas: P. N. Corcovado, Piedra el Arco, $8.582^{\circ} \mathrm{N}, 83.709^{\circ} \mathrm{W}$, el. $20 \mathrm{~m}, 10-11 . i v .1989$, Holzenthal, Blahnik, 4 ơ, 1 \%.

\section{Leptonema intermedium Mosely}

Fig. 26; Mapa 4.

Leptonema intermedium Mosely 1933: 4849, figs. 137-141, ơ, Ecuador: Chimbo (ZSZMH); Fischer 1963: 169; 1972: 156; Wiedner 1964: 84; McElravy et al. 1981: 153; 1982: 307, 310, 312; Flint, McAlpine y Ross 1987: 74-75, figs. 754-760; Holzenthal 1988: 67.

Diagnosis.-Adultos: Pequeños y con las alas translúcidas de color pardo claro a verde claro. Ala anterior de 13 a $16 \mathrm{~mm}$ de longitud, con un parche pequeño y tenue de pelillos pardos oscuros sobre el ángulo anterior de la celda medial $(\mathrm{cm})$, y el tercio apical con pelillos pardos.

Phallus: (Fig. 26): Esta especie se diferencia de $L$. rosenbergi Mosely por la presencia del proceso " $f$ '. Proceso " $f$ ", en $L$. intermedium, ausente; y en $L$. rosenbergi, mesodorsal, corto y dirigido dorsalmente. La forma del proceso " $e$ " es similar en $L$. intermedium y $L$. rosenbergi, sin embargo, el proceso " $e$ " de $L$. rosenbergi no aparece dibujado en las figuras 134 y 135 de Mosely (1933), 763 y 764 de Flint et al. (1987). Procesos "c", “ $d$ ", “ $f$ ", “ $j$ ” ausentes en $L$. intermedium.

Distribución: CosTA Rica (Alajuela, Cartago, Guanacaste, Heredia, Limón, Puntarenas, San José); Panamá; Colombia; Ecuador. 
Material examinado: CosTA RicA: Alajuela: Río Pizote, ca. $5 \mathrm{~km}$ N Dos Ríos, $10.948^{\circ} \mathrm{N}, 85.291^{\circ} \mathrm{W}$, el. $470 \mathrm{~m}$, 9.iii.1986, Holzenthal, Fasth, $20^{7}$; Cerro Campana, ca. $6 \mathrm{~km}$ (air) NW Dos Ríos, $10.9^{\circ} \mathrm{N}, 85.4^{\circ} \mathrm{W}$, el. $640 \mathrm{~m}$, 15-16.iii.1986, Holzenthal, Fasth, 2 ơ; Cerro Campana, Río Bochinche trib., ca. $6 \mathrm{~km}$ (air) NW Dos Ríos, $10.945^{\circ} \mathrm{N}, 85.413^{\circ} \mathrm{W}$, el. $600 \mathrm{~m}, 22-$ 23.vii.1987, Holzenthal, Morse, Clausen, 2 ơ; Río Sarapiquí, ca. 2 km SE Cariblanco, $10.299^{\circ} \mathrm{N}, 84.172^{\circ} \mathrm{W}$, el. $710 \mathrm{~m}, 22 . i i i .1986$, Holzenthal, Fasth, 4 ơ; igual pero, 22.vi.1986, Holzenthal, Heyn, Armitage, 1 O’, 3 ㅇ (en alcohol); Res. Forestal San Ramón, Río San Lorencito \& tribs., $10.216^{\circ} \mathrm{N}$, $84.607^{\circ} \mathrm{W}$, el. $980 \mathrm{~m}, 2-4 . v i i .1986$, Holzenthal, Heyn, Armitage, 24 O’, 33 \%; Queb. Latas, $8.9 \mathrm{~km}$ NE Bajos del Toro, $10.269^{\circ} \mathrm{N}$, $84.260^{\circ} \mathrm{W}$, el. $1030 \mathrm{~m}, 6 . i x .1990$, Holzenthal, Blahnik, Huisman, 2 ơ, 1 \%; Cartago: Res. Tapantí, Queb. Segunda, @ administration building, $9.761^{\circ} \mathrm{N}, 83.787^{\circ} \mathrm{W}$, el. 1250 m, 9-10.v.1990, Holzenthal, Blahnik, 1 ơ; igual pero, Queb. Palmitos \& falls, $9.72^{\circ} \mathrm{N}, 83.78^{\circ} \mathrm{W}$, el. $1400 \mathrm{~m}, 1-2 . v i i i .1990$, Holzenthal, Blahnik, Muñoz, 1 0', 2 \%; Guanacaste: Río Los Ahogados, $11.3 \mathrm{~km}$ ENE Quebrada Grande, $10.865^{\circ} \mathrm{N}$, $85.423^{\circ} \mathrm{W}$, el. $470 \mathrm{~m}, 26 . v i .1986$, Holzenthal, Heyn, Armitage, 2 ơ; Queb. García, $10.6 \mathrm{~km}$ ENE Quebrada Grande, $10.862^{\circ} \mathrm{N}$, $85.428^{\circ} \mathrm{W}$, el. $470 \mathrm{~m}, 8$. iii.1986, Holzenthal, Fasth, 10 ơ (9 ơ, 3 \& en alcohol); P. N. Guanacaste, Est. Maritza, Río Tempisquito, $10.958^{\circ} \mathrm{N}, 85.497^{\circ} \mathrm{W}$, el. $550 \mathrm{~m}, 13-$ 16.vii.1992, Muñoz, 1 ơ; Est. Pitilla, Río Orosí, $10.991^{\circ} \mathrm{N}, 85.428^{\circ} \mathrm{W}$, el. $700 \mathrm{~m}, 22-$ 25.v.1990, Holzenthal, Blahnik, 2 O'; Heredia: Río Bijagual, on road to Magsasay, $10.408^{\circ} \mathrm{N}, 84.076^{\circ} \mathrm{W}$, el. $140 \mathrm{~m}, 12.1 i .1986$, Holzenthal, Morse, Fasth, $10^{\circ}$ (en alcohol); Río Sarapiquí, $7 \mathrm{~km} \mathrm{~W}$ Pto Viejo, $10.452^{\circ} \mathrm{N}, 84.067^{\circ} \mathrm{W}$, el. $50 \mathrm{~m}, 11 . i i .1986$, Holzenthal, Morse, Fasth, $10^{\circ}, 1$ \& (en alcohol); Est. Biol. La Selva, Queb. Sura, $10.437^{\circ} \mathrm{N}, \quad 84.010^{\circ} \mathrm{W}$, el. $50 \mathrm{~m}, \quad 20-$ 21.vi.1986, Holzenthal, Heyn, Armitage, 3 ơ (2 O', 5 \% en alcohol); P. N. Braulio Carrillo, Est. El Ceibo, Río Peje, $10.327^{\circ} \mathrm{N}$, $84.078^{\circ} \mathrm{W}$, el. $480 \mathrm{~m}, 29-31 . v .1990$, Holzenthal, Blahnik, Muñoz, 6 o", 13 \%; Est. Magsasay, Río Peje, $10.402^{\circ} \mathrm{N}, 84.050^{\circ} \mathrm{W}$, el. $130 \mathrm{~m}, 25-26$.viii.1990, Holzenthal, Blahnik, Huisman, 15 ơ, 10 \& (en alcohol); Limón: Res. Biol. Hitoy Cerere, Río Cerere, $9.671^{\circ} \mathrm{N}, 83.028^{\circ} \mathrm{W}$, el. $90 \mathrm{~m}$, xii. 1990 , Muñoz, $10^{\prime}, 8$ \& (en alcohol); P. N. Braulio Carrillo, Queb. González, $10.160^{\circ} \mathrm{N}$, $83.939^{\circ} \mathrm{W}$, el. $480 \mathrm{~m}, 12-14 . v .1990$, Holzenthal, Blahnik, 6 O’ (3 en alcohol), 7 \%; Res. Biol. Barbilla, Río Dantas, 15 km (rd) S Pacuarito, $9.994^{\circ} \mathrm{N}, 83.443^{\circ} \mathrm{W}$, el. $300 \mathrm{~m}$, 27-30.i.1992, Holzenthal, Muñoz, Kjer, 3 ơ, 1 \&; Río Banano, 16 km WSW Bomba, $9.888^{\circ} \mathrm{N}, 83.167^{\circ} \mathrm{W}$, el. $150 \mathrm{~m}, 26 . i i i .1987$, Holzenthal, Hamilton, Heyn, 1 ơ; Puntarenas: Río Negro, ca. $10 \mathrm{~km}$ (air) SSW Las Alturas, $8.950^{\circ} \mathrm{N}, 82.851^{\circ} \mathrm{W}$, el. $1035 \mathrm{~m}$, 17.ii.1986, Holzenthal, Morse, Fasth, 100 , 10 \%; Río Singrí, ca. $2 \mathrm{~km}$ (air) S Finca Helechales, $9.057^{\circ} \mathrm{N}, 83.082^{\circ} \mathrm{W}$, el. $720 \mathrm{~m}$, 21.ii.1986, Holzenthal, Morse, Fasth, $190^{\circ}$, 29 \% (9 0̛, 25 \% en alcohol); Río Guineal, ca. $1 \mathrm{~km}$ (air) E Finca Helechales, $9.076^{\circ} \mathrm{N}$, $83.092^{\circ} \mathrm{W}$, el. $840 \mathrm{~m}, 22$. ii.1986, Holzenthal, Morse, Fasth, 4 ơ (1 en alcohol), 4 \%; igual pero, 4.viii.1987, Holzenthal, Morse, Clausen, 19 ơ, 5 \%; Río Cotón, in Las Alturas, $8.938^{\circ} \mathrm{N}, 82.826^{\circ} \mathrm{W}$, el. $1360 \mathrm{~m}$, 16.ii.1986, Holzenthal, Morse, Fasth, 38 o", 42 \& (3 ơ, 7 \% en alcohol); igual pero, 12.viii.1990, Holzenthal, Blahnik, Muñoz, 6 ơ, 8 \& (1 ơ, 5 ᄋ en alcohol); 18.iii.1991, Holzenthal, Muñoz, Huisman, 1 ơ, 9 \&; Río Bellavista, ca. $1.5 \mathrm{~km} \mathrm{NW}$ Las Alturas, $8.951^{\circ} \mathrm{N}, \quad 82.846^{\circ} \mathrm{W}$, el. $1400 \mathrm{~m}, \quad 8-$ 9.iv.1987, Holzenthal, Hamilton, Heyn, 53 ơ, 41 \& (10 ơ, 20 \% en alcohol); trib. to Río Bellavista in Las Alturas (road to quarry), $8.952^{\circ} \mathrm{N}, 82.848^{\circ} \mathrm{W}$, el. $1480 \mathrm{~m}, 19$. iii. 1991 , Holzenthal, Muñoz, Huisman, 4 ơ, 7 9. 
Clave para la identificación de los machos de las especies conocidas de Leptonema Guérin, en Costa Rica (basada en el phallus)

1. -Phallus tubular, sin procesos " $a$ ", " $b$ ", "c", " $d$ ", " $e$ ", " $f$ ", " $g$ ", " $j "$

2

- Phallus tubular, con uno o más procesos "a", "b", "c", "d", "e", "f", "g", "j" ........................................................... 3

2. -Proceso dorsal del conducto eyaculatorio en vista lateral, subtriangular, corto, no alcanza el margen dorsal del phallus; proceso ventral del conducto eyaculatorio en vista lateral, sinuoso, ápice apenas alcanza el margen apical del phallus (Fig. 5, Mapa 4)

L. crassum

- Proceso dorsal del conducto eyaculatorio en vista lateral, curvado ligeramente, alargado, alcanza el margen dorsal del phallus; proceso ventral del conducto eyaculatorio en vista lateral, curvado basalmente, erguido, digitiforme, largo, sobrepasa el margen dorsoapical del phallus (Fig. 6, Mapa 4)

L. divaricatum

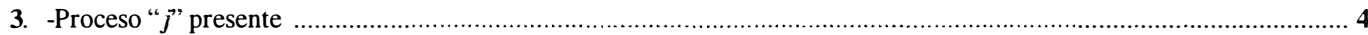

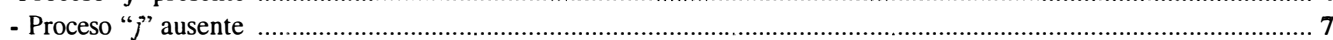

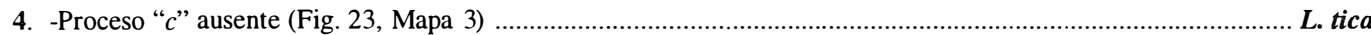

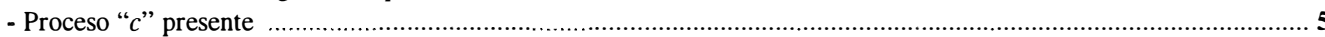

5. -Proceso " $b$ " corto, dirigido posterodorsalmente (Fig. 21, Mapa 3) …………………............................... L. campanum - Proceso " $b$ " largo, dirigido anteroventralmente, alcanza el margen ventral del phallus .......................................... 6

6. -Proceso " $b$ " en vista lateral, arqueado; proceso " $g$ " en vista lateral, con el ápice puntiagudo; proceso " $j$ " en vista dorsal, con una o más proyecciones laterales, prof undamente bifurcado apicalmente (más de 1/3 del largo del proceso) (Fig. 20, Mapa 3)

L. asclepium

- Proceso " $b$ " en vista lateral, curvado fuertemente; proceso " $g$ " en vista lateral, con el ápice truncado; proceso " $j$ " en vista dorsal, sin proyecciones laterales, levemente bifurcado apicalmente (a lo sumo 1/4 del largo del proceso) (Fig. 22 Mapa 3)

L. simulans simulans

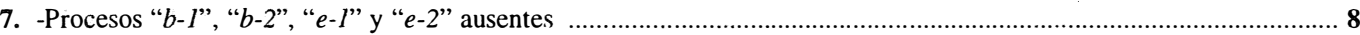

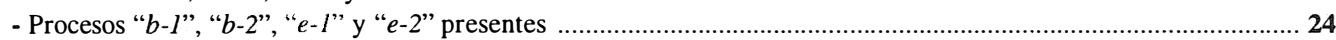

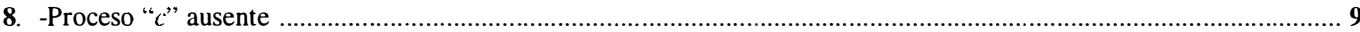

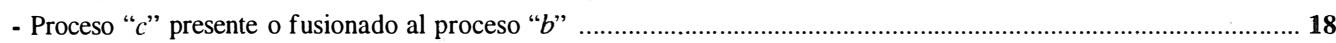

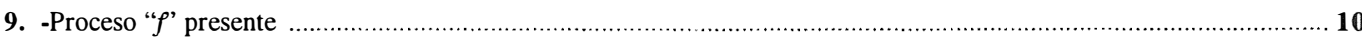

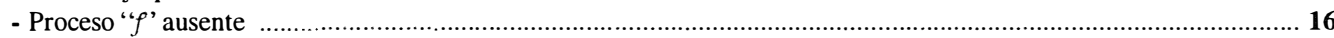

10.-Proceso " $b$ " en vista lateral, profundamente bifurcado medialmente; rama ventral más larga que la dorsal (Fig. 16, Mapa 2)

L. woldianum

- Proceso " $b$ " no bifurcado 11

11. -Proceso " $e$ " desarrollado en una fila o lóbulo dorsolateral con espinas pequeñas, fusionadas a la sección media del phallus

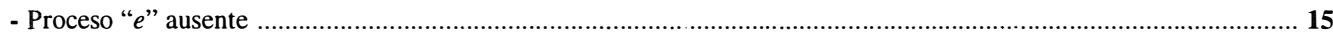

12. -Proceso " $\rho$ ' bifurcado, ápices dirigidos posteriormente (Fig. 15, Mapa 2)

L. turrialbum

- Proceso " $f$ " no bifurcado, ápice dirigido posterior o dorsalmente

13.-Proceso " $\rho$ " ovalado; proceso " $g$ " unilobulado (Fig. 11, Mapa 1)

L. salvini

- Proceso " $f$ " en vista dorsal, lenguado o redondeado; proceso " $g$ " bilobulado

14. -Proceso " $f$ " en vista dorsal, semeja una lengua, dirigido posteriormente; margen apical del proceso " $g$ " en vista ventral, con una hendidura superficial, ensanchada, no aserrada, con una proyección pequeña, puntiaguda mesalmente (Fig. 8, Mapa 1)

L. ekisi

- Proceso " $\rho$ " en vista dorsal, redondeado, corto, dirigido dorsalmente; margen apical del proceso " $g$ " en vista ventral, con una hendidura prof unda, amplia, aserrada lateralmente, en forma de "U" (Fig. 10, Mapa 1) L. rafita 
15. -Proceso " $a$ " sin proyecciones dorsales; proceso " $b$ " sinuoso, robusto, corto, alcanza la base del proceso " $f$ '; proceso " $f$ " bifurcado ligeramente, ápices dirigidos posteriormente (Fig. 9, Mapa 1)

- Proceso " $a$ " con proyecciones dorsales dirigidas anterodorsalmente; proceso " $b$ " sinuoso, delgado, largo, sobrepasa la base del proceso " $f$ '; proceso " $f$ ' bifurcado profundamente, ápices dirigidos lateralmente (Fig. 14, Mapa 2) .... L. sinuatum

16. -Proceso " $b$ " con espinas pequeñas; proceso " $e$ " dorsal, redondeado, dirigido dorsalmente; proceso " $g$ " bilobular, margen apical en vista ventral, con una hendidura prof unda, sub-acorazonada, no aserrada (Fig. 12, Mapa 1) ...... L. vitum - Proceso " $b$ " sin espinas; proceso " $e$ " dorsolateral, semeja un gancho o espina, dirigido anterodorsalmente; proceso " $g$ " unilobular, margen apical en vistal ventral, con una hendidura profunda en forma de "U"

17.-Sección media del phallus ligeramente más angosta que la apical; proceso " $e$ " robusto, conspicuo; proceso " $g$ " angosto, notablemente cóncavo dorsalmente, alargado, alcanza el margen posterior del proceso " $b$ ", ápice aserrado levemente, puntiagudo (Fig. 17, Mapa 2).

- Sección media del phallus notablemente más estrecha que la apical; proceso " $e$ " de pequeño a diminuto; proceso " $g$ " ensanchado, ligeramente cóncavo dorsalmente, alargado, usualmente sobrepasa el margen posterior del proceso " $b$ ", ápice aserrado, amplio, truncado levemente (Fig. 19, Mapa 2)

L. huismanae

18.-Proceso " $c$ " fusionado al " $b$ " en un lóbulo pareado (" $b-c$ "), apicolateral, ensanchado, seme ja una oreja, con una proyección mediolateral, curvada, puntiaguda, proyectada mesialmente; proceso " $g$ " ausente (Fig. 7, Mapa 2) ....... L. tapanti - Proceso "c" no fusionado al proceso " $b$ "

19.-Proceso " $b$ " en vista lateral, alargado, sobrepasa la base de procesos " $a$ " y " $d$ "; proceso " $d$ " subdesarrollado; proceso " $e$ " ausente; proceso " $\rho$ ' mesodorsal, bifurcado, ápices dirigidos lateralmente (Fig. 18, Mapa 2)

- Proceso " $b$ " en vista lateral, no alcanza la base de procesos " $a$ ", " $d$ "; proceso " $d$ " bien desarrollado; proceso " $e$ " presente; proceso " $f$ ' ausente

21.-Proceso " $d$ " mesodorsal; en vista lateral, reducida, aserrada apicalmente; rama basal robusta, en vista dorsal, bifurcada basalmente, sinuosa, con espinas pequeñas medianoapicalmente, en vista lateral, arqueada, ápice dirigido ventralmente (Fig. 26, Mapa 4)

L. intermedium

- Proceso " $d$ " laterodorsal; rama apical en vista lateral, alargada, pero más pequeña que la basal; esta última en vista lateral, delgada, arqueada, no bifurcada, ápice con pequeñas espinas, dirigido anteroventralmente

22.-Proceso " $e$ " lateral, en vista lateral, dirigido anteroventralmente, notablemente dividido apicalmente (Fig. 29, Mapa 4) L. furciligerum

- Proceso " $e$ " dorsolateral, fuertemente curvado basalmente, dirigido ventralmente, no dividido apicalmente

23.-Proceso " $e$ " con uno o más procesos basodorsales (Fig. 27, Mapa 4)

24.-Proceso " $d$ " con ramias apical y basal (Fig. 24, Mapa 1)

L. cheesmanae - Proceso " $d$ ” con solo la rama apical (Fig. 25, Mapa 1)

2. -Dorsal process of ejaculatory duct, as viewed laterally, subtriangular, short, not reaching dorsal margin of phallus; ventral process of ejaculatory duct, as viewed laterally, sinuous, apex barely reaching apical margin of phallus (Fig.

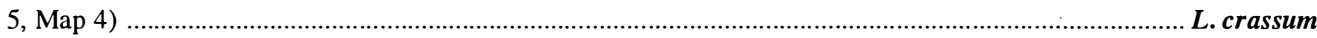
- Dorsal process of ejaculatory duct, as viewed laterally, curved slightly, elongated, reaching dorsal margin of phallus; ventral process of ejaculatory duct, as viewed laterally, curved basally, erect, fingerlike, long, extending beyond apicodorsal margin of phallus (Fig. 6, Map 4) 
3. -Process " $j$ " present

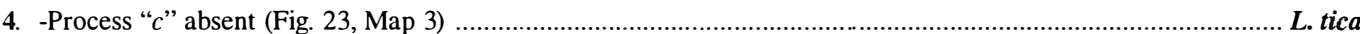

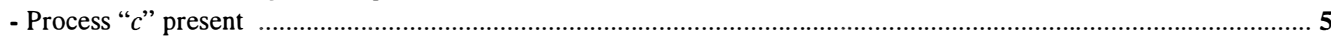

5. -Process " $b$ " short, directed posterodorsally (Fig. 21, Map 3)

L. campanum

- Process " $b$ " long, directed anteroventrally, reaching ventral margin of phallus

6

6. -Process " $b$ ", as viewed laterally, arched; process " $g$ ", as viewed laterally, curved, apex pointed; process " $j$ ", as viewed dorsally, with one or more lateral projections, deeply bifurcated apically (more than 1/3 total length of the process) (Fig. 20, Map 3)

L. asclepium

- Process " $b$ " as viewed laterally, curved rigidly; process " $g$ ", as viewed laterally, curved, apex truncate; process " $j$ ", as viewed dorsally, without lateral projections, shallowly bifurcated apically (at least 1/4 total length of the process) (Fig. 22, Map 3)

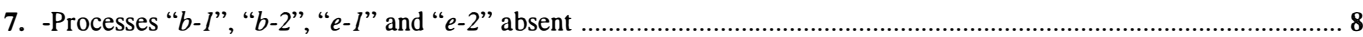

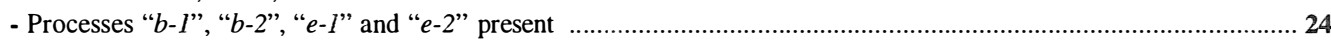

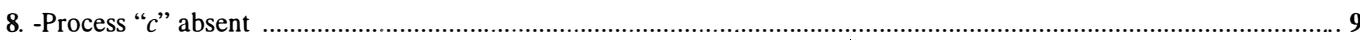

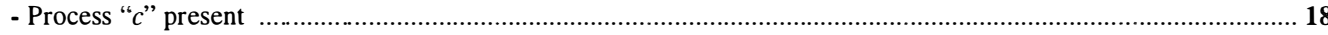

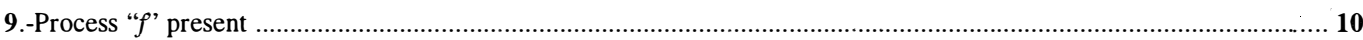

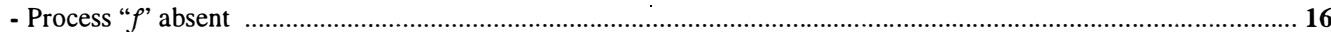

10.-Process " $b$ ", as viewed laterally, long, deeply bifurcated medially; ventral branch longer than dorsal branch (Fig. 16, Map 2)

L. woldianum

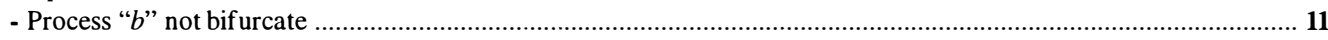

11.-Process " $e$ " developed into rows or lobes of spicules fused to midsection of phallus ........................................... 12

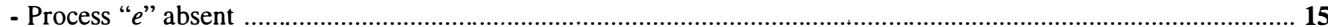

12.-Process " $\rho$ " bifurcate, apices directed posteriorly (Fig. 15, Map 2) ………............................................ L. turrialbum

- Process " $f$ " not bifurcate, apex directed posterior or dorsally

13.-Process " $f$ " oval, directed dorsally; process " $g$ " unilobed (Fig. 11, Map 1) ..................................................... L. salvini - Process " $f$ " tonguelike or rounded; process " $g$ " bilobed

14.-Process " $f$ ", as viewed dorsally, elongate, tonguelike, directed posteriorly; apical margin of process " $g$ ", as viewed ventrally, with shallow emargination, elongate, unserrated, with short, pointed, mesal projection (Fig. 8, Map 1) .. L. ekisi - Process " $f$ ", as viewed dorsally, rounded, short, directed dorsally; apical margin of process " $g$ ", as viewed ventrally, with deep, wide, serrated laterally, U-shaped emargination (Fig. 10, Map 1)

L. rafita

15. -Process " $a$ " without dorsal projections; process " $b$ " sinuous, stout, short, reaching base of process " $f$ "; process " $\rho$ " bifurcated slightly, apices directed posteriorly (Fig. 9, Map 1)

L. fortunum

- Process " $a$ " with dorsal projections directed anterodorsally; process " $b$ " sinuous, slender, elongate, extending beyond base of process " $f$ '; process " $f$ ' bifurcate, apices directed laterally (Fig. 14, Map 2)

L. sinuatum

16. -Process " $b$ " with spicules; process " $e$ " dorsal, rounded, directed dorsally; process " $g$ " bilobed, apical margin, as viewed ventrally, with deep, unserrated, subheartlike emargination (Fig. 12, Map 1) ......................................... L. vitum - Process " $b$ " without spicules; process " $e$ " dorsolateral, hooklike or spinelike, directed anterodorsally; process " $g$ " unilobed, apical margin as viewed ventrally with deep, U-shaped emargination

17.-Midsection of phallus slightly narrower than apical section; process " $e$ " stout, conspicuous; process " $g$ " narrow, distinctly concave dorsally, elongate, barely reaching posterior margin of process " $b$ ", apex slightly serrated, pointed (Fig. 17, Map 2) L. flintorum - Midsection of phallus distinctly narrower than apical section; process " $e$ " short to minute; process " $g$ " enlarged, slightly concave middorsally, elongate, usually extending beyond posterior margin of process " $b$ ", apex serrated, wide, slightly truncated (Fig. 19, Map 2) 
18.-Process " $c$ " fused to " $b$ " into apicolateral in flated, paired lobes (" $b-c$ "), with slender, curved, midlateral projection with pointed apex, directed mesally; process " $g$ " absent (Fig. 7, Map 2) L. tapanti - Process " $c$ " not fuse to process " $b$ "

19.-Process " $b$ " elongate, extending beyond base of process " $a$; and "d"; process " $d$ " subdeveloped; process " $e$ " absent; process " $f$ " dorsomesal, bifurcate, apices directed laterally (Fig. 18, Map 2) ................................................. L. hamuli - Process " $b$ " short, not reaching base of processes " $a$ ", " $d$ "; process " $d$ " well developed; process " $f$ " absent ........... 20

20.-Process " $d$ ", as viewed laterally, unibranched, strongly curved basally, directed ventrally (Fig. 13, Map 3) .. L. albovirens - Process " $d$ " bibranched, asymmetric; basal branch longer than apical branch

21.-Process " $d$ " dorsomesal, apical branch reduced, serrated apically; basal branch of process " $d$ ", as viewed dorsally, stout, bifurcated basally, sinuous, with small spines apicomiddly, as viewed laterally, arched, apex directed ventrally (Fig. 26, Map 4) L. intermedium

- Process " $d$ " dorsolateral, apical branch elongate, shorter than basal branch; basal branch of process " $d$ ", as viewed laterally, slender, arched, not bifurcate, apex with small spines, directed anteroventrally

22. -Process " $e$ " lateral, as viewed laterally, directed anteroventrally, deeply divided apically (Fig. 29, Map 4) ... L. furciligerum

- Process " $e$ " dorsolateral, rigidly curved basally, directed ventrally, not divided apically

23.-Process " $e$ " with one or more basodorsal processes (Fig. 27, Map 4)

L. complexum

- Process " $e$ " without basodorsal processes (Fig. 28, Map 4)

L. forficulum

24. - Process " $d$ " with apical and basal branches (Fig. 24, Map 1)

L. cheesmanae - Process " $d$ " only with apical branch (Fig. 25, Map 1)

\section{AGRADECIMIENTOS}

Se agradece los valiosos comentarios y sugerencias de Oliver S. Flint, Jr., Ralph W. Holzenthal, Atilano Contreras Ramos, Roger J. Blahnik y de los revisores anónimos. Agradecimientos a O. S. Flint, Jr. por el préstamo de especímenes del Museo Nacional de Historia Natural (NMHN, "Smithsonian Institution"), a Krista Kurda por las ilustraciones de la Fig. 4, a Julie J. Martínez por las ilustraciones de la Fig. 1 , y a Ingrid Gibson por su colaboración bibliográfica. Reconocimiento y profunda gratitud a Ralph W. Holzenthal por su guía, apoyo constante, y quien concibió el Proyecto "Inventario de los Trichoptera de Costa Rica", el presente manuscrito es parte de los resultados de este Proyecto apoyado por la "U.S. National Science Foundation" (USNSF, donaciones BSR8917684 y DEB-9400632). A Gloria Stella Santacruz M. por su valiosa colaboración y apoyo. Finalmente, a todos los amigos biólogos, ecólogos y limnólogos en Latinoamérica que han presionado y ayudado para ver publicada esta investigación. Este escrito es el número 98117-0018 de la serie de publicaciones "Scientific Journal Series" de la Estación Experimental de Agricultura de Minnesota, St. Paul, Minnesota, E.U.A.

\section{RESUMEN}

El género Leptonema Guérin 1843 (Hydropsychidae: Macronematinae) es uno de los géneros de Trichoptera más abundantes y más ampliamente distribuidos en el Nuevo Mundo. En Costa Rica son conocidas 24 especies. El presente traba jo incluye la descripción e ilustración del genital del macho de una especie no descrita para Costa Rica: Leptonema tica. Además de la descripción diagnóstica y ilustración de la larva, se incorporan las descripciones diagnósticas e ilustraciones del phallus de los machos de las otras 24 especies. Anotaciones de la historia natural del adulto y la larva del género son incluidas. Finalmente, se incluyen una clave de identificación en inglés y español de las especies conocidas de Leptonema para Costa Rica y se ilustran sus registros de distribución en Costa Rica.

\section{REFERENCIAS}

Aguila S., Y. 1992. Systematic catalogue of the caddisflies of Panama (Trichoptera), p. 532-548. In D. Quintero 
\& A. Aiello (eds.). Insects of Panama and Mesoamerica: Selected studies. Oxford Univ., Oxford.

Angrisano, E. B. 1995. Insecta Trichoptera, p. 1199-1237, Tomo III. In E.C. Lopretto \& G. Tell (dirs.). Ecosistemas de aguas continentales: Metodologías para su estudio. Ed. Sur, Argentina.

Banks, N. 1913. Neuropteroid insects from Brazil. Psyche 20: 83-89.

Banks, N. 1914. Neuroptera and Trichoptera from Costa Rica. Entomol. News 25: 149-150.

Betten, C. \& M.E. Mosley. 1940. The Francis Walker types of Trichoptera in the British Museum. Brit. Mus. (Nat. Hist.). 248p.

Bueno-Soria, J. \& O.S. Flint, Jr. 1978. Catálogo sistemático de los tricópteros de México (Insecta: Trichoptera), con algunos registros de Norte, Centro y Sudamérica. An. Inst. Biol. Univ. Autón. México 49, Ser. Zool. (1): 189-218.

Correa G., M.M. 1988. Trichoptera, p. 145-168. In G. Roldán Pérez (ed.). Guía para el estudio de macroinvertebrados acuáticos del Departamento de Antioquia. Colciencias. Antioquia, Colombia.

Correa, M., T. Machado \& G. Roldán. 1981. Taxonomía y ecología del orden Trichoptera en el Departamento de Antioquia en diferentes pisos altitudinales. Actual. Biol. 10 (36): 35-48.

Fischer, F.C.J. 1947. De Leptonema soorten van het Leidsch Museum (Trichoptera). Tijdschr. Entomol. 88: 313-315.

Fischer, F.C.J. 1963. Trichopterum Catalogus IV. Hydropsychidae, Arctopsychidae. Ned. Entomol. Veren., Amsterdam. 223p.

Fischer, F.C. 1972. Trichopterum Catalogus XIII. Supplemento de Volúmenes III y IV. Ned. Entomol. Veren., Amsterdam. 172p.

Flint, O.S., Jr. 1964. The caddisflies (Trichoptera) of Puerto Rico. Univ. Puerto Rico, Agr. Exp. Sta, Tech. Pap. 40: 1-80.

Flint, O.S., Jr. 1966. Studies of Neotropical caddisflies, III: Types of some species described by Ulmer and Brauer. Proc. U. S. Nat. Mus. 120 (3559): 1-20.

Flint, O.S., Jr. 1967. Studies of Neotropical caddisflies, V: Types of the species described by Banks and Hagen. Proc. U. S. Nat. Mus. 123 (3619): 1-37.

Filint, O.S., Jr. 1968. Bredin-Archbold-Smithsonian biological survey of Dominica, 9. The Trichoptera (cad- disflies) of the Lesser Antilles. Proc. U. S. Nat. Mus. 125 (3665): 1:86.

Flint, O.S., Jr. 1972. Studies of Neotropical caddisflies, XIV: On a collection from northern Argentina. Proc. Biol. Soc. Wash. 85: 223-248.

Flint, O.S., Jr. 1974a. Studies of Neotropical caddisflies, XV: The Trichoptera of Suriname. Stud. Fauna Suriname other Guyanas 14: 1-151.

Flint, O.S., Jr. 1974b. Studies of Neotropical caddisflies, XVII: The genus Smicridea from North and Central America (Trichoptera: Hydropsychidae). Smithson. Contrib. Zool. 167: 1-65.

Flint, O.S., Jr. 1978. Studies of Neotropical caddisflies, XXII: Hydropsychidae of the Amazon Basin (Trichoptera). Amazonia 6: 373-421.

Flint, O.S., Jr. 1981. Studies of Neotropical caddisflies, XXVIII: The Trichoptera of the Río Limon Basin, Venezuela. Smithson. Contrib. Zool. 330: 1-61.

Flint, O.S., Jr. 1989. Studies of Neotropical caddisflies, XXXIX: The genus Smicridea in the Chilean Subregion (Trichoptera: Hydropsychidae). Smithson. Contrib. Zool. 472: 1-45.

Flint, O.S., Jr. 1991. Studies of Neotropical caddisflies, XLV: The taxonomy, phenology, and faunistics of the Trichoptera of Antioquia, Colombia. Smithson. Contrib. Zool. 520: 1-113.

Flint, O.S., Jr. \& J.B. Wallace. 1980. Studies of Neotropical caddisflies, XXV: The inmature stages of Blepharopus diaphanus and Leptonema columbianum (Trichoptera: Hydropsychidae). Proc. Biol. Soc. Wash. 93: 178-193.

Flint, O.S., Jr. \& J. Bueno-Soria. 1987. Studies of Neotropical caddisflies, XXXVII: The genus Calosopsyche in Central America, with descriptions of its inmature stages (Trichoptera: Hydropsychidae), p. 29-37. In M. Bournaud \& H. Tachet (eds.). Proc. 5th Int. Symp. Trichoptera. Junk, La Haya.

Flint, O.S., Jr., J.F. McAlpine \& H.H. Ross. 1987. A revision of the genus Leptonema (Trichoptera: Hydropsychidae: Macronematinae). Smithson. Contrib. Zool. 450: 1-193.

Greiff, M. 1985. Spanish-English-Spanish. Lexicon of entomological and related terms. $\mathrm{CAB}$, Comm. Inst. Entomol., Londres. 102 p. Index I, 1-115 p. Index II, $1-43 \mathrm{p}$.

Guérin-Meneville, M.F.E. 1843. Insecta: Trichoptera, 3b, p. 395-397. In Cuvier. Iconographie du Régne Animal. Paris. 
Harrison, A.D. \& J.J. Rankin. 1976. Hydrobiological studies of eastern Lesser Antillean Islands, II, St. Vincent: Freshwater fauna-its distribution, tropical river zonation and biogeography. Arch. Hydrobiol., Suppl. 50: 275-311.

Holzenthal, R.W. 1988. Catálogo sistemático de los tricópteros de Costa Rica (Insecta: Trichoptera). Brenesia 29: 51-82.

Marlier, G. 1962. Gènèra des trichoptères de l'Afrique. Ann. Mus. Roy. Afri. Cent. Sci. Zool., Série 8vo, 109: 1-263.

McCafferty, W.P. 1983. Caddisflies (Order Trichoptera), p. 237-273. In W.P. McCaferty. Aquatic Entomology. 3ra. ed. Jones \& Bartlett, Boston.

McElravy, E.P., V.H. Resh, H. Wolda \& O.S. Flint, Jr. 1981. Diversity of adult Trichoptera in a "non-seasonal" tropical environmental, p. 149-156. In G.P. Moretti (ed.). Proc. 3rd. Int. Symp. Trichoptera. Junk, La Haya.

McElravy, E.P., H. Wolda \& V.H. Resh. 1982. Seasonality and annual variability of caddisfly adults (Trichoptera) in a "non-seasonal" tropical environmental. Arch. Hydrobiol. 94: 302-317.

Morse, J.C. \& R.W. Holzenthal. 1996. Trichoptera Genera, p. 350-386. In R.W. Merritt \& K.W. Cummins (eds.). An introduction to the aquatic insects of North America. 3ra. ed. Kendall/Hunt Publ. Co., Dubuque, Iowa.

Mosely, M.E. 1933. A revision of the genus Leptonema. Brit. Mus. (Nat. Hist.), Londres. 69 p.

Mosely, M.E. 1939. Leptonema pallidum Guérin (Trichoptera). Ann. Mag. Nat. Hist. Ser. 11, 4: 310-314.

Muñoz-Quesada, F. 1997. Five new species and a record of Costa Rican Leptonema (Trichoptera: Hydropsychidae). Proc. Entomol. Soc. Wash. 99: 115-132.

Navás, L. 1927. Neuropteren, Megalopteren, Plecopteren und Trichopteren aus dem Deutsch. Entomol. Inst. (Berlin-Darhlem). Entomol. Mitt. 16: 37-43.

Navás, L. 1933. Insectos Suramericanos. Séptima Ser. Rev. Acad. Cien. Madrid 30: 303-314.

Neboiss, A. \& J.C. Dean. 1991. Trichoptera, p. 787-816, Volumen II. In CSIRO (ed.). The insects of Australia. 2da. ed. Cornell Univ., Ithaca, Nueva York.

Quintanilla, R.H. \& C.P. Fraga. 1969. Glosario de términos entomológicos. EUDEBA, Buenos Aires. 106 p.

Roback, S.S. 1966. The Catherwood Foundation PeruvianAmazon Expedition: Limnological and Systematic
Studies. XI - The Trichoptera Larvae and Pupae. Monographs of the Academy of Natural Sciences of Philadel phia, 14:235-303.

Ross, H.H. 1982. Introducción a la entomología general y aplicada. 5ta. ed. Ed. Omega, Barcelona. 536 p.

Schmid, F. 1964. Contribution a l'Étude des Trichoptéres Neotropicaux, V. Tijdschr. Entomol. 107: 307-339.

Scott, K.M.F. 1983. On the Hydropsychidae (Trichoptera) of southern Africa with keys to African genera of imagos, larvae and pupae and species lists. Ann. Cape Prov. Mus. (Nat. Hist.). 14: 299-422.

Snodgrass, R.E. 1993. Principles of insect morphology. Cornell Univ., Ithaca, Nueva York. 667p.

Torre-Bueno, J.R. de la. 1989. The Torre-Bueno Glossary of Entomology. New York Entomol. Soc., Nueva York. 840 p.

Ulmer, G. 1905. Zur Kenntniss aussereuropäischer Trichopteren. Stett. Entomol. Ziet. 66: 1-119.

Ulmer, G. 1907a. Monographie der Macronematinae, Trichoptera, Fasc. 6 (2): p. 1-121, In Collections Zoologiques du Baron Edm. de Selys Longchamps. Bruselas.

Ulmer, G. 1907b. Trichoptera, 60: p. 1-259. In P. Wytsman (ed.). Genera Insectorum. Bruselas.

Ulmer, G. 1957. Köcherfliegen (Trichopteren) von den Suinda-Insel (Teil 3). Arch. Hydrobiol., Suppl. 23: 109-470.

Walker, F. 1852. Catalogue of the neuropterous insects in the collection of the British Museum, Part I: Phryganides-Perlides. Londres. 192 p.

Weidner, H. 1964. Die Entomologischen Sammlungen des Zoologischen Staatsinstituts und Zoologischen Museums Hamburg. Mitt. Hamburg. Zool. Mus. Inst. 62: 55-100.

Wiggins, G.B. 1996a. Larvae of the North American Caddisfly Genera (Trichoptera). 2da. ed. Univ. Toronto, Toronto. 457p.

Wiggins, G.B. 1996b. Trichoptera Families, p. 309-349. In R.W. Merritt \& K.W. Cummins (eds.). An introduction to the aquatic insects of North America. 3ra. ed. Kendall/Hunt Publ. Co., Dubuque, Iowa.

Williams, N.E. \& G.B. Wiggins. 1981. A proposed setal nomenclature and homology for larval Trichoptera, p. 421-429. In G.P. Moretti (ed.). Proc. 3rd. Int. Symp. Trichoptera. Junk, La Haya. 